\title{
NEW FORTRAN COMPUTER PROGRAMS TO ACQUIRE AND PROCESS ISOTOPIC MASS SPECTROMETRIC DATA
}

Operator's Manual

D. H. Smith

H. S. McKown

Analytical Chemistry Division

Date Published: September 1993

NOTICE This document contains information of a preliminary nature. It is subject to revision or correction and therefore does not represent a final repore.

OAK RIDGE NATIONAL LABORATORY

Oak Ridge, Tennessee 37831

managed by

MAPTIN MARIETTA ENERGY SYSTEMS, INC.

for the

U.S. DEPARTMENT OF ENERGY

under Contract No. DE-AC05-84OR21400 
1. INTRODUCTION $\ldots \ldots \ldots \ldots \ldots \ldots \ldots \ldots \ldots \ldots \ldots \ldots \ldots \ldots \ldots \ldots$

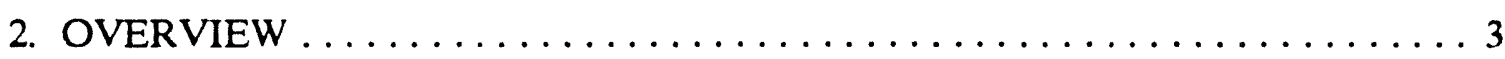

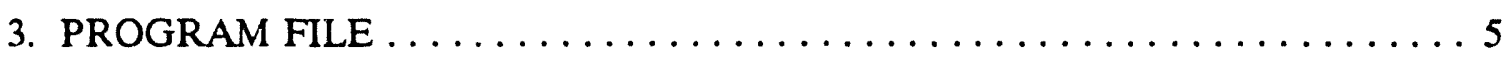

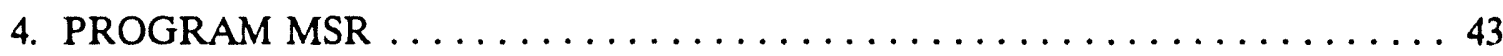

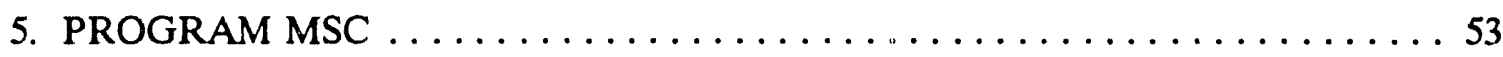

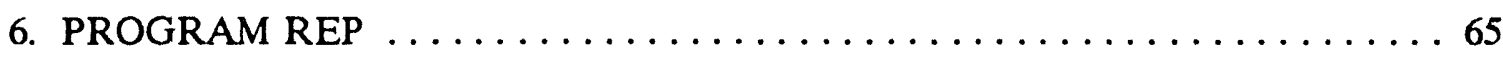

7. PROGRAMS TRAN AND REC $\ldots \ldots \ldots \ldots \ldots \ldots \ldots \ldots \ldots \ldots \ldots \ldots \ldots \ldots$

8. MISCELLANEOUS PROGRAMS $\ldots \ldots \ldots \ldots \ldots \ldots \ldots \ldots \ldots \ldots \ldots$

9. COMPUTER CONSIDERATIONS $\ldots \ldots \ldots \ldots \ldots \ldots \ldots \ldots \ldots \ldots$

10. OPERATING A MASS SPECTROMETER $\ldots \ldots \ldots \ldots \ldots \ldots \ldots \ldots \ldots$

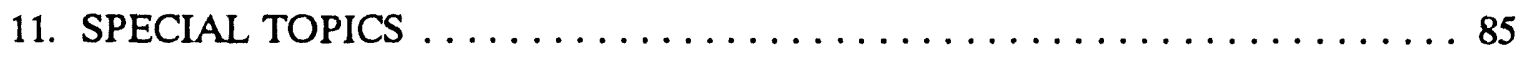

12. QUALITY ASSURANCE AND CONTROL $\ldots \ldots \ldots \ldots \ldots \ldots \ldots$

13. APPENDIX: EXAMPLES OF INPUT $\ldots \ldots \ldots \ldots \ldots \ldots \ldots \ldots \ldots$ 
TABLE OF CONTENTS

1. INTRODUCTION $\ldots \ldots \ldots \ldots \ldots \ldots \ldots \ldots \ldots \ldots \ldots \ldots \ldots \ldots \ldots \ldots$

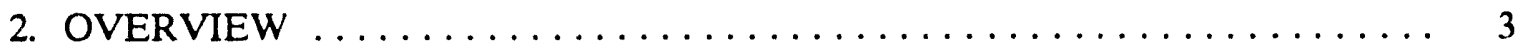

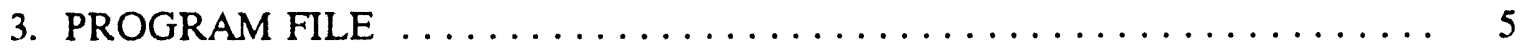

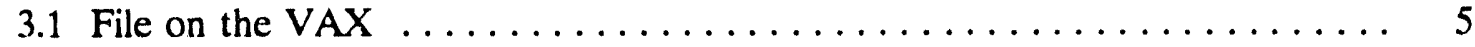

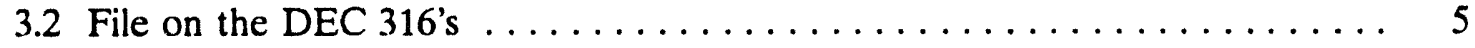

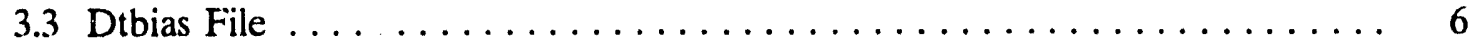

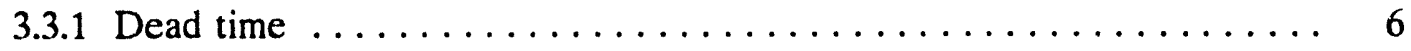

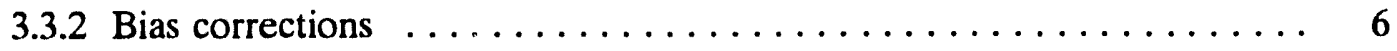

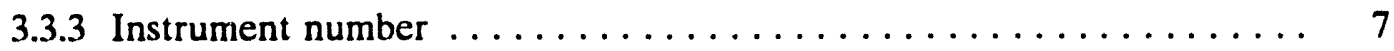

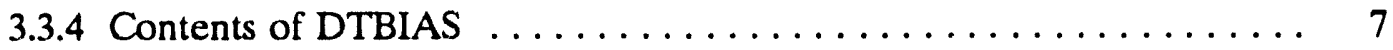

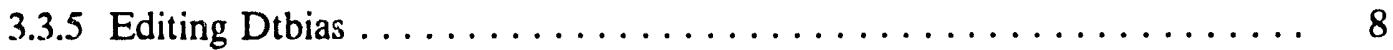

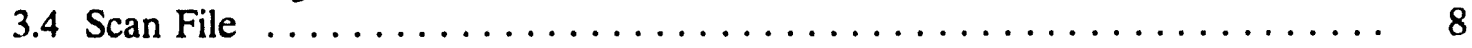

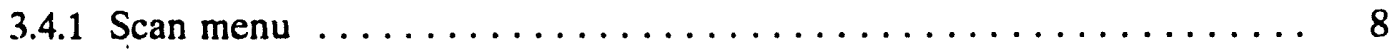

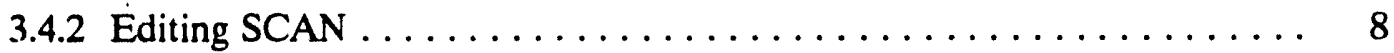

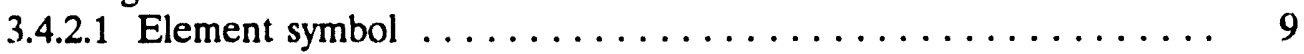

3.4.2.2 Highest subgroup number $\ldots \ldots \ldots \ldots \ldots \ldots \ldots \ldots \ldots \ldots \ldots$

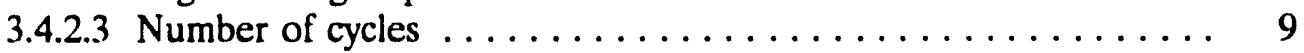

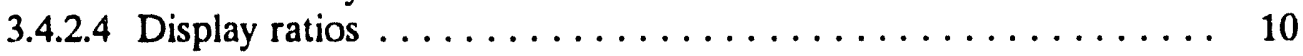

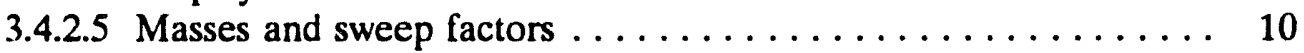

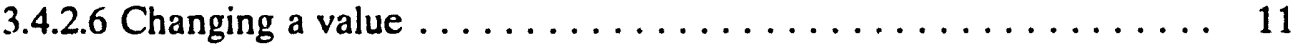

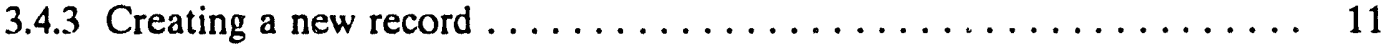

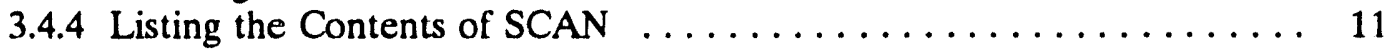

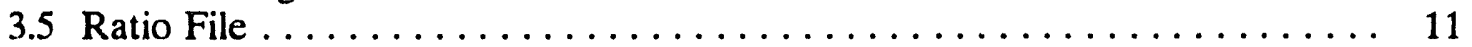

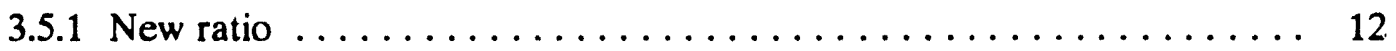

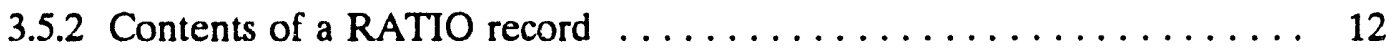

3.5.2.1 Element symbol and record number $\ldots \ldots \ldots \ldots \ldots \ldots \ldots \ldots \ldots$

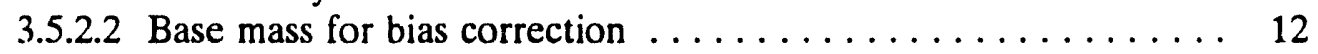

3.5.2.3 Normalizing mass for bias correction $\ldots \ldots \ldots \ldots \ldots \ldots \ldots$

3.5.2.4 Mass to be subtracted from all other SGs . . . . . . . . . 13

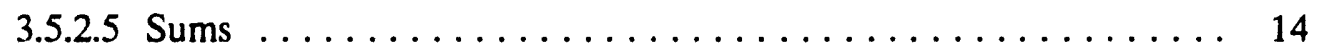

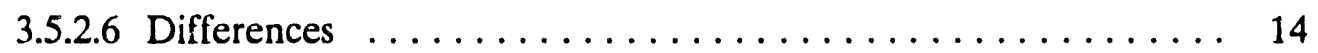

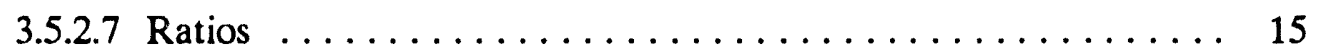

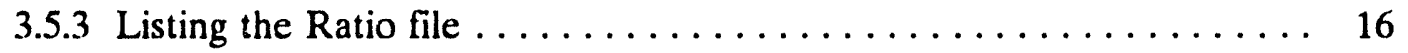

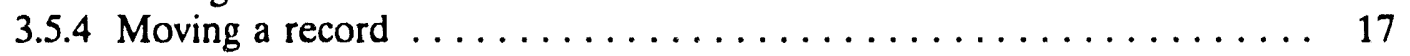

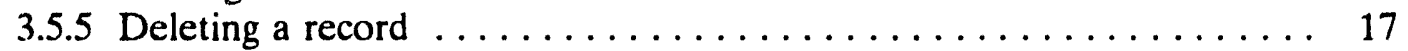

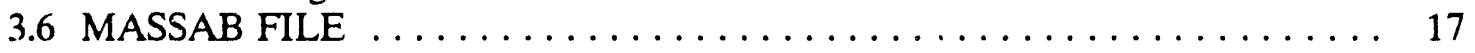

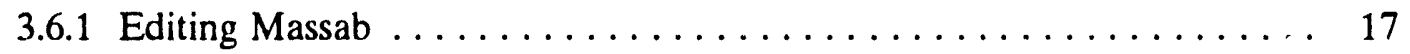

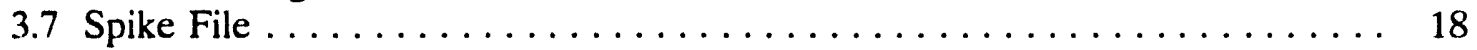

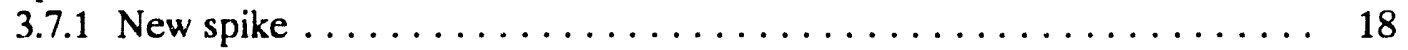

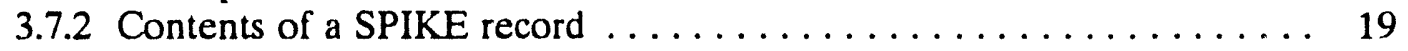

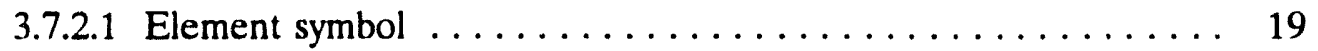

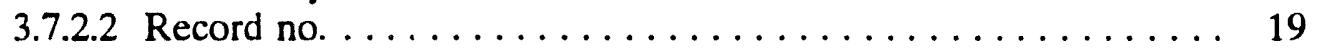

3.7.2.3 Label for spike . . . . . . . . . . . . . . . 19 


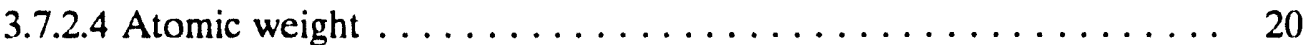

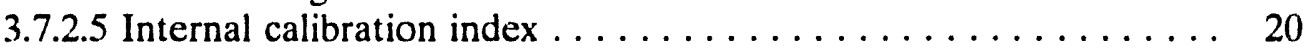

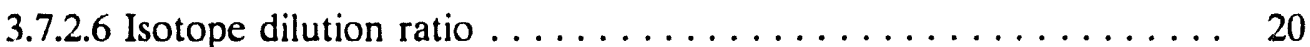

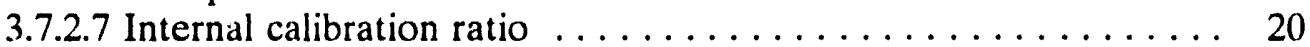

3.7.2.8 Masses and abundances .................... 21

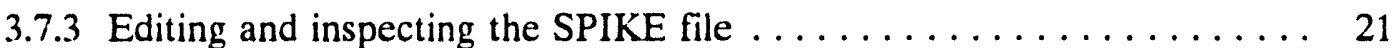

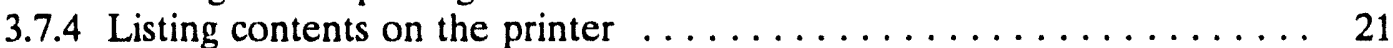

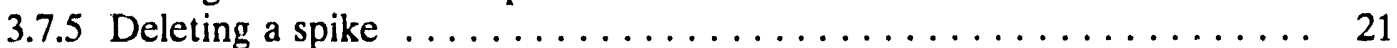

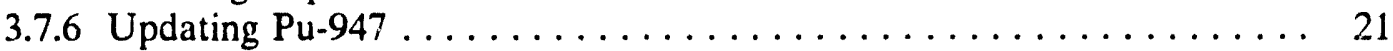

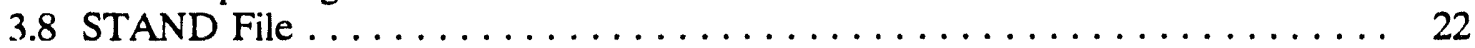

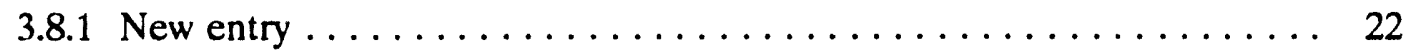

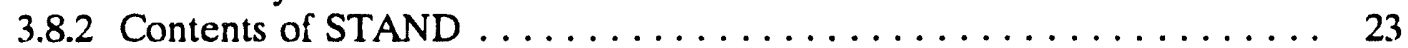

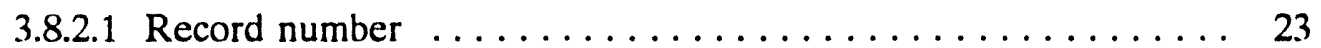

3.8 .2 .2 Element . . . . . . . . . . . . . . . . . . 23

3.8 .2 .3 Label . . . . . . . . . . . . . . . . . . . 23

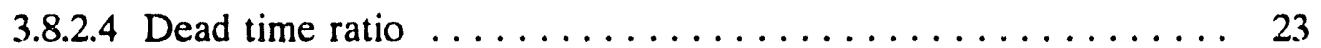

3.8 .2 .5 Bias ratio . . . . . . . . . . . . . . . . . . 24

3.8 .2 .6 Ratios ... . . . . . . . . . . . . . . . . . . . 24

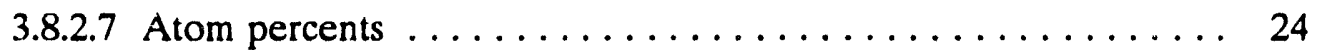

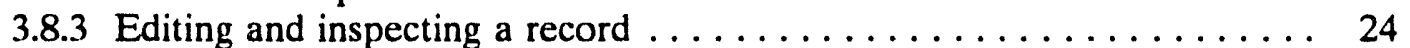

3.8 .4 Listing labels . . . . . . . . . . . . . . . . . . . . . . . 24

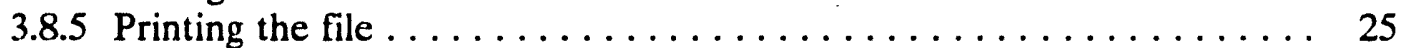

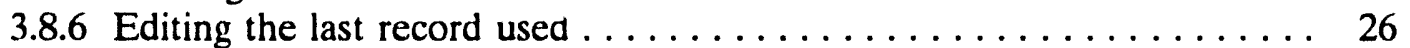

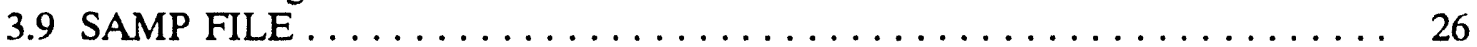

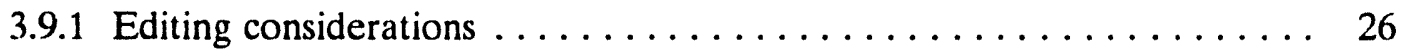

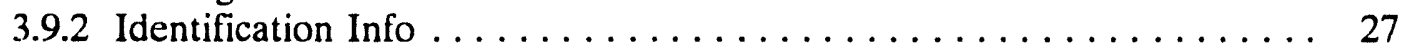

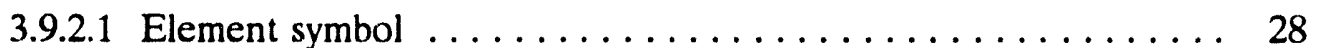

3.9 .2 Title . . . . . . . . . . . . . . . . . . 28

3.9.2.3 Unique sample id code . . . . . . . . . . . . . . . . 28

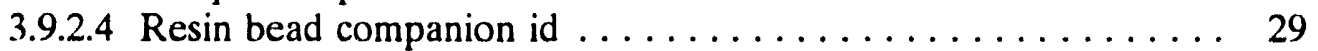

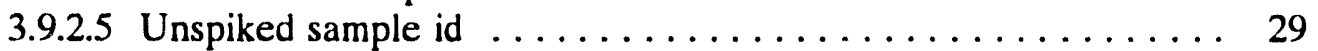

3.9.2.6 Blank companion id ...................... 29

3.9.2.7 Contaminant elements . . . . . . . . . . . . . . . . . . . 29

3.9.2.8 Batch code . . . . . . . . . . . . . . . . . . . . . . . . . . 29

3.9.2.9 Analysis date . . . . . . . . . . . . . . . . . 29

3.9.2.10 Date to which to correct $\mathrm{Pu}$ isotopic $\ldots \ldots \ldots \ldots \ldots \ldots$

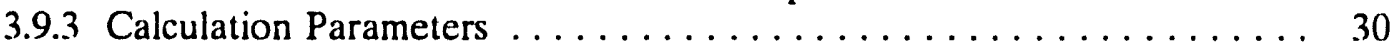

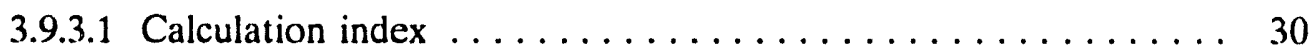

3.9.3.2 Mixture index ...................... 30

3.9.3.3 No. of passes through calculations $\ldots \ldots \ldots \ldots \ldots \ldots \ldots \ldots$

3.9.3.4 Blank index ....................... 31

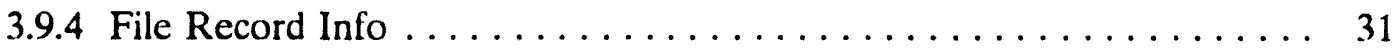

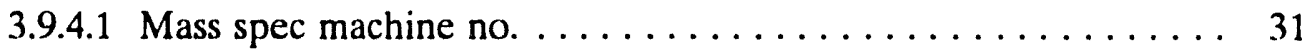

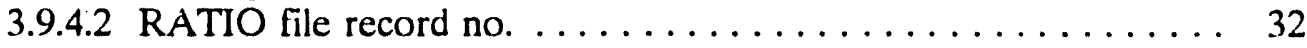

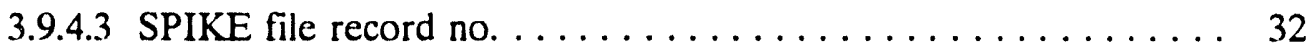

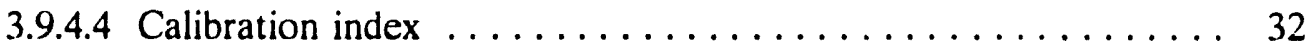

3.9.4.5 STAND file record no. for calibration $\ldots \ldots \ldots \ldots \ldots \ldots \ldots . \ldots \ldots$ 


\section{vii}

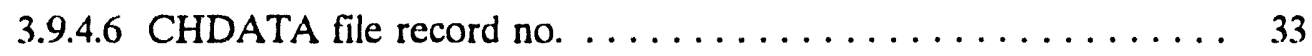

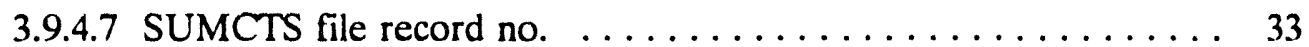

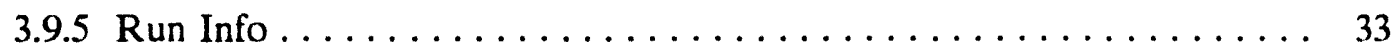

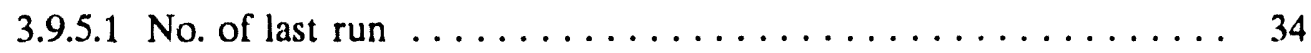

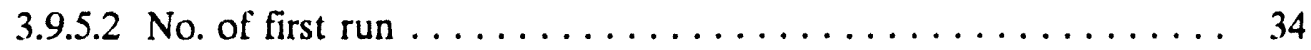

3.9.5.3 No. of the highest subgroup $\ldots \ldots \ldots \ldots \ldots \ldots \ldots \ldots \ldots \ldots \ldots$

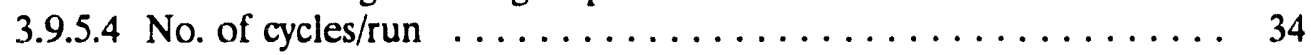

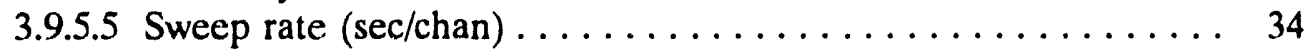

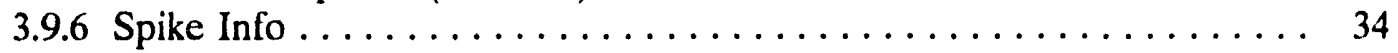

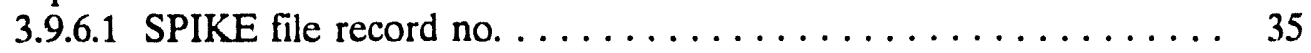

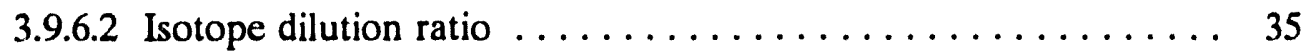

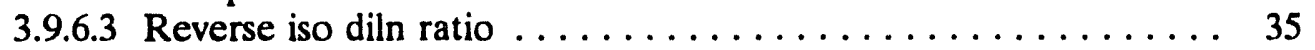

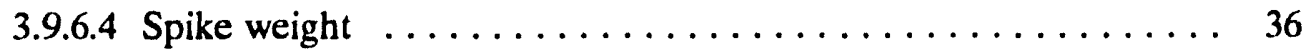

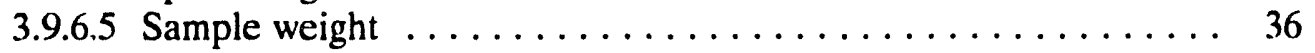

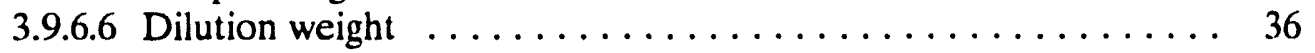

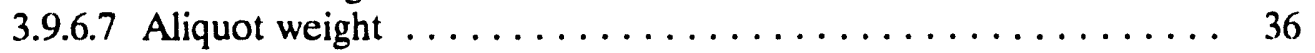

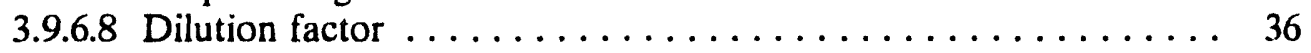

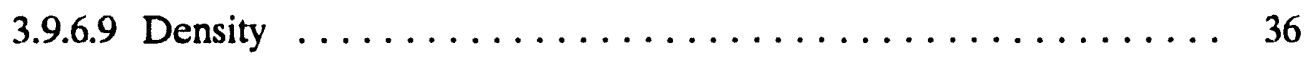

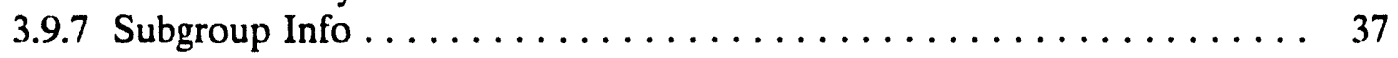

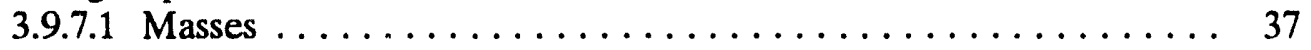

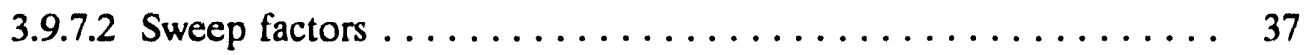

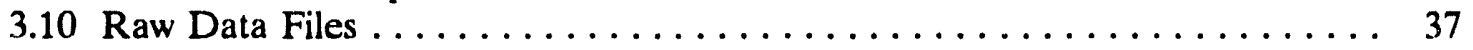

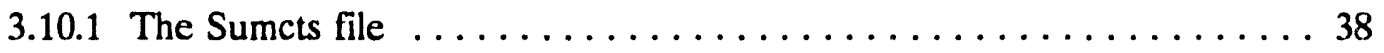

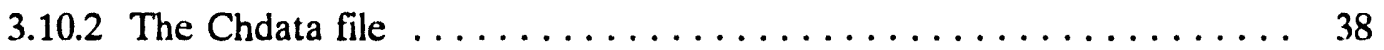

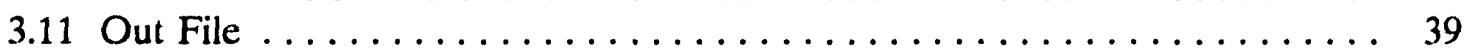

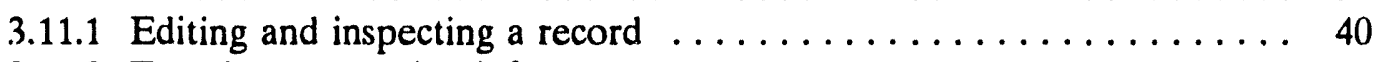

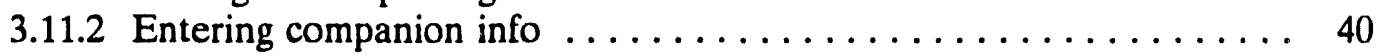

3.11.3 Entering PERMANENT companion info . . . . . . . . . . 40

3.11 .4 Listing permanent companions .................. 41

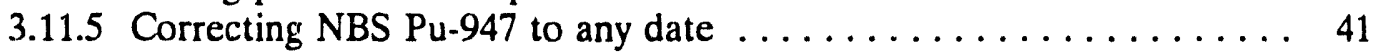

3.11.6 Changing the last record in Out $\ldots \ldots \ldots \ldots \ldots \ldots \ldots \ldots \ldots \ldots \ldots$

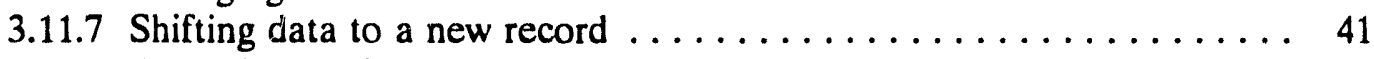

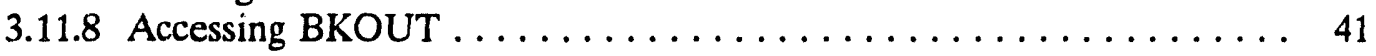

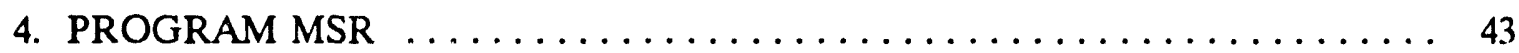

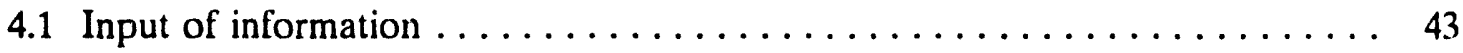

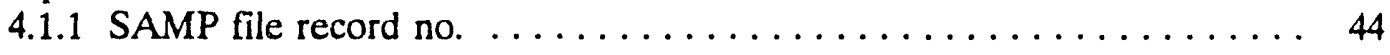

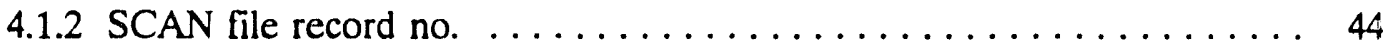

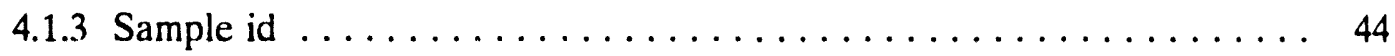

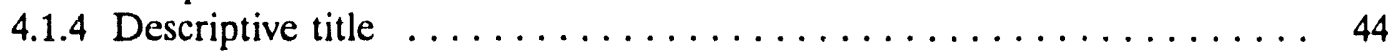

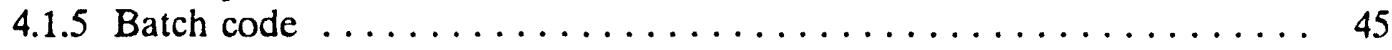

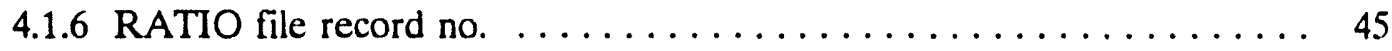

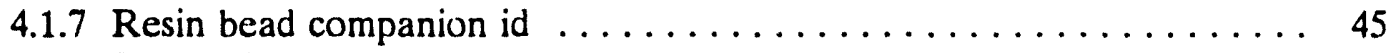

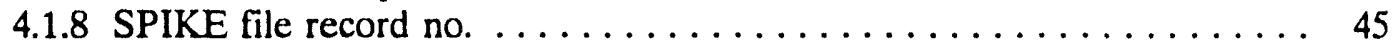

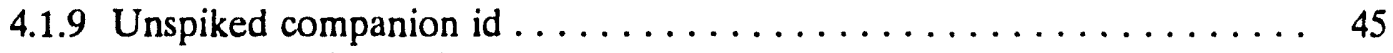

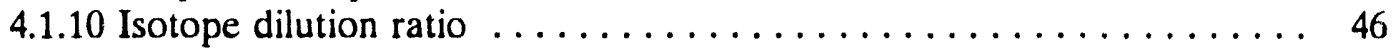

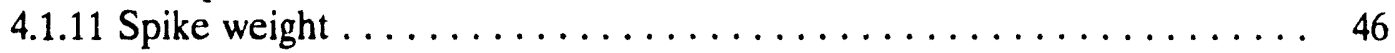

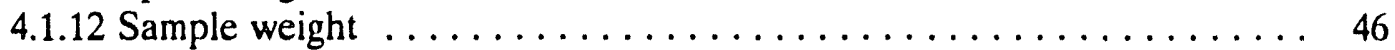


viii

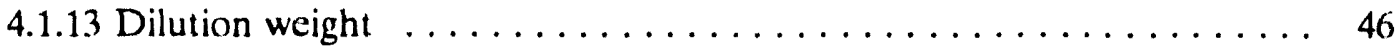

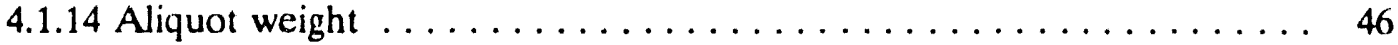

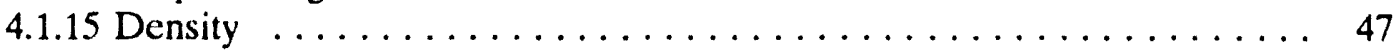

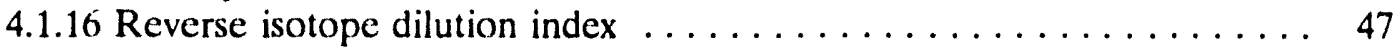

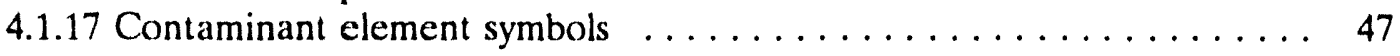

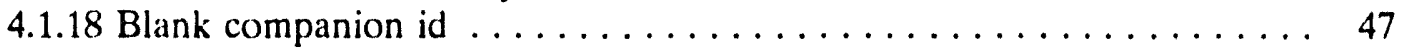

4.1 .19 Index no. to correct, 0 to proceed . . . . . . . . . . . 47

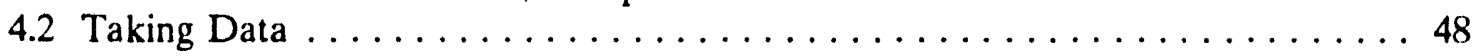

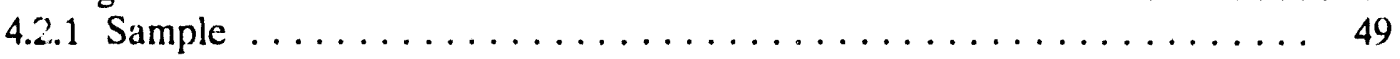

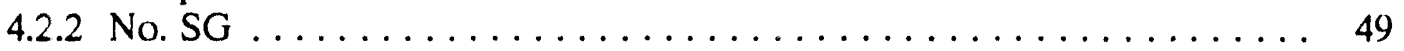

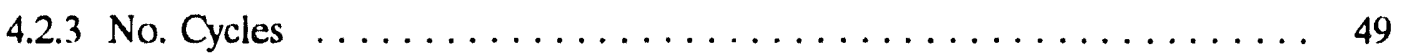

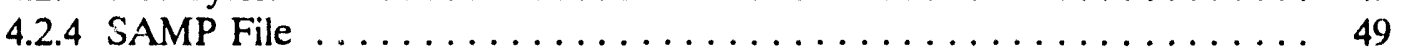

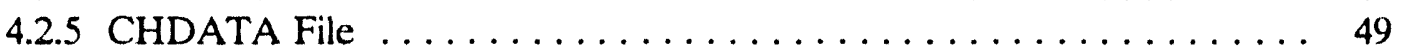

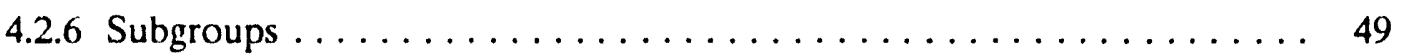

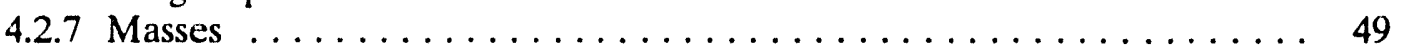

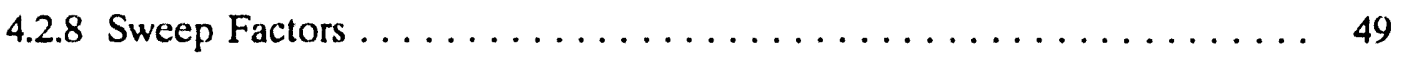

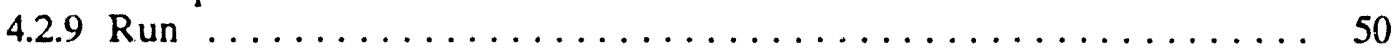

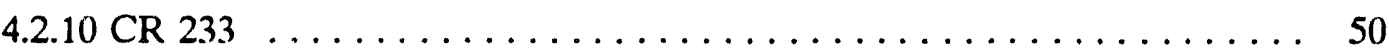

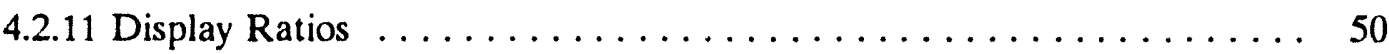

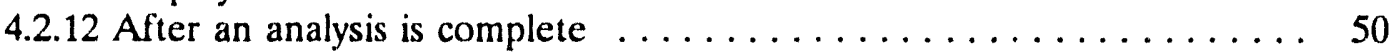

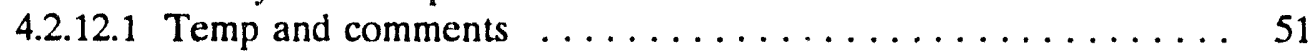

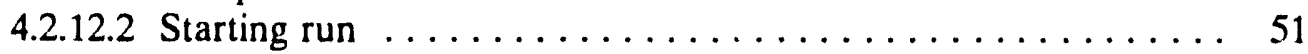

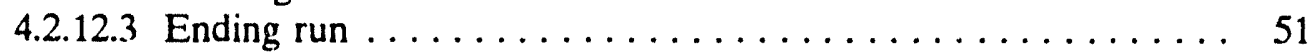

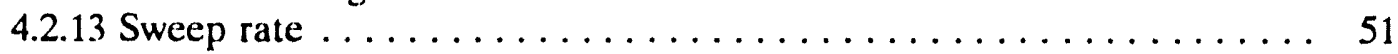

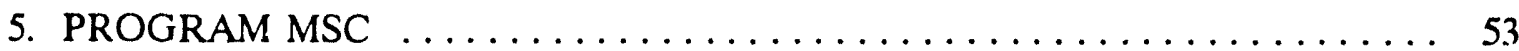

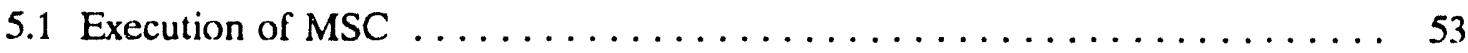

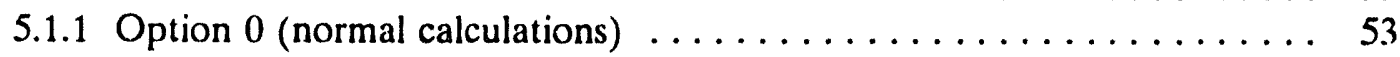

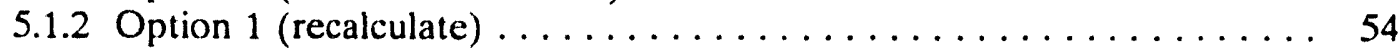

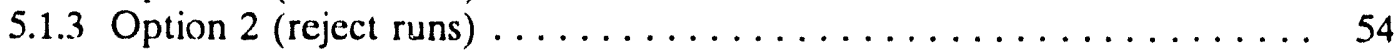

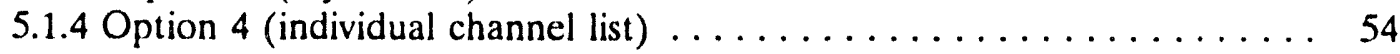

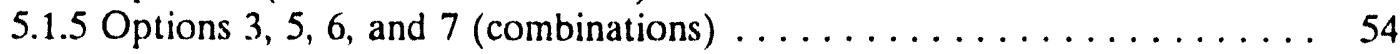

5.1.6 Option 9 (list SAMP.DAT contents) $\ldots \ldots \ldots \ldots \ldots \ldots \ldots \ldots$

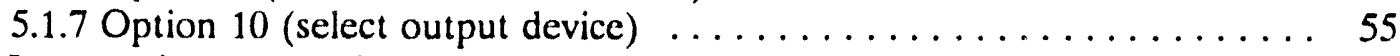

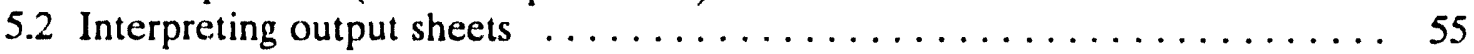

5.2 .1 First output sheet: Raw data $\ldots \ldots \ldots \ldots \ldots \ldots \ldots \ldots \ldots \ldots$

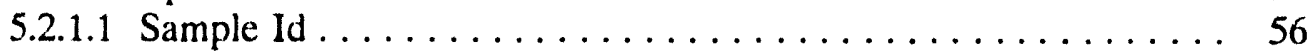

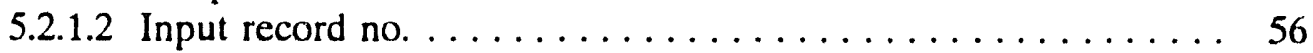

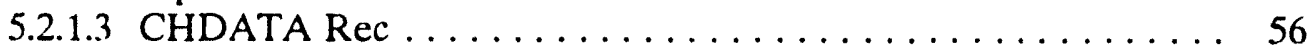

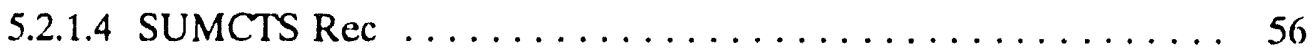

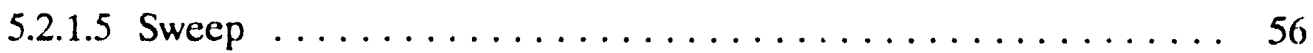

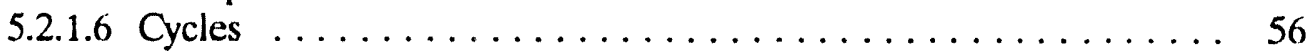

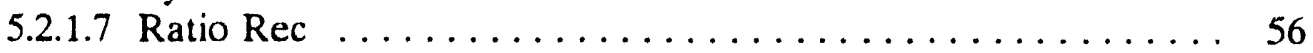

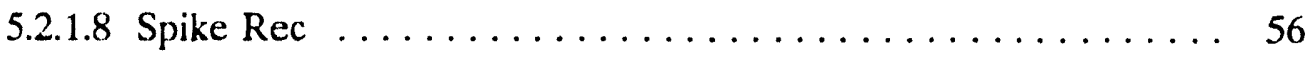

5.2 .1 .9 Corrected channels ..................... 56

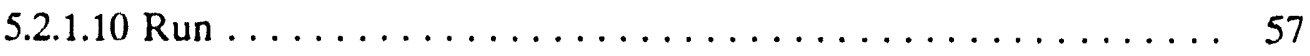

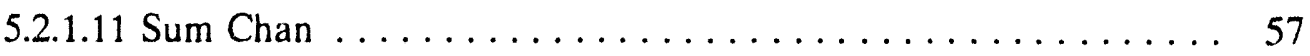

5.2 .1 .12 Raw Counts . . . . . . . . . . . . . . . . 57 


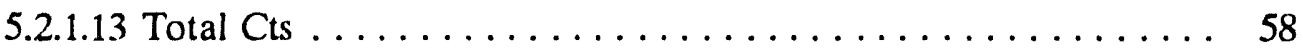

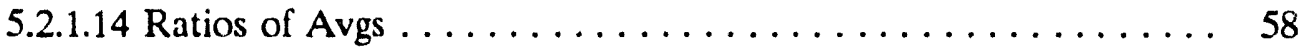

5.2 .2 Second output sheet: Ratios and averages ............. 58

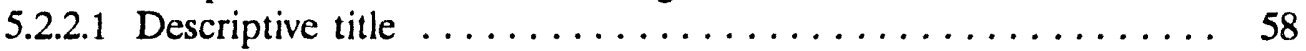

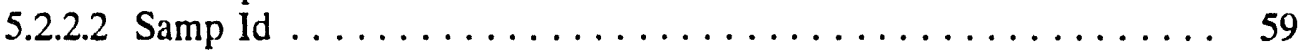

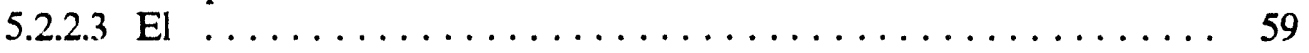

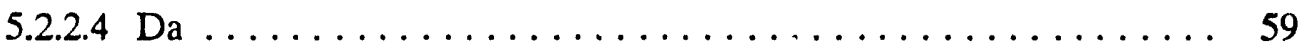

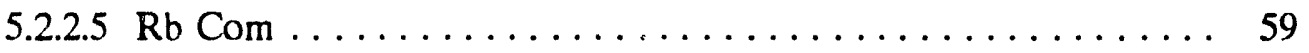

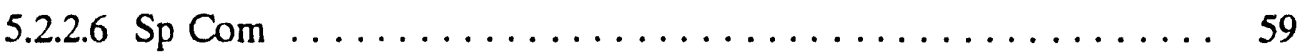

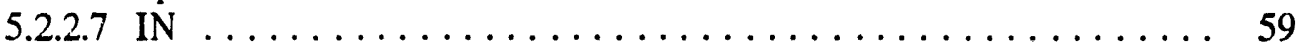

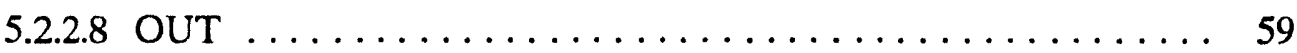

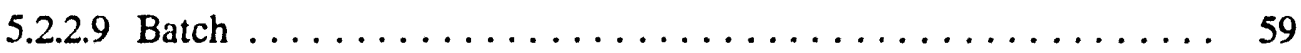

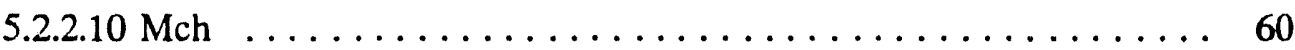

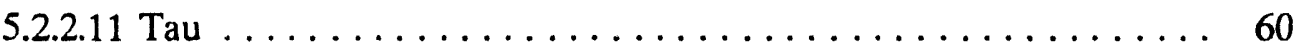

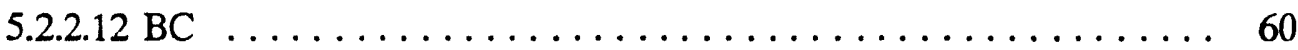

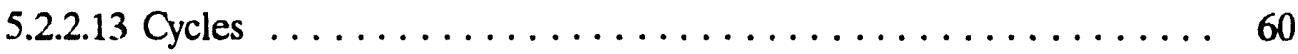

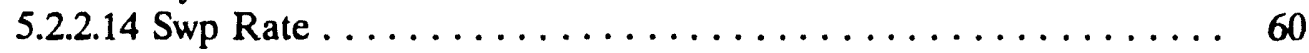

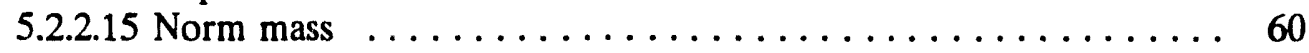

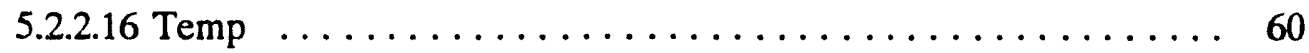

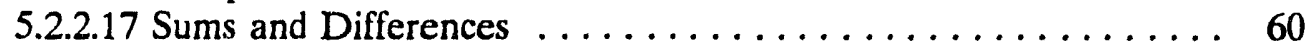

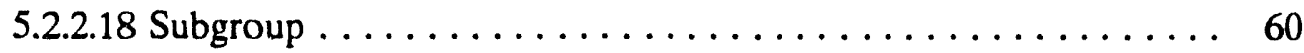

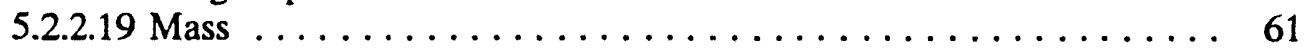

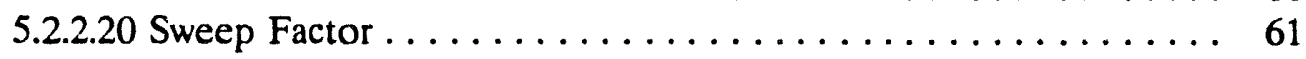

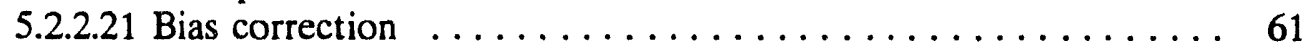

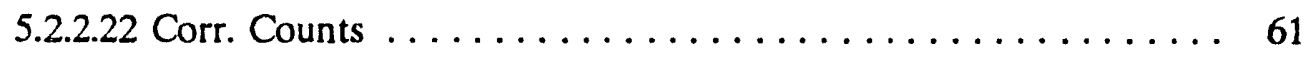

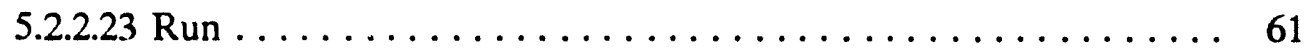

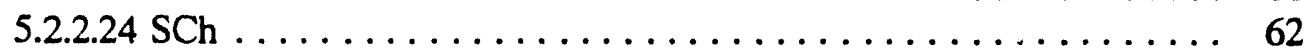

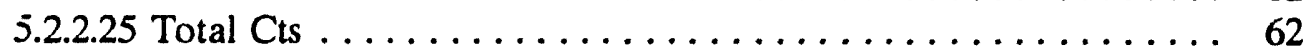

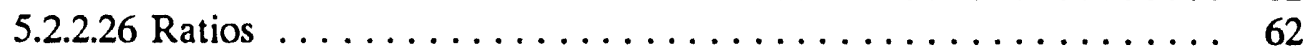

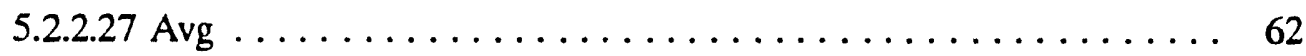

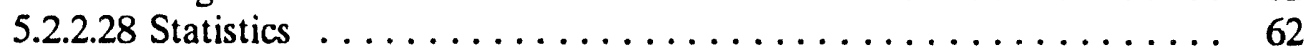

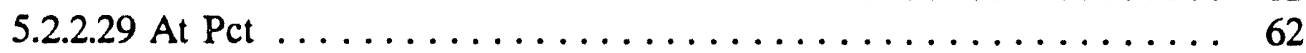

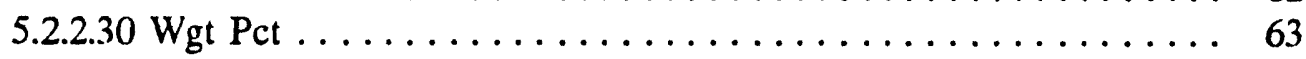

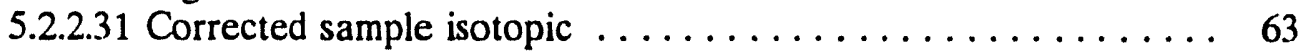

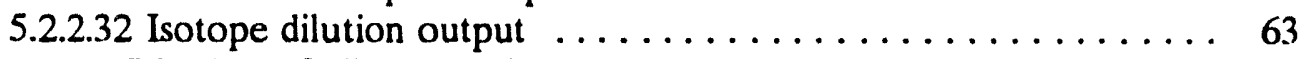

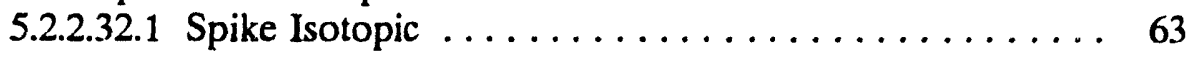

5.2.2.32.2 Other spike parameters $\ldots \ldots \ldots \ldots \ldots \ldots \ldots \ldots 63$

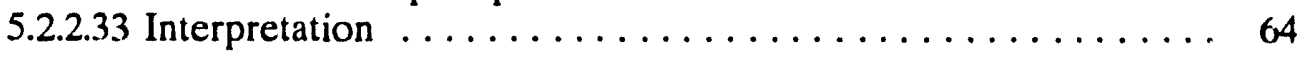

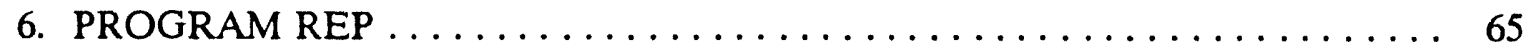

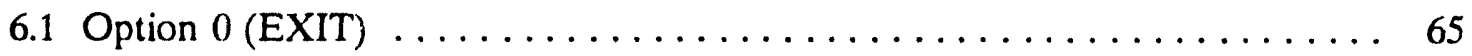

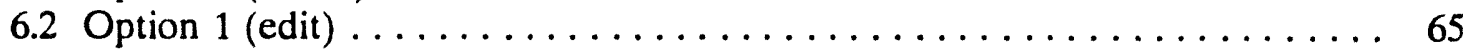

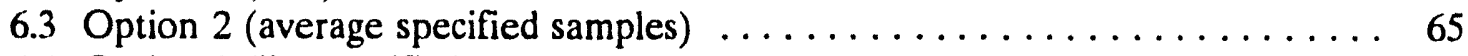

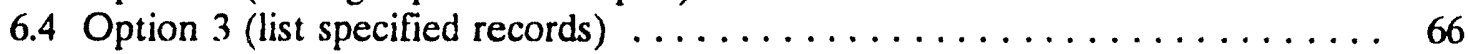

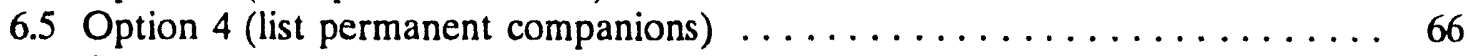

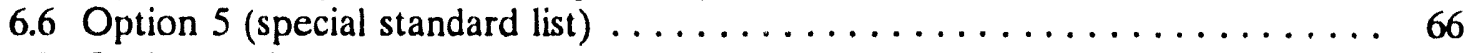

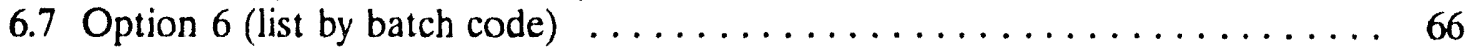

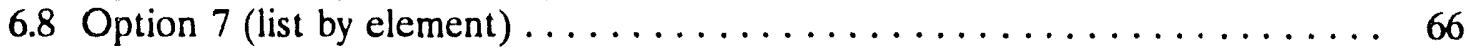


6.9 Option 8 (list sample id codes only) $\ldots \ldots \ldots \ldots \ldots \ldots \ldots \ldots \ldots \ldots$

6.10 Option 9 (list all between limits) $\ldots \ldots \ldots \ldots \ldots \ldots \ldots \ldots \ldots \ldots$

6.11 Option 10 (correct Pu to specified date) $\ldots \ldots \ldots \ldots \ldots \ldots \ldots \ldots \ldots$

7. PROGRAMS TRAN AND REC $\ldots \ldots \ldots \ldots \ldots \ldots \ldots \ldots \ldots \ldots \ldots$

8. MISCELLANEOUS PROGRAMS $\ldots \ldots \ldots \ldots \ldots \ldots \ldots \ldots \ldots \ldots \ldots \ldots \ldots$

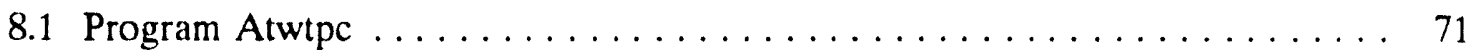

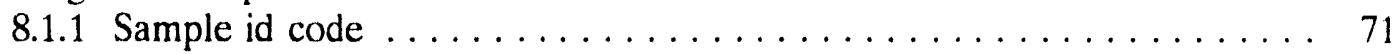

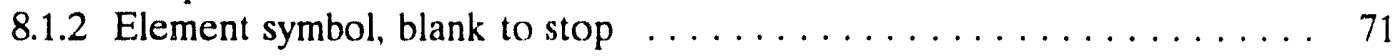

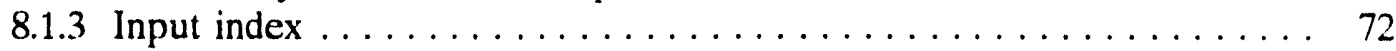

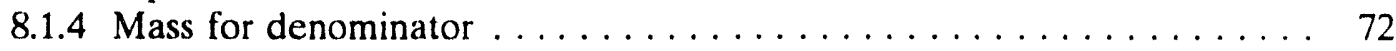

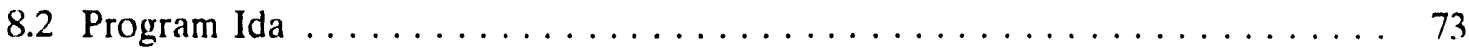

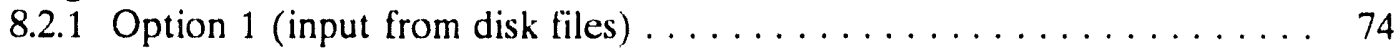

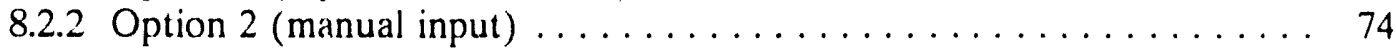

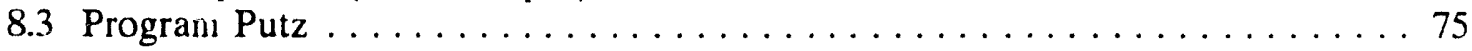

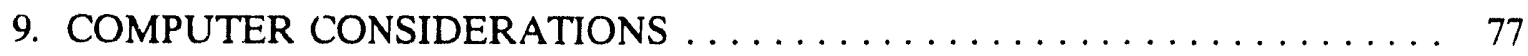

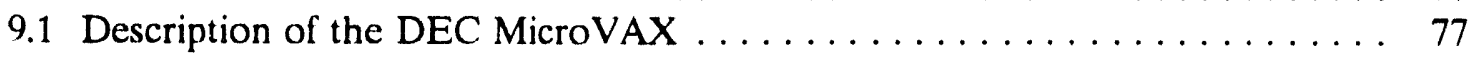

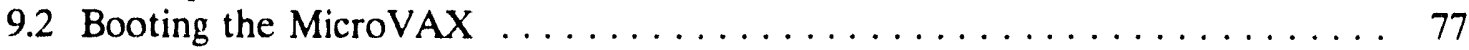

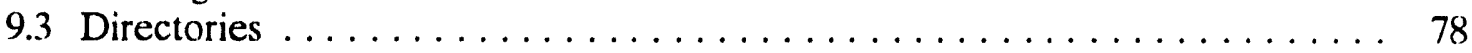

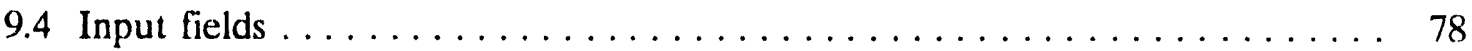

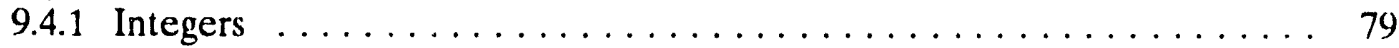

9.4 .2 Real numbers . . . . . . . . . . . . . . . . . . . . 79

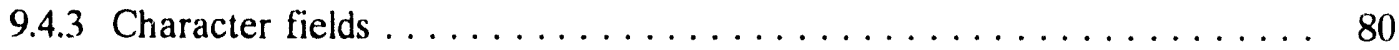

10. OPERATING A MASS SPECTROMETER $\ldots \ldots \ldots \ldots \ldots \ldots \ldots \ldots \ldots \ldots$

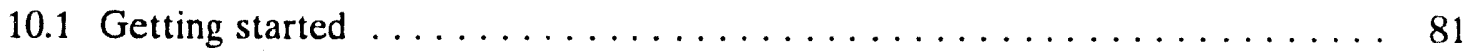

10.2 Using MSR $\ldots \ldots \ldots \ldots \ldots \ldots \ldots \ldots \ldots \ldots \ldots \ldots \ldots \ldots \ldots \ldots$

10.2.1 The Sweep Control Panel . . . . . . . . . . . . . . . . . 81

10.2.1.1 Subgroup number and the $8-16$ subgroup switch . . . . . . 82

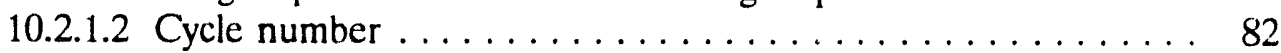

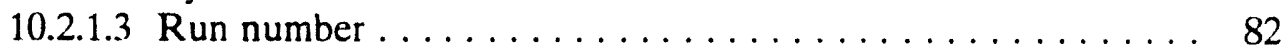

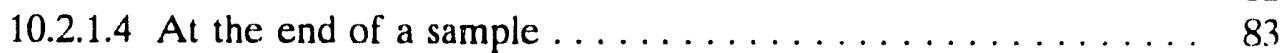

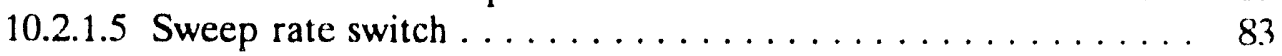

11. SPECIAL TOPICS $\ldots \ldots \ldots \ldots \ldots \ldots \ldots \ldots \ldots \ldots \ldots \ldots \ldots \ldots$

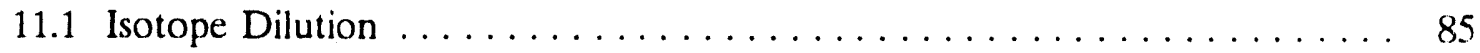

11.1.1 The isotope dilution equation $\ldots \ldots \ldots \ldots \ldots \ldots \ldots \ldots \ldots \ldots$

11.1.2 Example of the isotope dilution equation $\ldots \ldots \ldots \ldots \ldots \ldots$

11.1.3 Reverse isotope dilution . . . . . . . . . . . . . . . 87

11.1.4 Isotope Dilution with No Unspiked Companion . . . . . . . . . . 87

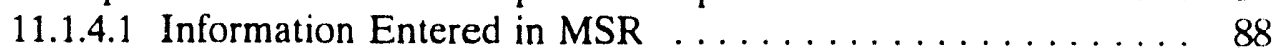

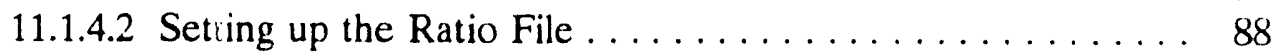

11.2 Internal Calibration $\ldots \ldots \ldots \ldots \ldots \ldots \ldots \ldots \ldots \ldots \ldots$

11.3 Instrument Calibration Calculations (NIST U-500) $\ldots \ldots \ldots \ldots \ldots \ldots . \ldots 9$

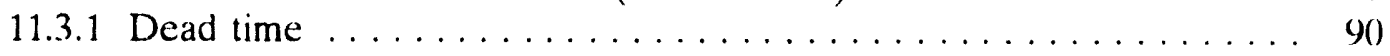

11.3.2 Bias correction . . . . . . . . . . . . . . . . . . 90 
11.3.3 How the program works . . . . . . . . . . . . . . . 91

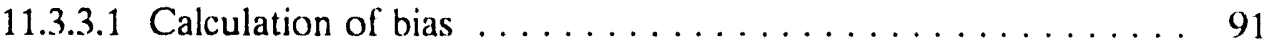

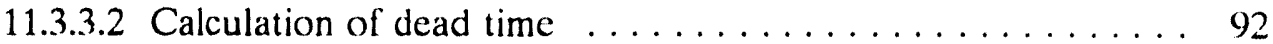

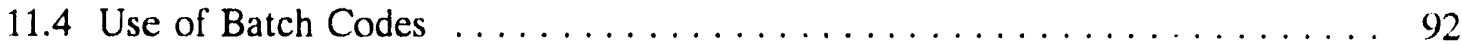

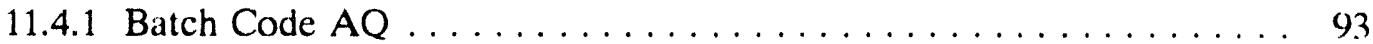

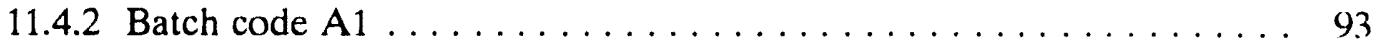

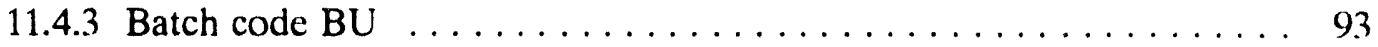

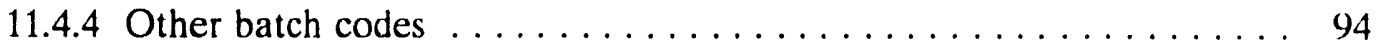

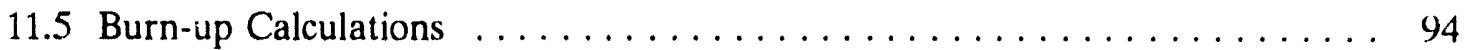

11.6 Mixed Uranium-Plutonium Analysis from Resin Beads . . . . . . . . . . 95

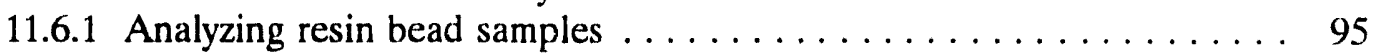

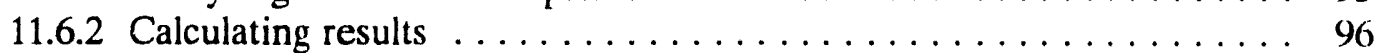

11.7 A Word on Statistics . . . . . . . . . . . . . . . . . . . . . . . 97

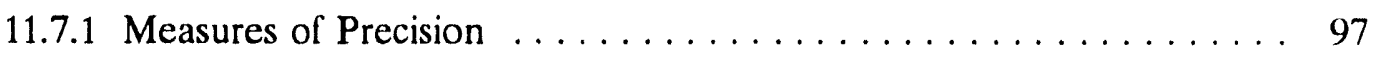

12. QUALITY ASSURANCE AND CONTROL . . . . . . . . . . . . . . 99

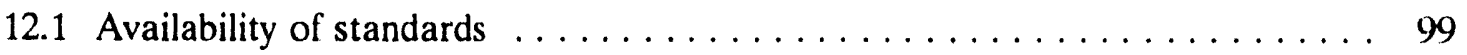

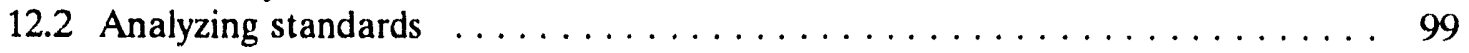

12.2.1 Instrumental calibration . . . . . . . . . . . . . . . . 100

12.2.2 Routine standards . . . . . . . . . . . . . . . . . . 100

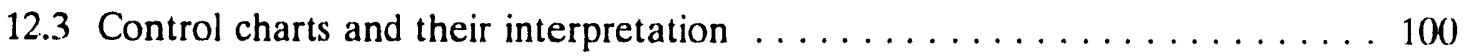

12.3.1 What to plot . . . . . . . . . . . . . . . . . . . 100

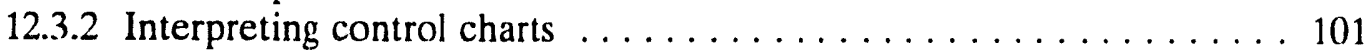

13. APPENDIX: EXAMPLES OF INPUT $\ldots \ldots \ldots \ldots \ldots \ldots \ldots \ldots \ldots \ldots \ldots$

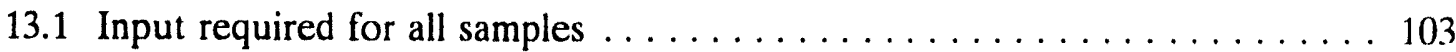

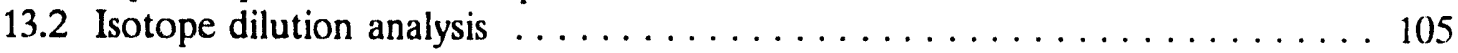

13.3 Isotope dilution analysis with no companion $\ldots \ldots \ldots \ldots \ldots \ldots \ldots \ldots$

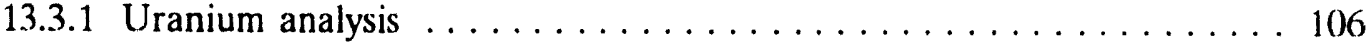

13.3.2 Thorium analysis . . . . . . . . . . . . . . . . . . . . . . 107

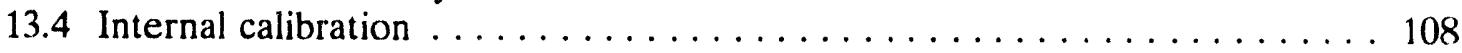

13.5 Mixed uranium-plutonium pairs $\ldots \ldots \ldots \ldots \ldots \ldots \ldots \ldots \ldots \ldots$

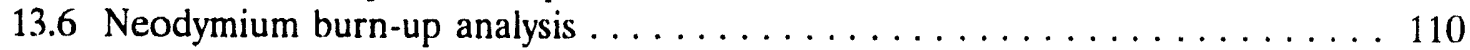

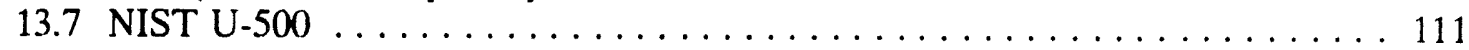

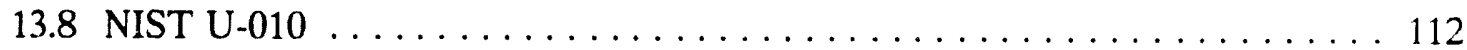




\section{LIST OF TABLES}

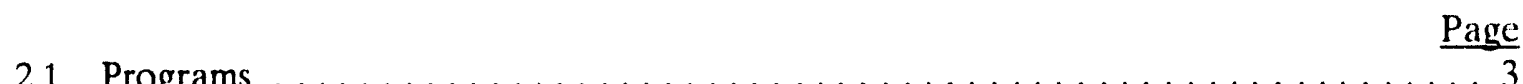

22 File Description $\ldots \ldots \ldots \ldots$

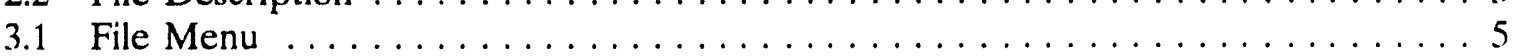

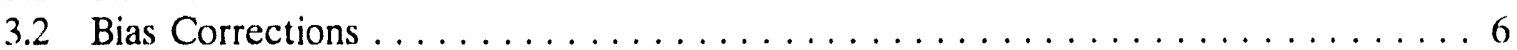

3.3 Definition of Instrument Numbers $\ldots \ldots \ldots \ldots \ldots \ldots \ldots \ldots \ldots \ldots \ldots$

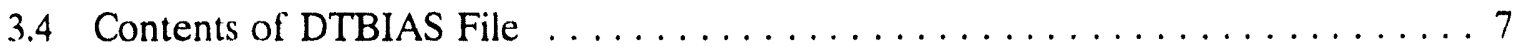

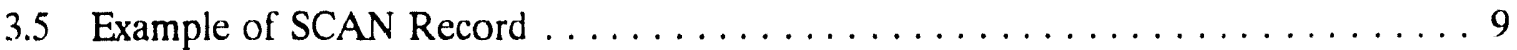

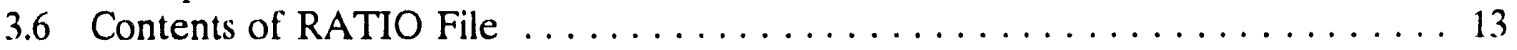

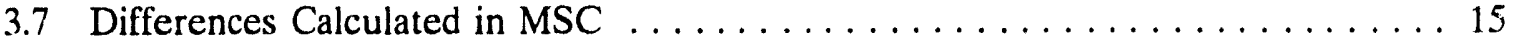

3.8 Specifying Sums and Differences for Ratio Calculations $\ldots \ldots \ldots \ldots \ldots \ldots \ldots$

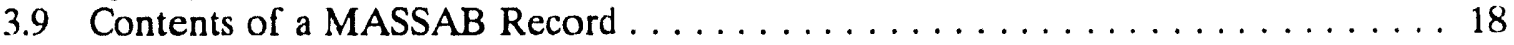

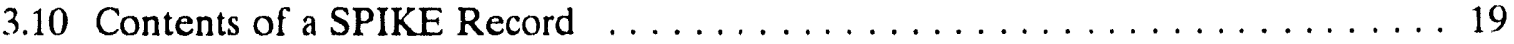

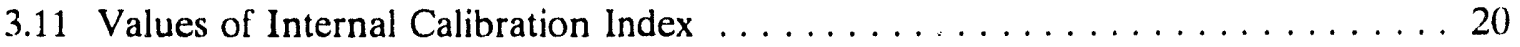

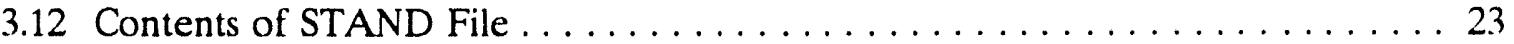

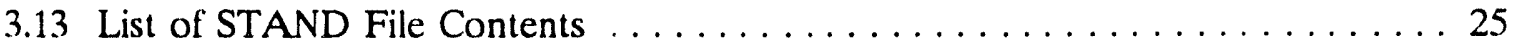

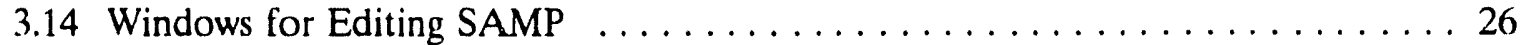

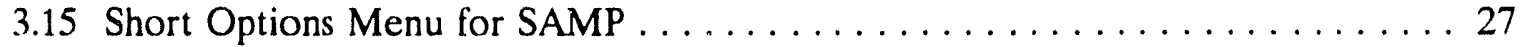

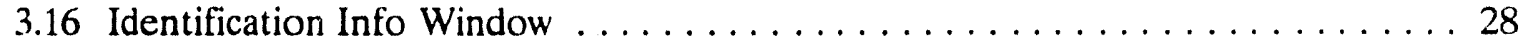

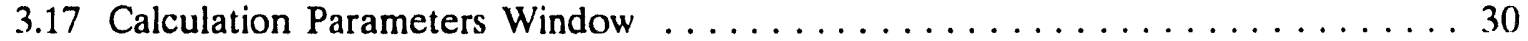

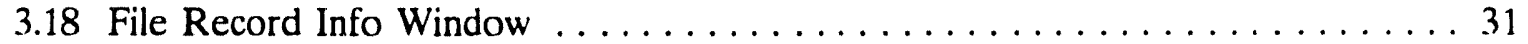

3.19 Mass Spectrometer Instrument Numbers $($ See 3.3$) \ldots \ldots \ldots \ldots \ldots \ldots \ldots, \ldots \ldots$

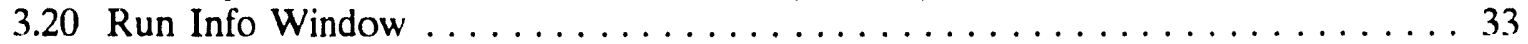

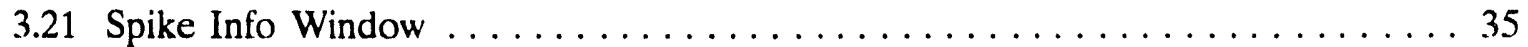

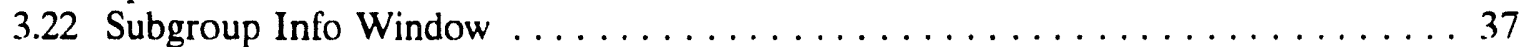

3.23 SUMCTS Window $\ldots \ldots \ldots \ldots \ldots \ldots \ldots \ldots \ldots \ldots \ldots \ldots, \ldots \ldots \ldots$

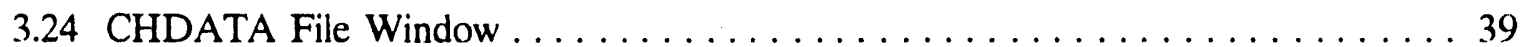

3.25 OUT File Contents . . . . . . . . . . . . . . . . . . . . . . . . . . . . . 40

4.1 Set-Up Window . . . . . . . . . . . . . . . . . . . . . . . . . . . 43

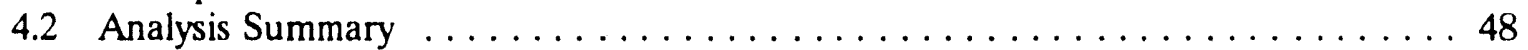

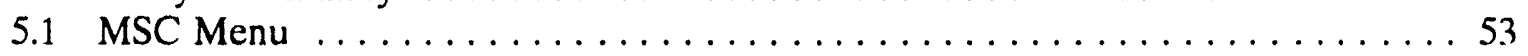

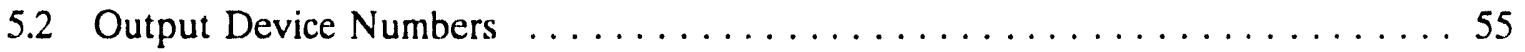

6.1 Rep Menu .................................... 65

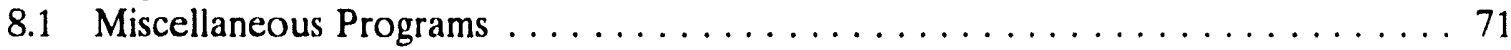

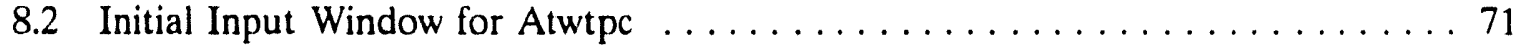

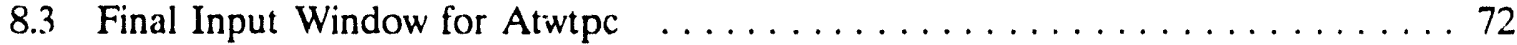

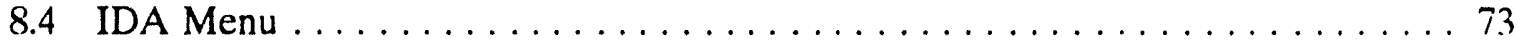

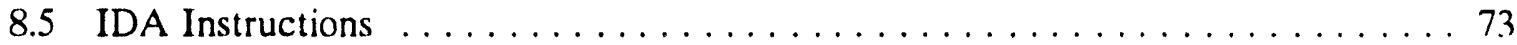

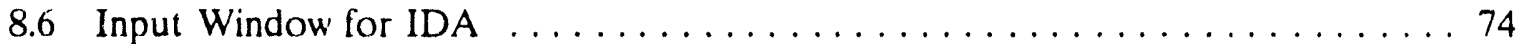

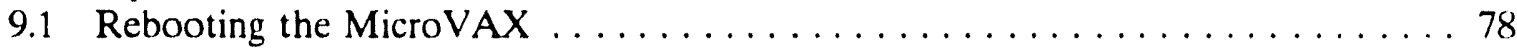

11.1 Sums for Uranium and Thorium $\ldots \ldots \ldots \ldots \ldots \ldots \ldots \ldots \ldots \ldots \ldots$

11.2 Batch Codes $\ldots \ldots \ldots \ldots \ldots \ldots \ldots \ldots \ldots \ldots \ldots \ldots \ldots \ldots \ldots \ldots . \ldots \ldots$

11.3 Setting up Burn-Up Calculations ......................... 95

11.4 Calculational Sequence for Mixed Resin Beads $\ldots \ldots \ldots \ldots \ldots \ldots \ldots . \ldots 96$ 


\section{PREFACE}

This is the third description of ORNL's isotope ratio computer programs to have received treatment as a Technical Memo. The first appeared in 1979, the second in 1982. The programs have been extensively rewritten since that time, primarily due to the acquisition of an entirely new data system. A DEC MicroVAX computer was obtained to replace our old PDP 11/23, and our old MINCs have been replaced with DEC 316 microcomputers, which are IBM PC clones.

This is one of a pair of new manuals that describe the new programs. This manual was written with the needs of the analyst in mind; the other was written for the computer programmer. It is the intent of this manual to lay out as lucidly as possible all the information an instrument operator needs for proper execution of the programs. Very little technical detail about the programs is given, although algebraic equations are presented when appropriate.

Note that there are two tables of contents. One is short and lists only chapter headings. The second is quite long and includes the heading for each individual section. It is hoped that the latter will serve as an index. The assumption is that the user will almost certainly know which chapter contains the information sought; scanning the section headings for one chapter should not be too onerous a task and should lead one to the right page number.

Variable, program, and file names are in italics. Program and file names are capitalized; variable names are not unless they begin a sentence. 


\begin{abstract}
This TM is one of a pair that describes ORNL-developed software for acquisition and processing of isotope ratio mass spectral data. This TM is directed at the laboratory analyst. No technical knowledge of the programs and programming is required. It describes how to create and edit files, how to acquire and process data, and how to set up files to obtain the desired results. The aim of this TM is to serve as a utilitarian instruction manual, a "how to" approach rather than a "why?"
\end{abstract}




\section{INTRODUCTION}

The Analytical Spectroscopy Section of the Analytical Chemistry Division is heavily involved in the isotopic analysis of many elements. Many analyses are performed to support nuclear-based projects, while others are generated by our support of ORNL's Stable Isotope program. Specialized mass spectrometers to perform these analyses were designed and built some years ago. These instruments consist of two or three stages to provide high abundance sensitivity and are equipped with pulse-counting detection systems for analysis of small samples ( $<1 \mathrm{ng}$ of uranium).

Back in the dark ages, data from these instruments were put out on punched paper tape in parallel with a strip printer. Channel information was laboriously entered by hand, and the tape, with its associated data sheet, shipped off to K-25 for proces ing. Many things could (and did) go wrong with this process: the tape tore, or the punch was out of alignment. or any one of seemingly countless things would cause us to lose our data. It was decided to purchase our own computer; we were trailblazers here, being the first lab in the Oak Ridge complex to have its own computer. Our first computer was an IBM 1130 with the grand total of $8 \mathrm{~K}$ of RAM (called core in those days). The first programs to process the data generated by the mass spectrometers were produced by Christie and McKown in 1971.

Replacement of the IBM 1130 with a DEC PDP $11 / 34$ necessitated rewriting the programs. The programs developed for the new computer were fundamentally very different from the originals and are described in the first edition of this manual. ${ }^{4}$ When in due course the PDP $11 / 34$ was replaced with an 11/23, a new version of the programs was written, and the second version of the manual appeared. ${ }^{5}$ This last system used DEC MINCs for datataking that were linked via a network to the central PDP 11/23.

Our present data system is far and away the most sophisticated and powerful we have ever had. A DEC MicroVAX with $13 \mathrm{Mb}$ of RAM (1625 times as much as the 1130 !) serves as the host for a local network linked via DEC's Ethernet. Each instrument has a DEC 316 personal computer that serves both as a node on Ethernet and as a controller of the instrument during data acquisition. From the operator's point of view, the software interface is not much different from that of the second edition (except everything is much faster!). The programs themselves, however, have been completely rewritten to conform, so far as practicable, to modern structured programming principles. This alone necessitated a new description for the programmer, and it seemed desirable to produce an operator-oriented manual at the same time. 


\section{OVERVIEW}

Four main programs and several lesser ones were developed to acquire and process mass spectral data. The most important programs are summarized in Table 2.1.

Table 2.1 Programs

\begin{tabular}{l} 
Program \\
\hline MSR \\
MSC \\
FILE \\
REP
\end{tabular}

Function

Acquiring data.

Processing data.

Editing files.

Generating reports.

In the mnemonics of the programmer, MSR stands for Mass Spec Run, MSC for Mass Spec Calculate, and Rep for Report.

The system of programs is based on a number disk files; each program accesses files as needed, allowing them to share the same data base. It is important for the operator to have at least a superficial knowledge of these files, how they work, and the information they contain. All these files are random-access, as opposed to sequential, in organization. This means that one may access an individual batch of data no matter what its location in the file; such batches are called records and are defined by the programmer. Sequential files can be read only from the beginning, so, if what you want is near the end, you will waste a lot of time reading the data ahead of it in the file. The files in question are listed in Table 2.2, along with short descriptions.

\section{Table 2.2 File Description}

File
SCAN
SAMP
CHDATA
SUMCTS
RATIO
SPIKE
STAND
MASSAB
DTBIAS
OUT

Contents

Scanning schemes for each instrument.

Basic information for each sample.

Raw channel data.

Condensed raw data.

Controls calculation of ratios.

Spike information.

Standard information.

Elemental information.

Dead time and bias information.

Results.

These files fall into two groups: ones whose contents remain relatively constant (Spike, Ratio, Stand, Massab, Dtbias, Scan) and those whose contents change with each sample (Samp, Chdata, Sumcts, Out). 
Access to the files is most often achieved through the record number associated with the data you want. This information can be gained in several ways; listings of current file contents can be obtained for any of the more static files, and information about each sample's record number appears on the output sheet for the more dynamic ones. The files are described in more detail in the next chapter.

\section{$2.1 \quad$ User Interface}

An attempt has been made to develop a user interface that is consistent between programs. Upon first entering a program, a window is opened with a menu listing the options available. The user chooses an option by entering the appropriate number in a special window devoted to the purpose. Some programs have several layers of such options before you reach the level where you enter new information; in each case a window opens with a menu of choices from which you make the appropriate selection. Option selection is always by a number associated via the menu with the desired choice. Entry of a zero usually causes the program to revert back one layer in the chain of menus and, ultimately, to exit the program and return control to the monitor. Zeros for options need no: be explicitly entered on the VAX; simply hitting RETURN is sufficient.

Entry of data into a program is almost always through a special window. At the end of the input of each batch of data, you are given a chance to correct any erroneous entries. Each variable that can be altered has associated with it an index number. It might look like this:

\section{Title}

A prompt at the bottom of the screen asks for the index number of any variable that needs to be corrected; entering a value greater than zero will direct the cursor to the appropriate place in the window and accept input of the new value for the variable. In the example above, entering a 3 for the index would direct the cursor to the title line and allow you to enter a new value. Entering zero (or RETURN) for the index value indicates that all input is correct and tells the program to move on to the next step in its execution. 


\section{PROGRAM FILE}

Program File provides access to the various data files to allow entry of new information or correction of old. There are two versions of it: a new version on the VAX and an older one on the DEC 316's. Only three files are necessary for operation of the mass spectrometers, and access to these three is all that's provided on the 316 version of File. These three files are Scan, Samp, and Chdata. Thus. in most ways, File on the PC's is scaleddown version of that on the VAX.

\subsection{File on the VAX}

File on the VAX provides access to all data files except Scan. Its option menu is shown in Table 3.1.

\section{Table 3.1 File Menu}

0 to exit

1 for DTPIAS--dead time and bias

2 for SCAN (does not reside on the VAX)

3 for RATIO

4 for MASSAB--mass and abundance file

5 for SPIKE

6 for STAND--standard file

7 for SAMP--sample header file

8 for SUMCTS or CHDATA--raw data files

9 for OUTput file

Entering your choice of option activates a second window that prompts you for your choice of function. These will be described as each file in turn is covered.

3.2 File on the DEC 316's

The original menu on the screen after invoking File on a PC looks like this:

Files to be viewed or changed:

Enter 0 to clock out

1 for SCAN

2 for SAMP

3 for CHDATA

Any choice but 0 (zero) will access the indicated file (described below). Entering 0 exits the program. 


\title{
3.3 Dtbias File
}

Dtbias is short for dead time and bias. Each instrument has associated with it a dead time and four bias corrections.

\subsubsection{Dead time}

Dead time is a function of the counting system and is a measure of the time the system is paralyzed after registering a count. Dead times are usually from 10 to $20 \mathrm{nsec}$, but may fall somewhat outside that range on occasion. Note that dead times are entered in units of microseconds. If the dead time in questions is $15 \mathrm{nsec}$, the correct entry would be 0.015 .

\subsubsection{Bias corrections}

Bias corrections arise from the discriminations inherent in the system. Every stage of generating a mass spectrum has associated with it a certain amount of bias that is massdependent. Bias is introduced in the evaporation process through isotopic fractionation, in the extraction of ions through the source, in transit through the flight tube, and in conversion to a pulse of electrons at the first dynode of the multiplier. It is not possible experimentally to measure these contributions individually, but it is tantamount to certain that the extraction process is the biggest contributor to bias. This is because the mass spectrum is scanned by sweeping the high voltage on the source. Each mass thus experiences a different extraction field, and different extraction fields capture ions with different efficiencies.

Bias correction is assumed to be linear with mass; this is an approximation that is valid over the relatively short mass range covered by the isotopes of one element, but is not valid over longer spans. For this reason four different bias corrections can be stored for each mass spectrometer; each of the four is applicable to a different mass region. Table 3.2 defines how these different biases are applied.

\section{Table 3.2 Bias Corrections}

\begin{tabular}{l} 
Bias No. \\
\hline 1 \\
2 \\
3 \\
4
\end{tabular}

\author{
Application \\ Below mass 100; extra bias for $\mathrm{Pu}$ \\ Mass $100-150$ \\ Mass $150-200$ \\ Over mass 200
}

The mass range used most often in our laboratories is above 200 , so the fourth value of the bias correction is applied. It has been experimentally determined that plutonium loaded as a solution requires more bias correction than when it is loaded on a resin bead. The extra bias to be applied is stored as bias (1); the need for this bias is signaled by use of batch code AQ. (See Section 3.9.2.8 for an explanation of batch code.) 
Typical biases range from about $0.25 \% /$ mass to about $0.50 \%$ /mass; they can fall outside this range on occasion. They are measured each time a multiplier is changed and monitored by routine analysis of certified NIST (nee NBS) standards (usually NIST U-010). Not all elements have certified isotopic standards available. An area of particular concern is the rare earth region; no standards are available for these elements. The best that can be done is to use the natural element and assume its composition is that listed by the International Union of Pure and Applied Chemistry.

\subsubsection{Instrument number}

Inspecting the Dtbias file is accomplished by selecting option 1 from the main File program menu (see Table 3.1). A window opens on the screen with the message:

Mass spec no., 0 to stop

Enter the number designating the instrument you are interested in as defined in Table 3.3 .

\section{Table 3.3 Definition of Instrument Numbers}

1. Three-stage mass spectrometer.

2. Two-stage mass spectrometer.

3. Single stage mass spectrometer (no longer active).

4. Transuranium mass spectrometer.

The instrument designated as number 3 is no longer on the network; it is now performing research in resonance ionization mass spectrometry in Building 4500S. Note that dead time is in units of microseconds and bias in units of percent per mass. It is essential to use the correct units for correct processing of the data.

\subsubsection{Contents of DTBIAS}

Upon entry of the instrument number, the appropriate record in the disk file is read and its contents displayed in a new window. Table 3.4 lists a typical example.

Table 3.4 Contents of DTBIAS File

1. Dead time in microseconds

0.0103

2. Bias $1, \mathrm{pct} / \mathrm{mass}$

$0.2600(0)$

3. Bias 2, pct/mass $\quad 0.260000$

4. Bias 3, pct/mass $\quad 0.500000$

5. Bias 4 , pct/mass $\quad 0.377000$ 


\subsubsection{Editing DTBIAS}

Below this window, in a window of its own, is the prompt:

Index no. to correct, 0 to proceed

The index number is listed at the far left side of the contents window. To change the value of one of the variables, enter the corresponding index. The cursor will move to the appropriate place in the contents window and signal for an entry. Enter the new value; the cursor will return to the index window to accept a new index value. This cycle will be repeated until an index value of 0 is chosen, upon which both windows will close and control return to the original File menu (Table 3.1).

As an example, suppose you want to change the dead time for the instrument in question. Enter 1 for the index; the cursor will move to the top line in the contents window. Enter the new value for the dead time (in microseconds); the cursor returns to the index window. This cycle repeats until an index of 0 is entered.

\section{$3.4 \quad$ SCAN FILE}

The Scan file controls data acquisition. It resides only on the PC's; there is no need for a VAX version since the VAX is not interfaced to a mass spectrometer. There is a different Scan file on each instrument, and their contents will vary from one instrument to the next. The fundamental structures, of course, are identical, but the details of the records will be different. This is because each instrument has a different sample load; a Scan record for californium, for example, would be superfluous on any but the transuranium mass spectrometer.

\subsubsection{SCAN menu}

Remember it is pointless to try to access Scan on the VAX; the ability to do so is retained only for program development and debugging. Upon entering 1 as your option from File on a PC, the Scan menu appears. It looks like this:

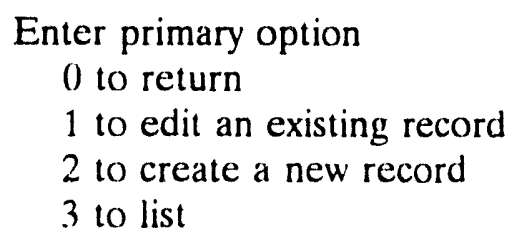

\subsubsection{Editing SCAN}

To modify or inspect a record already in the file, enter 1 . You will be asked to enter a record number, so you must know which record you want to edit. The contents of that record are listed; an example of the resulting display is shown in Table 3.5. 
Table 3.5 Example of SCAN Record

1. Element symbol

$\mathrm{U}$

2. Highest subgroup no.

8

3. No. of cycles

150

Ratios to be listed during analysis

\begin{tabular}{cclcc}
$4.234 / 238$ & \multicolumn{3}{c}{$5.233 / 238$} & $6.234 / 235$ \\
SG & Inx & Mass & Inx & SF \\
1 & 7. & 315 & 8. & 1 \\
2 & 9. & 233 & 10. & 1 \\
3 & 11. & 234 & 12. & 1 \\
4 & 13. & 235 & 14. & 1 \\
5 & 15. & 236 & 16. & 1 \\
6 & 17. & 365 & 18. & 0 \\
7 & 19. & 238 & 20. & 1 \\
8 & 21. & 239 & 22. & 1
\end{tabular}

The meaning of each variable is given below.

\subsubsection{Element symbol}

The element symbol should be self-explanatory. Ahout all that needs to be pointed out is that it is not necessary that it actually be the symbol of an element. It can be any twocharacter code. I suggest, however, that you be sure of what you are doing if you don't use an element symbol. An example of the use of a symbol that doesn't represent an element is the use of $A C$ to designate a mixture of americium and curium for the TRU mass spectrometer; note that $A c$ is the symbol of actinium, an element we have never analyzed. Americium and curium are extremely difficult to separate chemically, so they are analyzed from a single filament. Use discretion if you are choosing a new symbol. Do not, for example, use $\mathrm{Pt}$ as a second symbol for plutonium; you will get platinum.

\subsubsection{Highest subgroup number}

The highest subgroup number is the number of the highest subgroup to be swept for this analysis. It is most often 8 or 16 , but can be any value less than or equal to 16 . It is 4 , for example, for thorium analyses. Be aware of the distinction between the highest subgroup used and the number of subgroups swept. In the example (Table 3.5), the number of the highest subgroup is 8 , but only 7 subgroups are swept (subgroup 6 has a sweep factor of 0 ). Setting either the mass or the sweep factor associated with a subgroup to 0 will cause that position not to be swept during the analysis.

\subsubsection{Number of cycles}

The number of cycles defines the number of complete mass spectra that comprise a single run. It is usually chosen so that the time required for one run is about 90 seconds, but 
this isn't necessary: You can choose any number you like up to 999 . Experience has shown that 15 to 20 minutes is about the right length of time for a complete analysis to take; since 10 runs usually make up an analysis, the time for one run should be 1.5 to 2 minutes. There are times when you will not want to follow these guidelines, and the program is flexible enough to accommodate most reasonable desires.

Upon creating a new record, the program calculates the number of sweeps required for a 1.5 minute run. This is inserted during, entry of original data, but can be changed through use of the edit index.

\subsubsection{Display ratios}

The values of three ratios can be displayed for each run during the analysis. These are diagnostic to some degree of how well the analysis is progressing, so the ratios should be chosen with that in mind. Note that, when values for the masses of these are changed, they are entered in 213 fields. This means that to call for the $234 / 235$ ratio, for example, you should enter 234235 (no /, no spaces). The / is added to the display for ease of reading.

If the identities of the ratios displayed are of no consequence, default ratios can be called for by entering zeroes for numerators and denominators. This results in the most intense peak being assigned as the denominator for all three ratios; the three next most intense peak will be the numerators.

Displaying these ratios allows the analyst to monitor the progress of an analysis. The count rate is displayed for each run, so the stability of the signal can readily be assessed. Values of the ratios displayed for each run allow isotopic fractionation to be evaluated from their reproducibility as the analysis proceeds. The decay of a contaminant can also be monitored in this way.

\subsubsection{Masses and sweep factors}

The bottom part of the display is devoted to the masses and sweep factors for each subgroup. Each mass and sweep factor has its own index, ranging from 7 to a maximum value that is a function of the highest subgroup number $(22$ in the example above for 8 subgroups). The sweep factors define how many times each mass position is swept for a single traversal of the mass spectrum. Numbers larger than 1 improve the counting statistics for those mass positions. It is often desired, for example, to sweep minor isotopes more times than the major. It is common to analyze uranium using 4 or 8 for sweep factors for 234 and 236; 235 might have a sweep factor of 2 , while 238 , the major peak, remains 1 .

When choosing sweep factors, keep in mind the goal of the measurement to be made. Increase the sweep factors for isotopes of low abundance if they are important. In analyzing a NIST U-500 calibration standard, for example, it is crucial to obtain a good $234 / 235$ ratio so that a reliable dead time is obtained. A sweep factor of 8 is usually used for 234 in this case.

Remember, too, that the precision to which a measurement can be made is proportional to square root of the number of counts collected; you cannot do better than this. 
but you can do much, much worse. If the ratio is much less than 1.0, it is the minor peak that dominates the precision. With this in mind, we usually use sweep factors that are powers of two. Doubling the sweep factor improves the best precision obtainable by a factor of 1.4 . Striving to improve precision less than this seems not to be worthwhile, so we usually use sweep factors of $1,2,4$, or 8 . On rare occasions we use 16 and have been known to use 32 under exceptional circumstances. There is no reason, though, why you cannot use whatever numbers suit your fancy as long as they reflect the goal of the analysis.

\subsubsection{Changing a value}

To modify any of the variables listed in Table 3.5, enter the corresponding index number. To change, for example, the value of the third ratio to be listed during analysis, enter 6. The cursor will move to the right place on the screen and await entry of a new value. In this case, enter it in 213 fields (see above); the cursor will return to the appropriate place in the contents window and ask for a new value. And so on until you enter zero.

\subsubsection{Creating a new record}

To create a new record in the Scan file, enter 2 for the primary option. The program will lead you step by step through each parameter listed in Table 7, except for the number of cycles. It will calculate a suggested value for this parameter and list it on the screen. If you prefer a different value, change it following the instructions above for editing a record. Entry of new data terminates when a 0 is entered for the mass of a subgroup. If you don't want to sweep a mass that is in a subgroup lower than the highest one, enter 0 for the sweep factor; a non-zero mass number is needed, and you may choose whatever value you like--it can be a complete phony like 789. In Table 3.5, 365 was used to stand for mass position 236.5; it was necessary at one time to monitor this position to correct for tailing of mass 238 into mass 236 during uranium analysis, but improvements in the vacuum system rendered this unnecessary. As set up in Table 3.5, this Scan record will cause subgroup 6 to be omitted during data-taking.

\subsubsection{Listing the contents of SCAN}

Entering 3 for the primary option from the Scan menu allows you to list the contents of the file on either the screen or the printer. Note that Ethernet must be running to allow access to the printer (i.e., Startup should be executed before invoking File). Choose either $\mathrm{S}$ (for screen) or $\mathrm{P}$ (for printer) at the prompt. You will then be asked to enter an element symbol to list; it you want to list all entries, a blank (entered by simply hitting the return key) will accomplish it. It is strongly recommended that a current list of the Scan file be kept in a three-ring binder at each instrument.

\subsection{Ratio File}

The Ratio file resides only on the VAX. The purpose of this file is to tell program $M S C$ which isotopic ratios are to be calculated, which masses are to have atom percents calculated, which mass is the background and is to be subtracted from all other mass positions, and several other parameters necessary for successful calculations. It is accessed by selecting 
option 3 from the main File menu. Upon entering this routine, a window is opened with the Ratio menu listed on it. It looks like this:

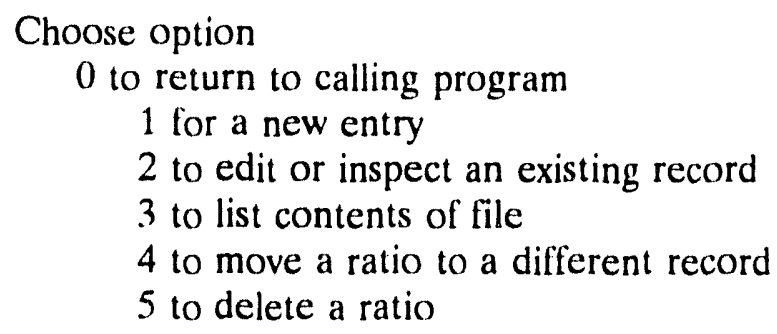

These options are described below.

\subsubsection{New ratio}

There is room for 300 ratios in the file, and several people will be entering new ones as their experiments demand. For this reason, when you choose to enter a new ratio, a list appears of the first 64 empty record numbers. Warning: Don't be impatient! It takes the computer awhile to locate the empty records. You need not choose one of these records, but the list saves you the trouble of identifying which ones are empty. It is convenient to locate all the ratios for the same element in the same region of the file, although this is by no means necessary. After you enter your record number, an entry window is opened, and you are stepped through the variables in sequence, entering values as appropriate. Table 3.6 lists the contents of one the records. Values listed in the taule as "No entry" appear as blanks on the screen; their appearance in the table is an attempt to make the entries in it as clear as possible.

\subsubsection{Contents of a RATIO record}

\subsubsection{Element symbol and record number}

The top line of the output lists the element symbol and which record in the Ratio file you are addressing. As in the Scan file, the element symbol need not be one of a real element, although that is almost always the case. The reason for choosing a different symbol usually arises from analyzing a sample whose isotopic composition differs from that of the naturally occurring element. The Massab file (see Section 3.6) holds the isotopic masses and abundances of all naturally occurring isotopes and the masses of some commonly encountered man-made ones; most of these latter are for the transuranium elements. If you do decide to create an element symbol, be careful not to choose one already in use. For example, to use $P$ when you want lead will infallibly give you phosphorus.

\subsubsection{Base mass for bias correction}

The next entry lists what we call the base mass for bias correction. Application of bias correction is arbitrary in that any isotope can be defined as having a bias of 1.00000 . All other isotopic biases are then calculated in reference to it, subtracting or adding the bias correction per mass depending on the mass of the istope in question. We have traditionally 
subtracted bias, so $238-\mathrm{U}$ is usually assigned a bias of 1.0 ; for plutonium, mass 242 is assigned a bias of 1.0. There are exceptions to this that are covered in other sections of this manual.

\section{Table 3.6 Contents of Ratio File}

1. Element symbol

2. Base mass for bias correction

3. Normalizing mass for atom pcts

4. Mass to be subtracted from all SGs

5. Sum No. 1

6. Sum No. 2

7. Sum No. 3

8. Differ-nce No. 1

9. Difference No. 2

10. Difference No. 3

Ratios 1-11

11. $233 / 238$

12. $234 / 238$

13. $235 / 238$

14. $236 / 238$

15. $234 / 18$

16. $235 / 18$

17. $236 / 18$

18. $238 / 18$

19. $238 / 233$

20.

21.
U

Record no.
14

238

238

315

$23.3+234+235+236+238$

$234+235+236+238$

No entry

No entry

No entry

No entry

Ratios 12-22

22.

23.

24.

25.

26.

27.

28.

29.

30.

31.

32.

\subsubsection{Normalizing mass for bias correction}

Most of our analyses require calculation of atom percents for reporting to our clients-only mass spectrometrists think in ratios! The program must of course know which masses to include in the calculation of these percents, and it does so by using the masses included in sum(1). If no atom percents are desired, enter 0 for the normalizing mass. Atom fractions are explicitly carried through the calculations, using the mass of sum(1) for the numerators and their sum as the denominator. Use any appropriate mass for this parameter.

\subsubsection{Mass to be subtracted from all other SG's}

This is a background position whose sole function is to measure the electronic noise in the system. As the message says, the counts in this subgroup are subtracted from those of all other subgroups to correct them for background. The subgroup corresponding to this mass position must be positioned so that no part of a peak is covered by it. Be careful not to get it so close to a peak that counts from the peak tail into it. Be careful not to choose a mass that has a contaminant peak; for example, mass 232 should not be used during a uranium 
analysis because thorium will fall at this position. The best thing to do is to set the subgroup at a half-mass position well removed from the location of strong ion signals.

The mass assigned to the background subgroup should be chosen with a little care. $M S C$, the calculational program, will not apply a bias correction to any mass that differs from the base mass (Section 3.5.2.1) by more than 10; this is what is wanted in this case--it makes no sense to apply a bias correction to background noise. With this in mind, choose an integer of up to three digits that differs from the base mass by more than 10. For example, 315 (for mass 231.5) is commonly used for uranium. There is no reason that a meaningless mass number can't be used--666, for example--as long as that number is the one always used to refer to the background position.

\subsubsection{Sums}

MSC allows up to three sums to be used, although it is not necessary to have even one. There are some constraints to using these sums that you need to be aware of. The most important is that MSC assumes that the masses in $\operatorname{sum}(1)$ are to be used to calculate atom percents. It carries through the calculations ratios calculated by dividing the counts for each mass listed in the sum by the sum of those counts--the atom fractions, in other words. There is no way to suppress this. If you want to use a sum without having atom percents calculated, enter 0 for the normalizing mass, or use $\operatorname{sum}(2)$ or $\operatorname{sum}(3)$. It is not necessary to use $\operatorname{sum}(1)$ to use $\operatorname{sum}(2)$, and Sum 3 can be used whether or not there are entries for sum(1) and $\operatorname{sum}(2)$.

Another special case needs to be mentioned; this is also covered in Section 11.1.4. For isotope dilution samples where no unspiked analysis is performed, a spike companion of $\mathrm{NO}$ is entered. Isotope dilution calculations are performed on the analysis of the mixture of spike and sample on the assumption that there is at least one mass in the spike that is not present in the sample. This is a good assumption for uranium, where 233 is rarely present in samples. Another common application of this feature is in thorium analyses, where the 230 spike isotope does not occur in the sample. The program identifies this mass by finding which mass appears in sum(1) that does not appear in sum(2). In the example in Table 3.6, this would be 233 . Thus the rule is:

Use Sum 1 for sample plus spike. $(233+234+235+236+238)$

Use Sum 2 for the sample alone. $(234+235+236+238)$

Failing to follow these instructions will cause unpredictable results. This is one of the things you should check if concentrations are negative, as this is one possible manifestation of the problem.

\subsubsection{Differences}

Three differences can be accommodated by program MSC. As with the sums, it is not necessary to calculate a difference--in fact, it is rare to calculate even one. The reason is that most differences that need to be calculated are taken care of in other ways. Table 3.7 lists differences that are calculated without calling for one in the Ratio file. 


\section{Table 3.7 Differences Calculated in MSC}

1. Subtraction of background from all subgroups.

2. Correction of the mass 238 position when $\mathrm{U}$ and $\mathrm{Pu}$ are run from the same resin bead.

3. Correction of $\mathrm{Nd}$ for $\mathrm{Ce}$ contribution for a reactor burn-up analysis.

4. Blank correction for isotope dilution calculations.

5. Subtraction of the contributions of up to three contaminant elements.

All the cases in Table 3.7 are discussed in more ditail in the chapter covering MSC (Chapter 5).

The utility of differences calculated via entry of values for them in the Ratio file is further limited because they are calculated on one-to-one basis: Counts in the subgroups to be subtracted are removed directly from that of the first mass indicated with no multiplicative factor employed. This situation occurs only rarely. Except for the background, most corrections are for interferences of some kind; these interferences are almost always elemental in nature, and correcting for them requires multiplying by some factor to take into account the isotopic composition.

\subsubsection{Ratios}

These are the ratios that $M S C$ is to calculate. They are usually specified by mass; MSC automatically locates the corresponding subgroup positions. An exception is if you wish to calculate a ratio that has a sum or difference in it. There is no obvious way to identify the sum or difference involved; the one chosen seemed good at the time but has not received unanimous acclaim. As follows: A maximum of 16 subgroups can be swept; values 1-16 for specifying numerators and denominators for ratios are reserved for these positions. Sums and differences are specified by adding to 16 and 18 , respectively, as shown in Table 3.8 .

Table 3.8 Specifying Sums and Differences for Ratio Calculations

\begin{tabular}{lc} 
Value & Variable used \\
\cline { 2 - 2 } 17 & Sum 1 \\
18 & Sum 2 \\
19 & Sum 3 \\
20 & Difference 1 \\
21 & Difference 2 \\
22 & Difference 3
\end{tabular}


This feature is most frequently used to get an atom fraction of the unspiked element when no unspiked isotopic composition is available (See Section 3.5.2.5). Thus, for the uranium example used in that section, specifying $234 / 18,235 / 18,236 / 18$, and $238 / 18$ would give atom fractions. These are uncorrected for the contribution of the spike, but a corrected isctopic composition is printed near the bottom of the second output sheet.

Program MSC is dimensioned to handle a maximum of 22 ratios. This was the maximum that could be shoe-horned into the memory of our old PDP 11/34. Since we have never encountered a situation where 22 was too few, the maximum has remained fixed at that value. You need to keep in mind that calculation of atom percents automatically creates more ratios than indicated in the Ratio file. This is because it generates atom fraction ratios using the sum of counts of the masses in $\operatorname{sum}(1)$ in the denominator and counts of the indvidual masses so specified in the numerator. This creates atom fraction ratios that are subjected to the same statistical processing all the other ratios are. If you are analyzing uranium, 17 is the maximum number of ratios you should specify. This should cause no trouble; it is highly unlikely that so many ratios will be needed.

There are some occasions when it is more convenient to use default ratios than to enter explicitly those you want calculated. The Stable Isotopes program, for example, will often submit several samples of the same element, each with a different enriched isotope. This ordinarily would require a separate Ratio file entry-for each isotope, but using default ratios provides a concurrent way of avoiding this hassle.

To invoke default ratios, enter 0 for the numerator of the first ratio. This is the cue for the program to identify default ratios. Default ratios all have the most intense isotope included in sum(1) for their denominator. All other isotopes in sum(1) are used as numerators. The ratio of the background position to the must intense peak is also included. As an example, suppose you were analyzing a lead sample enriched in ${ }^{207} \mathrm{~Pb}$. The $\operatorname{sum}(1)$ should contain (you must have a sum(1) to use default ratios):

$$
204+206+207+208
$$

You will get the following ratios:

\section{$204 / 207 \quad 206 / 207 \quad 208 / 207 \quad 666 / 207$}

(assuming 666 is the background position).

There are other conditions that lead to extra ratios being added to those called for by the Ratio file. Isotope dilution calculations add one ratio to the list, and internal calibration calculations add several more. These are described in the appropriate sections of Chapter 5.

\subsubsection{Listing the Ratio file}

Entering a 3 as your selection for Ratio menu will generate a list of the contents of the file. It will go to the monitor screen or to the printer as you choose. 


\subsubsection{Moving a record}

It occasionally happens that it is desirable to move the contents of a Ratio record to a new one. This occurs, for example, when one wants to put all the entries for a single element in sequential records. This feature is more a convenience than a necessity. You are prompted to enter the record number of the information you want to move and a destination record number to which it will be moved. No further information is required. Note that the contents of the original record remain intact. See the next section for how to delete them.

\subsubsection{Deleting a record}

Deleting a record disables it from use. You are asked for the record number to be deleted--that's it!

\subsection{MASSAB FILE}

The name of this file, Massab, is intended to be a mnemonic for mass and abundance. This file holds the masses and abundances of all naturally occurring isotopes; it also holds masses for some isotopes (such as those of the transuranium elements) that do not occur in nature.

The file is organized by element; access to it is provided by searching element symbols in the file for a match to the one you enter. It is reached by entering 4 for the choice from the File menu. A window opens with a message like this:

0 to stop

1 to edit or inspect an element

2 to print entire file on printer

\subsubsection{Editing Massab}

Upon entry of a 1 in response to the above message, a new window opens, and you are asked to enter the element symbol. For single-character symbol (such as $U$ ), it doesn't matter if you enter the character right- or left-adjusted. The case (upper or lower) doesn't matter; the input routine converts all entries to capitals and right-adjusts them.

Table 3.9 contains an example of the contents of a Massab record. 
Table 3.9 Contents of a MASSAB Record

Record no.

1. Element symbol

2. No. of isotopes

Mass

3. 233.0395

5. 234.0409

7. 235.0439

9. 236.0456

11. 238.0508
83

$\mathrm{U}$

5

Abundance

4. 0.000000

6. 0.000055

8. 0.007205

10. 0.000000

12. 0.992740

The meaning of the several variables should be self-evident. I remind you that the variable called element symbol need not be a recognized element symbol; it usually is, but there are occasions when it is desirable to make a second entry for an element when an isotopic composition different from the original is desired. To correct an entry in the display illustrated above, enter the corresponding index. To change the isotopic abundance of mass 235 , for example, enter 8 . The cursor will move to the appropriate place on the screen and accept a new value. This process will continue until 0 is entered for the index value.

\subsection{Spike File}

The Spike file contains information pertaining to spikes--surprise! The information is needed for isotope dilution calculations. The file is organized by record number; it is accessed from $M S C$ by your having entered the appropriate record number when you were setting up on a PC to take data using MSR. Access to the file for editing is achieved by entering 5 for the option from the File program menu. A window thereupon opens that looks like this:

Select an option:

0 to return to calling program

1 for a new entry

2 to edit or inspect an existing record

3 to list contents of file on printer

4 to delete a spike

5 to update Pu-947

You enter your selection in the small window at the bottom of the screen. The various options are described below.

\subsubsection{New spike}

There is room for 100 spikes in the file. When a new spike is to be entered, the program searches the file for empty records and lists them on the screen. Be patient; this 
takes a little while. Unless you're sure you want to replace an existing spike, you should choose one of the record numbers listed. An example of a spike record is listed in Table 3.10. The table simulates the appearance of the editing screen.

\subsubsection{Contents of a SPIKE record}

Table 3.10 Contents of a Spike Record

1. Element symbol

2. Label for spike

3. Atomic weight

4. Internal calibration index

5. Isotope dilution ratio

6. Internal calibration ratio Mass

7. 233

8. 234

9. 235

10. 236

11. 238
$\mathrm{U}$

Record no. 5
NEW LOW LEVEL 233 SPK, 11-9-83
233.0411
0
$2.38 / 233$
0
Abundance
0.9996860
0.0000030
0.0000050
0.0000001
0.0003060

The meanings of the various entries are given below.

\subsubsection{Element symbol}

This again is self-explanatory. No checking is done in the program to be sure that the element symbol for the spike agrees with that for the sample. If a mismatch occurs, you will probably get gibberish for output. There is no reason I can think of to use the symbol that doesn't represent an element.

\subsubsection{Record no.}

This is the record number in the file that contains the information displayed. It cannot be changed from this window. If you entered the wrong record number, enter zero for the editing index. This will allow you to enter a new record number.

\subsubsection{Label for spike}

This variable provides a descriptive label of up to 40 characters for each spike. It is desirable to have the date the spike was entered included here. 


\subsubsection{Atomic weight}

This is the atomic weight of the spike. You do not need to calculate and enter it specifically; the program does it for you when information for the spike is first entered. You can edit it, but it seems unlikely it will ever be necessary to do so.

\subsubsection{Internal calibration index}

This index is used in internal calibration calculations. Its values are defined in Table 3.11 .

Table 3.11 Values of Internal Calibration Index

$\begin{array}{ll}\frac{\text { Value }}{0} & \text { Meaning } \\ 1 & \text { Internal calibration isotopes are in the spike. } \\ \text { Internal calibration isotopes are in the sample. }\end{array}$

The value will usually be 0 . A value of 1 is used mostly when the concentration of a double (internal calibration) spike is being calibrated; this allows us to take advantage of the improved accuracy and precision achieved by this technique. This situation is in some ways analogous to reverse isotope dilution (Section 11.1.3).

\subsubsection{Isotope dilution ratio}

This ratio defines the default ratio used in isotope dilution calculations. It is superseded if the analyst enters a different value when setting up for the analysis on a PC in $M S R$. Using the default values is a convenient way of avoiding having to enter it for every sample. An instance of where changing it would be desired would be if you were using a common uranium spike (like that described in Table 3.10), but the sample was so enriched that 235 was the major peak. You would want to enter 235/233 for the isotope dilution ratio when you were entering the sample information at the PC. If you forget to do this, it is always possible (and easy) to alter the value in the Samp file (Section 3.9) and reprocess the data.

In the example in Table 3.10, 238/23.3 would be used as the isotope dilution ratio unless another value was entered.

\subsubsection{Internal calibration ratio}

This is the ratio to be used in internal calibration calculations. It must be the ratio of the two enriched isotopes used to make up the internal calibration spike. For uranium, this ratio should be 233/236 and for plutonium 244/242. Note that the denominator in each case should be the base mass for bias correction in the Ratio file. Not to set the files up this way leads to small errors in the calculations. This is because, as an example, $0.994 / 0.997$ does 
not exactly equal $0.997 / 1.000$. To address this little problem, it is necessary to define the Spike and Ratio files as indicated.

\subsubsection{Masses and abundances}

This should be self-explanatory. The isotopic composition of the spike is contained here. Upon making a new entry, the program locates the element in the Massab file and lists the masses stored there as suggested isotopes. You may either accept or reject them. If you reject them, you must enter values for the isotopic masses of the isotopes; otherwise you need enter only the abundances.

\subsubsection{Editing and inspecting the SPIKE file}

Changing values in the Spike file is similar to doing so in other files. You enter the record number whose contents you want to change, and the present values of the variables are listed on the screen in a format like that of Table 3.10. You select the variable whose value needs changing by entering its index; the cursor moves to the appropriate place on the screen and waits for the new value to be entered. When this has been accomplished, a new index to be changed is requested. The program cycles in this way until a zero value for the index is entered; the editing routine is left at that point.

Note that this option can be used simply to inspect the contents of a record when there is no intention of changing them. The current contents are displayed on the screen; entering a zero for an index value after you have completed your inspection leaves the record unaltered. This is useful when trouble is encountered in the calculations and the validity of the spike information must be verified.

\subsubsection{Listing contents on the printer}

Selecting option 3 from the Spike menu results in obtaining a hard copy of the file on the printer. It is desirable to maintain a current listing of the file near the mass spectrometer so appropriate spikes can be identified without having to resort to the computer.

\subsubsection{Deleting a spike}

Selecting this option allows you to enter a record number whose contents are no longer needed. This will free the record for use by another spike.

\subsubsection{Updating Pu-947}

NIST (nee NBS) Pu-947 is a certified isotopic plutonium standard. 241-Pu has a halllife of about 14.5 years, which is short enough to change significantly on a human time scale. When the standard was originally certified on October 13, 1971, the abundance of 241-Pu was $4.540 \%$; on February 12, 1992, this value had dropped to $1.750 \%$. This latter value is not exact due to uncertainty in the half-life of $241-\mathrm{Pu}$.

This option allows the Spike file entry for this standard to be updated to whatever date you specify. Note that entry for the date is in 312 format; this means that today's date 
(as I write this) would be entered 080492--August 4, 1992. There must be no blanks, commas, dashes, etc., embedded in the string of numerals. The three numerals each must occupy two characters as emphasized here by the leading zeroes; blanks are also valid.

\subsection{STAND File}

The Stand file holds information for reference standards. It is used for two purposes: to allow easy access for printing an isotopic composition when a daily standard is run, and for instrumental calibration purposes. NIST (NBS) U-500 is the only standard so far used for the latter purpose. NIST U-010 and NIST Pu-947 are the most common daily standards.

Upon entering a 6 for the selection from the File program menu, a window opens with the Stand menu on it. It looks like this:

\section{Select option:}

0 to return to calling program

1 to insert a new entry

2 to edit or inspect a record

3 to list labels only

4 to list entire file

5 to edit last record used

These options are described below.

\subsubsection{New entry}

The record used for a new entry is defined by the value in the bookkeeping record for the last record used. This value is read, incremented by 1 , and assigned to the new entry. The new value of the last record used is written into the bookkeeping record. You should not have to worry about this little bookkeeping chore.

When you choose to enter a new standard, you are stepped through an input routine that asks for all necessary information required. An example of an editing window for Stand is shown in Table 3.12 . 
Table 3.12 Contents of STAND File

\begin{tabular}{lllll} 
Record no. 5 & Element & U & \multicolumn{3}{c}{ Label NBS U-020 } \\
Dead time ratio & $234 / 235$ & \multicolumn{3}{c}{ Bias ratio 235/238 } \\
Ratios & $234 / 238$ & $235 / 238$ & $236 / 238$ & \\
& 0.0000 & 0.0208 & 0.0002 & 238 \\
At Pcts & 234 & 235 & 236 & 97.9330
\end{tabular}

Edit Index

0 to stop

1 for label

2 for calibration ratios

3 for masses or at. pcts.

Ratios will be recalculated

The variables are described below.

\subsubsection{Contents of STAND}

It is not necessary to enter all the information displayed in Table 3.12; either the ratios or the atom percents are calculated from the other.

\subsubsection{Record number}

This is the record number whose contents are being displayed.

\subsubsection{Element}

The element symbol. No matching with the sample element symbol is performed.

\subsubsection{Label}

Provision is made for a descriptive label of up to 20 characters.

\subsubsection{Dead time ratio}

This variable is used only when an instrumental calibration standard is being run. It identifies the ratio to be used in calculation of the dead time. The entry for this variable in Table 3.12 is superfluous: It will not be used unless NIST U-020 is employed as an instrumental calibration. It would be ill-advised to do so because the abundance of $234-\mathrm{U}$ is so low that the necessary high precision measurement cannot be made. For NIST U-500, this ratio should be $234 / 235$. 


\subsubsection{Bias ratio}

Like the dead time ratio, this variable is used only when an instrumental calibration standard is being run. It identifies the ratio to be used in calculating the bias correction. Although a bias correction could be calculated from the standard listed in Table 3.12, it would be poor practice to do so. The bias correction is calculated before application of a dead time correction, which presupposes the ratio involved is reasonably close to $1.0 ; 0.02$ hardly meets this definition. For NIST U-500, our calibration standard, this ratio should be 235/238.

It is important to distinguish between calculation of bias correction and verification of it. Calculation is performed in a special part of the program dedicated to it alone; several conditions have to be met, such as the ratio being close to 1.0. NIST U-500 is used for this purpose. Verification is addressed by inspecting the standards run as routine quality control checks. The standard most often used for this is NIST U-010, but that is only because most of our samples have $1 \% 235-\mathrm{U}$ or so. Any of the NIST uranium standards can be used for this purpose. By keeping quality control charts of the results of these analyses, long-term trends can be identified and, if necessary, the bias correction revised to ar sommodate them.

\subsubsection{Ratios}

These are the isotopic ratios of the standard with the masses involved listed over them. When entering information for a new standard, either ratios or atom percents are entered; the program calculates the other.

\subsubsection{Atom percents}

The line labeled aAt Pcts, holds the isotopic masses of the element. In the line beneath is the isotopic composition in atom percent. These are the values listed on the output sheet when a sample identified as a routine standard has been analyzed. When entering information for a new standard, either ratios or atom percents are entered; the program calculates the other.

\subsubsection{Editing and inspecting a record}

Selecting 2 from the Stand menu allows you to inspect or edit an entry in the file. An example of the screen listing of the contents of a record is given in Table 3.12; the meanings of the various parameters are given in Section 3.8.2. The lower part of the display gives directions on how to proceed. If all you want to do is to inspect the record, entering a zero will send you back to the main File program menu. Entering 1 allows modification of the label; entering 2 or 3 allows masses or atom percents to be altered.

\subsubsection{Listing labels}

It is sometimes convenient to list the labels of the standards in the file. This provides a means of determining which standards are there and which records they are in. This list is available on either the screen or the printer. The contents of the file as of August 1992 are listed in Table 3.13. This is a file that changes very little with time. 
Table 3.13 List of STAND File Contents

\begin{tabular}{ccc} 
Record & Element & Label \\
\cline { 2 - 3 } & $\mathrm{U}$ & NBS U-0002 \\
2 & $\mathrm{U}$ & NBS U-005 \\
3 & $\mathrm{U}$ & NBS U-010 \\
4 & $\mathrm{U}$ & NBS U-015 \\
5 & $\mathrm{U}$ & NBS U-020 \\
6 & $\mathrm{U}$ & NBS U-030 -050 \\
7 & $\mathrm{U}$ & NBS U-100 \\
8 & $\mathrm{U}$ & NBS U-150 \\
9 & $\mathrm{U}$ & NBS U-200 \\
10 & $\mathrm{U}$ & NBS U-350 \\
11 & $\mathrm{U}$ & NBS U-500 \\
12 & $\mathrm{U}$ & NBS U-750 \\
13 & $\mathrm{U}$ & NBS U-800 \\
14 & $\mathrm{U}$ & NBS U-900 \\
15 & $\mathrm{U}$ & NBS U-930 \\
16 & $\mathrm{U}$ & NBS U-950 \\
17 & $\mathrm{U}$ & NBS U-970 \\
18 & $\mathrm{U}$ & NBL U-117 \\
19 & $\mathrm{U}$ & NBS Pu-946 \\
20 & $\mathrm{U}$ & NBS Pu-947 \\
21 & $\mathrm{Pu}$ & NBS Pu-948 \\
22 & $\mathrm{Pu}$ & NBL Pu-128 \\
23 & $\mathrm{Pu}$ &
\end{tabular}

The file includes all 19 of our NIST (NBS) uranium standards. The NIST identifying code is based on the atom percent $235-\mathrm{U}$ in the standard. They chose to multiply it by ten (for reasons unknown to me); thus U-200 is about $20 \% 235-\mathrm{U}$ and $\mathrm{U}-030$ is about $3 \%$. There is one important exception to this (again for unknown reasons): NIST U-950 is natural uranium $(0.7 \%)$ and not $95 \%$ 235-U. This is without question confusing, since U-9.30 and U970 are $93 \%$ and $97 \% 235-U$, respectively. NIST U-950 is important not only for being isotopically certified; it is also certified for uranium content and is our primary reference for calibration of spikes. Note that NIST U-950 has had several editions that are labeled U-950, U-950a, etc. We are now using U-950b.

Quality assurance people often want to see the certificates for the standards; these are available for all of the standards in the file.

\subsubsection{Printing the file}

You may get either the abbreviated list of labels (previous section) or the entire file listed on the printer or the screen. Selecting 3 or 4 from the Stand menu will result in your 
being asked for which one you wish to use. Selecting 4 gives you the entire file, including calibration ratios and isotopic composition.

3.8.6 Editing the last record used

This file uses a bookkeeping record in which is kept the value of the last record written to. Entry of a new standard will cause this value to be read, incremented by one, and assigned to the new entry. You should not have to worry about this situation; if the programmer did his job, it should require no attention. In case of trouble, it is almost certainly the programmer who will have to unscramble things.

\subsection{SAMP File}

Samp is far and away the file most frequently accessed using File. It contains the information required for correct processing of the data that are transferred to the MicroVAX from the PC. The information in it is either entered by the analyst during set-up or generated during the analysis. It is often desirable or necessary to reprocess data with one or more variables changed.

Selecting 7 from the File menu yields a window that looks rather like this:

Select editing and inspection index

0 to return to calling program

1 to list all variables

2 to list only 1 subset of variables

3 to change 1 variable only (no list)

4 to access bookkeeping record

These options are described below.

\subsubsection{Editing considerations}

The number of variables stored for each sample is too many to fit comfortably in one window. Instead, subsets of the variables are displayed on six successive windows, each of which allows editing of its contents. Short descriptions of these six windows are given in Table 3.14, and they are described in detail later in this section.

Table 3.14 Windows For Editing SAMP

$\frac{\text { Window }}{\text { Identification Info }}$
Calculation Parameters
File Record Info
Run Info
Spike Info
Subgroup Info

Description

Sample codes, etc.

Calculation index, etc.

File record numbers

First and last runs, etc.

Ratio, weights, etc.

Masses, sweep factors 
These are the six subsets of data that can be accessed by entering 2 at the Samp menu. Entering a 1 will result in all six screens being displayed in turn; bringing up the next window is effected by entering a 0 for the editing index. No changes need be made in any of the windows; entering a zero for the index choice moves the program forward without any changes being made in the data.

Some variables require changing more often than others. This is particularly true during debugging. Eleven variables can be altered by entering 3 for your menu selection. Entering the 3 results in a window that looks like the listing in Table 3.15.

\section{Table 3.15 Short Options Menu}

Select one parameter from the following:

0 to end alterations

1 to change calculation index (ICALC)

2 to reinitialize

3 to change Batch Code

4 to change masses in isotope dilution ratio

5 to change SPIKE file record no.

6 to change RATIO file record no.

7 to change 1 st run

8 to change last run

9 to interchange spike and sample

by new spike companion and new SPIKE file record

10 to change resin bead companion

11 to change original $\mathrm{Pu}$ date

Most of these should be self-explanatory. The reinitialize option (2) is described in Section 3.9.3.3. The variables are described in the next few sections, and variables are grouped according to the window from which they are addressed.

\subsubsection{Identification Info}

The data displayed and accessed in this window are shown in the example in Table 3.16 . 
Table 3.16 Identification Info Window

1. Element symbol

PB

2. Title

3. Unique sample id code

9761 PB-208

4. Resin bead companion id

$\mathrm{PB}(0) 25$

5. Unspiked sample id

6. Blank companion id

7. Contamination elements

8. Batch code

9. Analysis date

$2-5-92$

Each of these variables is described below. Each can be altered by entering the appropriate index at the prompt. To change the unspiked companion id, for example, enter a 5. The cursor will move to the appropriate place in the window and ask for an entry. Enter the new information (terminated by a "Return" or "Enter"); the cursor will then revert to asking for an index value. You may cycle through these steps as often as you like; editing will be terminated only when a value of zero is entered for the index.

\subsubsection{Element symbol}

This element symbol entry is important in that it is the one used to access mass and abundance information in the Massab file. The element symbols in the Spike and Ratio files, for example, are used only for identification purposes and do not directly affect the calculations. This one does.

\subsubsection{Title}

This is the descriptive title used to provide information about the sample. In the example above, the requisition number from the Stable Isotopes Section's request sheet is included. This is excellent practice: The customer's sample identification should be included in the title.

\subsubsection{Unique sample id code}

This is the six-character code that uniquely identifies the sample. It is used for searching the Out file in various routines in MSC. There is no restriction on which six ASCII characters are used. Some analysts like to include the instrument number and element symbol in the code, but this is merely a convenience and not a necessity.

What is important is that the six characters be unique to the sample in question. If the same code is used twice, both sets of results are written into the same record in the Out file; those from the last sample calculated will then be the only ones saved. 


\subsubsection{Resin bead companion id}

This is the unique six-character sample identification code for the companion sample in the analysis of mixed plutonium-uranium resin beads. In this type of analysis, both elements are analyzed from a single filament, and the 238 mass position must be corrected by subtracting $238-\mathrm{U}$ from $238-\mathrm{Pu}$ and vice versa. Note that the companions sample for uranium is plutonium and vice versa. Results for this companion sample must be in the Out file, and they are located by searching for this six-character code.

\subsubsection{Unspiked sample id}

This is the six-character code that identifies the unspiked companion sample for isotope dilution calculations. Results for this sample must be in the Out file and are located by searching the file for this six-character code. Naturally all six characters (including blanks) must be correct or the search will fail.

\subsubsection{Blank companion id}

This is the six-character code that identifies the blank when blank corrections are required. Information for this sample must be stored in the Out file.

\subsubsection{Contaminant elements}

This is an array holding up to three element symbols for contaminant elements. Each symbol occupies two characters, one of which may be blank. If a one-character contaminant symbol is included, it should be right-adjusted in the two-character field; that is, it should be (blank)U rather than $U$ (blank).

\subsubsection{Batch code}

The batch code is a two-character variable used to identify special calculational cases or to group samples from one project together. Full details of the batch code are given in Section 4.1.5. One important example is that, for reactor burn-up calculations, the batch code must be $B U$. This is to distinguish these samples from other neodymium and cerium samples for which burn-up calculations are not desired.

\subsubsection{Analysis date}

The values for the date are taken from the PC when the sample is run on the mass spectrometer. It is not necessarily the date on which calculations were made, although it usually will be because calculations are normally performed on the same date the data were taken. These data are of importance when the analyte element is radioactive and decays on a human time-scale. Most transuranium elements fit this description.

\subsubsection{Date to which to correct Pu isotopic}

This variable does not appear in Table 3.16. It will appear only when the element is $\mathrm{Pu}$. It is the date to which the isotopic composition of plutonium is to be corrected. Because 
of the relatively short half-lives of some plutonium isotopes (notably 241-Pu; half-life ca. 14.5 years), its isotopic composition changes fast enough to be significant in a few months. We have participated in many experiments where results from different laboratories were to be compared, and, for valid comparison of plutonium analyses, it was necessary that all results be for the same date.

A value for this date can be entered by the analyst at run time; it can also be entered here in File and the data reprocessed to give the desired results.

\subsubsection{Calculation Parameters}

This window lists the values of various indices that control the route taken by the calculations. An example of this window is shown in Table 3.17.

\section{Table 3.17 Calculation Parameters Window}

1. Calculation index (ICALC)

2. Mixture index

3. No. of passes through calculations

4. Blank index

These variables are described in the sections below.

\subsubsection{Calculation index}

This variable was named icalc in earlier versions of the programs--hence the last part of the label. It retains this name on the PC's and serves a different function there. It can have only two valid values, 0 and 1 . It is 0 until the data have been processed; after processing it takes on the value of 1 . This variable is used to identify which samples have not yet had their data processed when $M S C$ is first entered.

\subsubsection{Mixture index}

This variable is 0 for most samples and 1 for plutonium-uranium resin bead pairs. You should only rarely have to change its value, but you should be aware of the fact that, when its value is 1 , the program will be trying to treat the data as part of a pair of samples.

\subsubsection{No. of passes through calculations}

This variable is relevant only for plutonium-uranium resin bead pairs. These calculations require that the data for each element be processed twice: the first with no corrections made and the second with correction applied to the 238 mass position. The variable can thus have values of 0,1 , or 2 , depending on the status of calculations. 
You should not have to worry about changing this parameter explicitly from this screen. To reprocess the data for $\mathrm{Pu}-\mathrm{U}$ pairs, it is essential to reset this parameter to 0 . This is most easily accomplished by choosing reinitialize, from the Short Options menu ( 3 from the Samp menu and 2 from the Short Options menu). Making this choice will set both the calculation index and the number of passes to zero. This has the effect of fooling the program into thinking that the data have never been processed before. It is necessary in this case because the numbers of counts are changed in the Sumcts file during the second pass through the data to reflect correction for the other element of the pair. While this allows correct reprocessing of the data when treated as individual samples, it precludes correct calculations if you need to have both members of the pair recalculated.

\subsubsection{Blank index} otherwise.

An index that takes on a value of 1 when blank calculations are desired. It is 0

\subsubsection{File Record Info}

This window contains information relevant to various file record numbers. An example is given in Table 3.18 .

\section{Table 3.18 File Record Info Window}

1. Mass spec machine no.

2. RATIO file record no.

3. SPIKE rile record no.

4. Calibration index

5. STAND file record no. for calibration

6. CHDATA file record no.

Some of these variables should never need changing, but others are changed with some frequency. The variables are described below.

\subsubsection{Mass spec machine no.}

Each mass spectrometer is assigned an instrument code to facilitate calculations. The number is assigned by the $\mathrm{PC}$ with no input required of the analyst. Each instrument had its own unique dead time and bias stored in the Dtbias file. The Dtbias file is accessed by using the instrument number as the record number. It is thus highly unlikely that it will ever need to be changed.

Instrument numbers were assigned chronologically thirty years or more ago. Since some of the younger members of the staff haven't been privileged to work here that long, here is a table defining this variable. 
Table 3.19. Mass Spectrometer Instrument Numbers

\begin{tabular}{ll} 
Number & Instrument description \\
\hline 1 & Three-stage mass spectrometer \\
3 & Two-stage mass spectrometer in Back Lab \\
4 & Single-stage; no longer linked to the VAX. \\
5 & TRU mass spectrometer.
\end{tabular}

The single-stage mass spectrometer originally served by the VAX was moved some years ago to X-10, where it is now used in RIMS experiments. This number is thus available if a new instrument is acquired that needs to be linked to the VAX.

The VG-354 was at one time linked to the VAX; it no longer is, and considerable software development will be necessary to resurrect it. Plans are currently in the works to do precisely this.

\subsubsection{RATIO file record no.}

This is the record number in the Ratio file that determines which ratios are calculated. Altering this value will allow processing of the same data with different ratios being calculated.

\subsubsection{SPIKE file record no.}

This is the record number in the Spike file that contains the spike used in isotope dilution and internal calibration calculations. Changing this value allows calculation using a different spike, which should be necessary only if the wrong value was inserted originally.

\subsubsection{Calibration index}

This index is used only when an instrumental calibration standard is involved. It should be set to 0 if you don't want such calculations performed; it is 1 if you do.

\subsubsection{STAND file record no. for calibration}

This is the record number in the Stand file that holds the information for an isotopic standard. Unless the calibration index is 1 (see Section 3.9.4.4), calculations follow the normal route. This record is read and the isotopic composition of the standard listed on the second output sheet. This is for comparison purposes only, to allow you to compare the results of the given analysis with the certified values. 
3.9.4.6 CHDATA file record no.

This is the starting record in the Chdata for the channel-by-channel raw counts. They are transferred from the PC to the VAX. Note that only the raw counts and the information in the Samp file are so transferred. The other files all reside permanently on the VAX.

There is one record in Chdata for each eight subgroups of data. For most samples, then, there will be one record used per run. When more than eight subgroups are used, two Chdata records will be used per run, the first for subgroups $1-8$, the second for subgroups $9-n$ (maximum 16).

There is no reason to change this value. It is displayed for informational purposes in the event of trouble. Changing it will cause far-reaching ramifications, requiring changing several other parameters as well. Leave it alone.

\subsubsection{SUMCTS file record no.}

This is the record number in the Sumcts file in which the condensed raw counts start for the sample in question. Each run requires a separate record, so the number of records will equal the number of runs. It is the condensed raw data in Sumcts that are accessed when data are processed more than once and which are listed on the first page of output in MSC.

Like the similar variable associated with the Chdata file, there is no reason to change this variable. It is displayed for information only.

\subsubsection{Run Info}

The Run Info window holds the values of parameters associated with the length of the analysis. An example is given in Table 3.20.

\section{Table 3.20 Run Info Window}

1. No. of last run

2. No. of first run

3. No. of nighest subgroup

1

4. No. of cycles/run

5. Sweep rate (sec/chan)

These variables are described below. 


\subsubsection{No. of last run}

This is the number of the last run to be processed and not necessarily the number of runs actually recorded. For example, if you take 16 runs and the first five are to be rejected. the number of the last run is 16 , but the number of runs processed is 11 . On the other hand, if you take 16 runs and the last six are bad, you can replace the 16 with 10 and have the six runs ignored by the calculations.

The transfer program (Chapter 7) will transfer all raw data from the PC's to the VAX no matter what values are given to the last and first runs. This is to allow the analyst access to all data for calculations using the VAX.

\subsubsection{No. of first run}

This is the number of the first run whose data are to be included in the calculations. The most common runs to be rejected occur either at the start or near the end of the analysis. By changing the value of the first run from, say, 1 to 5 , it is easy to eliminate the first four runs from the calculations. You could always specify each run individually from program MSC (Section 5.1.3), but that is more cumbersome than simply changing the value of the first run through program File.

\subsubsection{No. of the highest subgroup}

It is important to understand that this variable is not necessarily the number of mass positions monitored. It often will be, but it is not necessary. The value of the variable here is the number of the highest subgroup used. If you have peak in the eighth subgroup, for example, and subgroups $2-5$ have not been used, the value here will be 8 , not 4 . The value of this variable is set by the software on the PC; it should not be changed in this program except for debugging purposes.

\subsubsection{No. of cycles/run}

This is the number of times the program on the PC has cycled through the spectrum during one run. This number is set by the corresponding value in the Scan file on the PC. It is usually calculated to make the time for a run about 9() seconds. It should not be changed.

\subsubsection{Sweep rate (sec/chan)}

This is the length of time counts are registered for each channel. It is virtually always $2 \mathrm{msec}(0.002 \mathrm{sec})$, although this is not mandatory. It is read from the switch setting on the Sweep Control Panel during data-taking. It should not be changed.

\subsubsection{Spike Info}

This window holds information relevant to the spike. It will of course have no bearing on normal isotopic analyses; only when isotope dilution or internal calibration calculations are called for is it relevant. An example of this window is given in Table 3.21. 
Table 3.21 Spike Info Window

1. SPIKE file record no.

2. Isotope dilution ratio

3. Reverse iso diln index

4. Spike weight

5. Sample weight

6. Dilution weight

7. Aliquot weight

8. Density
5

$0 / 0$

0

$0.1000 \mathrm{E}+01$

$0.1000 \mathrm{E}+01$

$0.1000 \mathrm{E}+01$

$0.1000 \mathrm{E}+01$

$0.1000 \mathrm{E}+01$

\subsubsection{SPIKE file record no.}

This is the record number in the Spike file that holds isotopic and other information for the spike used. This is the same value that can be accessed from the File Record Info window (See Section 3.9.4.3). Changing its value allows you to calculate the data using a different spike.

\subsubsection{Isotope dilution ratio}

This is the isotope ratio to be used in isotope dilution calculations. It takes on the value in the Spike file by default (when the values in the Samp file are 0) but can also be specified here. It can also be entered at run time in MSR by the analyst at the PC. Non-zero values override those in the Spike file.

An example of when a different value for this ratio is desirable is if you are using a uranium spike whose information is already in the Spike file for an isotope dilution analysis of a highly enriched sample. The isotope dilution ratio in the Spike file is probably 238/233, and you will want to change it to $235 / 233$ for this and similar samples. You can either do so by entering the new value in the Samp file or by changing the ratio in the Spike file. If you choose the latter, you should remember to change it back when analysis of the enriched samples is complete.

\subsubsection{Reverse iso diln ratio}

This variable is set to 1 when you want to perform a "reverse" isotope dilution analysis; it will be 0 otherwise. You will most often want to do this when you are calibrating the concentration of a spike against a quantitative standard.

This variable is provided for convenience; it swaps the sample and spike information for the calculations. The spike being calibrated (actually the sample in a reverse isotope dilution analysis) is probably stored in the Spike file; the sample being used for the calibration (the spike in reverse isotope dilution) is often natural uranium whose isotopic composition is kept in the Out file. You avoid the tedium of entering information in both files by using a value of 1 for the reverse isotope dilution index. 


\subsubsection{Spike weight}

This is the weight of the spike used in an isotope dilution analysis. You should be aware that the units in which you enter this variable will be the units of the numerator of your concentration. If you enter your spike weight in micrograms, your concentration will be in units of micrograms per something. Units for the denominator are defined by the sample weight, as described in the next section.

\subsubsection{Sample weight}

This is the weight of the sample used in an isotope dilution analysis. Note that the units of this weight will be those of the denominator of your concentration. If you enter this value in grams, your concentration will be in units of something per gram. See Section 3.9.6.5 (Spike weight) for the numerator units.

\subsubsection{Dilution weight}

This is the weight of the volume to which the sample was diluted if it in fact was. A value of 1.00 is used when no dilution was made.

\subsubsection{Aliquot weight}

This is the weight of the aliquot drawn from the dilution which is represented by the dilution weight. If no dilution was made, this variable is set to 1.00 .

\subsubsection{Dilution factor}

This variable is calculated in the program and not entered; it thus does not appear in Table 3.21. It is calculated from dilution, sample, and aliquot weights according to the following equation.

$$
\mathrm{df}=\mathrm{Wd} /(\mathrm{Ws} * \mathrm{Wa})
$$

where:

$$
\begin{aligned}
& \mathrm{df}=\text { dilution factor } \\
& \mathrm{Wd}=\text { dilution weight } \\
& \mathrm{Ws}=\text { sample weight } \\
& \mathrm{Wa}=\text { aliquot weight }
\end{aligned}
$$

Note that you will want your dilution and aliquot weights to be the same units so that the dilution factor will have units of the reciprocal of the sample weight.

\subsubsection{Density}

This is the density of the sample solution and allows calculation of concentration on a volume basis in addition to the normal weight basis. Volume calculations are fraught with uncertainty because a solution's density is a function of the temperature. Weight is a more reliable unit to use, but some customers insist on volume-based concentrations. The inclusion of density caters to their desires. If no density is entered, it is assumed to be 1.0, and 
concentrations calculated using it will be numerically equal to those calculated on a weight basis.

\subsubsection{Subgroup Info}

This window lists masses and sweep factors; an example is shown in Table 3.22.

Table 3.22 Subgroup Info Window

$\begin{array}{lrcccccccccccccrr}\text { Subgroup } & 1 & 2 & 3 & 4 & 5 & 6 & 7 & 8 & 9 & 10 & 11 & 12 & 13 & 14 & 15 & 16 \\ \text { 1. Masses } & 35 & 204 & 206 & 207 & 208 & 0 & 0 & 0 & 0 & 0 & 0 & 0 & 0 & 0 & 0 & 0 \\ \text { 2. Sweep } & 1 & 4 & 1 & 1 & 1 & 0 & 0 & 0 & 0 & 0 & 0 & 0 & 0 & 0 & 0 & 0\end{array}$

These variables are set in the Scan file on the PC and should rarely, if ever, be changed.

\subsubsection{Masses}

The mass number associated with the indicated subgroups. Remember that the background mass number (subgroup 1 in Table 3.22) must differ by at least 10 from the base mass for bias correction (208 here). It must also have a value greater than 20 . The value in the table (35) is an abbreviation of 203.5, but this convention isn't necessary; any number differing from 208 by more than 10 would have worked just as well. The convention has the advantage of reminding you of where you set the background subgroup, however.

\subsubsection{Sweep factors}

The sweep factor is the number of times the given subgroup was swept for each traversal of the mass spectrum. The number of counts in each mass position is divided by its sweep factor before ratios are calculated.

\subsection{Raw Data Files}

There are two raw data files, Chdata (for channel data) and Sumcts (for Sum of Counts). The former holds the channel-by-channel raw data for the sample; there are 32 real numbers per subgroup. It is transferred from the PC to the VAX when program Tran (Chapter 7) is executed. Sumcts is a compressed version of Chdata, with the number of data points per subgroup reduced from 32 to one.

You should very rarely have to access either of these files; the possibility is offered only as a trouble-shooting aid. Raw data should never be changed.

Access to these files is effected by entering 8 as you selection from the main File menu. A second menu appears in its own window and looks like this: 
Select file:

0 to return to calling program

1 for sum of counts file

2 for single-channel data

3.10.1 The Sumcts file

Table 3.23 is an example of the window that presents Sumcts data.

\section{Table 3.23 Sumcts Window}

Record no.

1. Element symbol

2. Sample id code

3. No. of channels in sum

Indx

4

5

6

7

8

9

10

11
Counts

320000

80000

640000

320000

640000

320000

80000

80000
777

U

Test

32

Indx Counts

120

130

140

150

160

$17 \quad 0$

180

190

Editing is effected in the normal way by entering an index value and then the new value for the variable. The example in Table 3.23 illustrates one of the very few legitimate reasons to enter data in this way. This is an artificial spectrum that the programmer entered to aid in debugging. There are no conditions under which raw data for a real sample should be altered.

\subsubsection{The Chdata file}

Table 3.24 gives an example of the list generated from the Chdata file. Each subgroup requires four lines at eight channels per line, and only two subgroups of the spectrum are given in the example. It is outstandingly tedious to type in the numbers, and the example should suffice to give you an understanding of the presentation. At the terminal, two windows are needed for each eight-subgroup run. The display for the first four subgroups is held until you hit a key to proceed, whereupon the second set of four subgroups is shown. Analyses using more than eight subgroups will take four windows for complete presentation of the data.

Access is given to these data for inspection purposes only. On no account are any channel count values to be changed. It can be helptul in trouble-shooting to be able to inspect individual channels. You may want to find out if a suspiciously high background count 
is due to a noise spike or to a generally high number of counts in all channels. Looking to see if one or two channels contain the lion's share of the counts (indivating a noise spike) is a good way to do this, as indicated in the value of 5000 listed in Table 3.24 in a subgroup whose counts are otherwise 0 .

Table 3.24 CHDATA File Window

Record no. $\quad 888$

$\begin{array}{rrrrrrrr}6770 . & 6699 . & 6811 . & 6733 . & 6893 . & 6744 . & 6656 . & 6585 . \\ 6715 . & 6880 . & 6617 . & 6620 . & 6643 . & 6480 . & 6626 . & 6813 . \\ 6588 . & 6715 . & 6478 . & 6514 . & 6565 . & 6809 . & 6737 . & 6643 . \\ 6527 . & 6739 . & 6693 . & 6608 . & 6710 . & 6554 . & 6684 . & 6709 . \\ 0 . & 0 . & 0 . & 0 . & 0 . & 0 . & 0 . & 0 . \\ 0 . & 0 . & 0 . & 0 . & 0 . & 0 . & 0 . & 0 . \\ 0 . & 0 . & 0 . & 0 . & 5000 . & 0 . & 0 . & 0 . \\ 0 . & 0 . & 0 . & 0 . & 0 . & 0 . & 0 . & 0 .\end{array}$

\subsection{Out File}

The Out file stores results of calculations. There is room for output from 3,000 samples before data are written over. Results stored here are used in isotope dilution and mixed resin bead calculations; they are also used by program Rep (Chapter 6) to generate a summary report.

Out can be accessed either from program File or program Rep. From program File, it is reached by entering 9 for the menu selection from the main menu. Doing so gives a new menu window that looks sort of like this:

Select editing index

0 to RETURN to calling program

1 to edit or inspect a record

2 to enter companion info

3 to enter PERMANENT companion info

4 to list permanent companions

5 to correct NBS-947 to any date

6 to change the last record used in Out file

7 to shift data to a new record

8 to access BKOUT (bookkeeping for Out)

These options are described below. 
3.11.1 Editing and inspecting a record

It is sometimes helpful in trouble-shooting to be able to inspect a record in Out. An example of so doing is given in Table 3.25.

\section{Table 3.25 OUT File Contents}

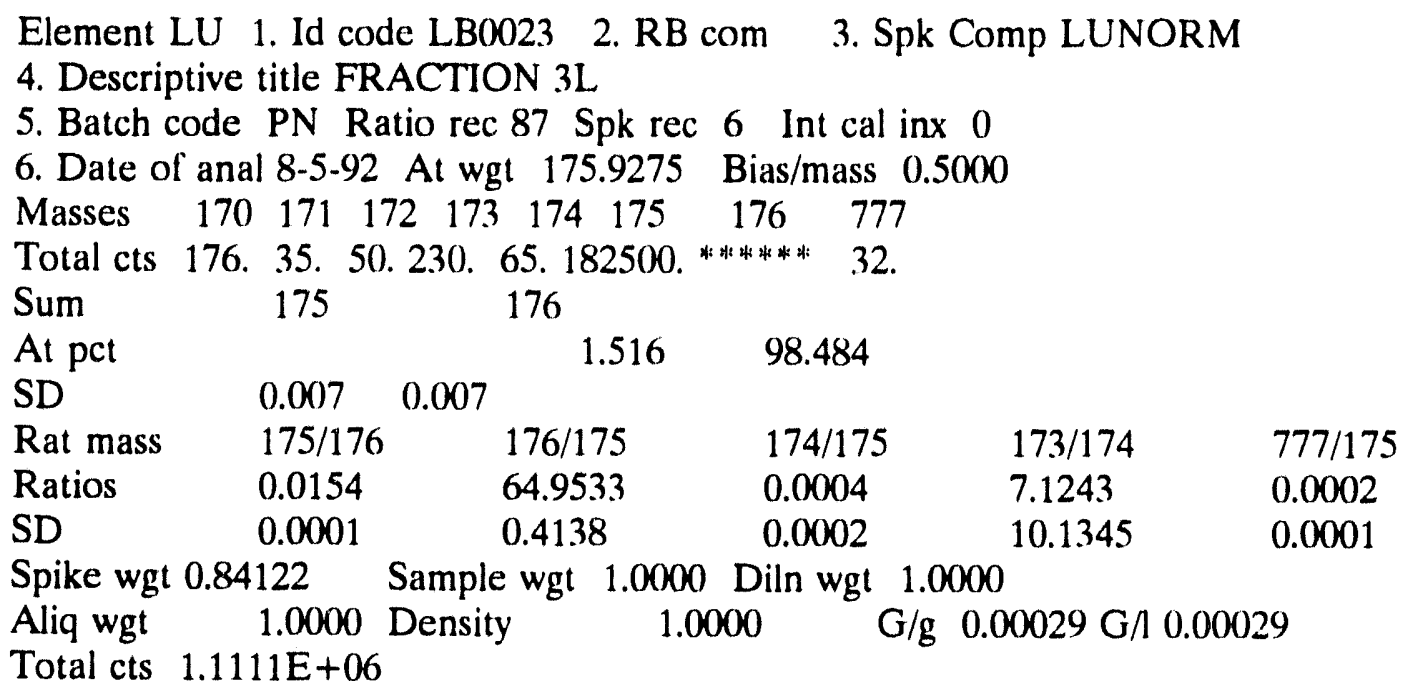

It clearly would be flagrant cheating to alter a calculated value in a record like the one in Table 3.25. Only those values with index numbers (1-6) can be changed. None of these affect the mathematical results. The asterisks listed for the number of counts of 176 means the value is too big for the field specified by the Fortran program.

This is admittedly a cluttered presentation. Access to the Out file for this reason is needed so seldom that it was deemed not worth the time to make it spiffier. It would require a multi-window display, for one thing.

\subsubsection{Entering companion info}

This option allows speedy entry of the information needed for use as a companion; companions are used in isotope dilution and mixed resin bead calculations. A window opens and prompts you to for input. Only the minimum amount of information is requested. Following the instructions should be a piece of cake. Entry occurs in the next available record unless you specify otherwise.

\subsubsection{Entering PERMANENT companion info}

The first 50 records of Out are reserved for what are called "permanent" companions. These are samples repeatedly used as companions, especially in isotope dilution calculations. 
Natural uranium is an example of a permanent companion. LUNORM, the unspiked companion in the example above, is natural lutetium and is another example. NBS Pu-947 is also there.

Entry is to the next available record among the first 50; if these are all full, you are warned and allowed to choose where to store the new entry.

\subsubsection{Listing permanent companions}

This option provides a list of the permanent companions in the first 50 records of Out. You may list either to the terminal or to the printer.

\subsubsection{Correcting NBS Pu-947 to any date}

Plutonium has isotopes that decay fast enough to alter the isotopic composition of the element on a human time scale. The isotope most affected is 241-Pu, which has a half-life of about 14.5 years. Even a few months is enough to make an observable difference. The standard was certified as of October 13,1971, as being $4.540 \%$ abundant: this number had decreased to about $1.75 \%$ in February 1992.

This option alluws you to adjust the composition of NBS Pu-947 (stored in the permanent companion section of the file) to whatever date you choose. This is often helpful for comparison purposes when a spike is being calibrated in an interlaboratory round-robin.

\subsubsection{Changing the last record in Out}

You should not have to fool with this; it is the responsibility of the programmer. Entries to Out, like several other files, are controlled by keeping track of the last record used. When a new entry is called for, this value is read from the bookkeeping record, incremented by one, and the new data assigned to it. It is supposed to be self-policing; since it's been several years since the programmer (me) has had to worry about it, it's improbable it'll give trouble in the future.

\subsubsection{Shifting data to a new record}

This used to be more useful than it is today. The old Out file, due to the limited size of the hard drives of the day, was much smaller than it is now. There were occasions when records in the Out file scheduled for near-term use contained results that people wanted retained in the file. One solution was to write a small routine that shifted the treasured results to new records. The only input required is for the source and destination records. It should be easy.

\subsubsection{Accessing BKOUT}

Bkout is the bookkeeping file for Out. It was created when Out became so large that searching for an entry on the basis of the six-character sample code took too much time (45 seconds is a very long time, indeed, on occasion!). The sole contents of Bkout are the sixcharacter codes for each record. They are stored sequentially in the Bkout file, so their 
location in the sequence corresponds to the record number they occupy in Out. This is the programmer's territory. You should never have to worry about this option. 


\section{PROGRAM MSR}

MSR is the program that controls data acquisition. It resides only on the PC's: a VAX version would make no sense as the VAX has no means of communicating with data-taking hardware. MSR is organized into two main subroutines: one is called Setup and is the one through which the analyst inputs information for the sample at hand; the second is called Msop (for mass spec operate) and controls data acquisition. The operations necessary for taking data are described in this chapter.

\subsection{Input of information}

Normal routine calls for the computer and mass spectrometer to be turned on in rapid succession when preparing to take data. Once the computer is booted, entering $M S R$ from the terminal begins execution of the program. Table 4.1 shows an example of the input window for $M S R$. The analyst enters data; the amount required depends on the type of analysis to be performed.

Table 4.1 Set-Up Window

Input Parameters

1. SAMP file record no.

12

2. SCAN file record no.

3. Sample id.

4. Descriptive title

U10039

5. Batch Code

6. RATIO file record no.

U23.3 SPIKE

7. Resin bead companion id.

8. SPIKE file record no.

1

9. Unspiked companion id.

10. Isotope dilution ratio

11. Spike weight

0

12. Sample weight

1.0

13. Dilution weight

1.0

14. Aliquot weight

1.0

15. Density

1.0

1.0

16. Reverse iso diln index 17. Contaminant el symbols Blank companion id

Index no. to correct, 0 to proceed:

Each of the variables for which input is requested is described in Section 3.9. Only short descriptions will be given here. 
Note that the minimum amount of information you must input for an analysis is the Scan file record number (have to know how to scan!), the sample identification code (for use in Out), and the Ratio file record number (have to know which ratios to calculate). None of the others is required, although you'd better have a Spike file record number if you're doing an isotope dilution analysis.

An area near the bottom of the screen is used to display instructions or information. The contents of the Scan file record chosen are displayed here, for example.

4.1.1 SAl. $\quad$ record no.

This recuid number is assigned by the PC and not entered by the analyst. It is important to distinguish between the Samp file record used on the PC and that used on the VAX. There will be a different record number on each computer for every sample. It is not possible to have the same number used on each computer because three instruments feed data to the VAX. There is room for 20 samples on each PC and for 400 on the VAX.

The calculation index (also called icalc) is set to 0 on the PC before the data for the sample have been sent to the VAX; it is 1 afterwards. On the VAX, the value is 0 until the data have been processed and 1 thereafter. index.

See Section 3.9.3.1 for more information about the VAX version of the calculation

\subsubsection{SCAN file record no.}

This is the record in the Scan file in which the parameters governing the acquisition of data are stored. An example of Scan file record is given in Section 3.4.2. Once the record number is entered in $M S R$, the contents of the record are displayed at the bottom of the screen to allow inspection; no option is provided for changing the Scan file from MSR. If the Scan file you get isn't what you want, you need to leave MSR (hit CTRL/C) and either enter a different Scan file record number or edit the Scan file to meet your needs.

\subsubsection{Sample id}

This is the six-character code that uniquely identifies this sample for the computer. Section 3.9.2.3 describes its use in detail. Any combination of six ASCII characters can be used; the only important thing to remember is that they must be unique for each sample.

\subsubsection{Descriptive title}

This variable accommodates up to 20 characters; the purpose is to identify the sample from the laboratory point of view. This is in contrast to the sample identification code, which identifies the sample for the computer. It is excellent practice to include the customer's sample identification in this variable. 


\subsubsection{Batch code}

The batch code is described in detail in Sections 3.9.2.8 and 5.2.2.9. It is used as a means of identifying samples that require special calculations performed; reactor burn-up calculations based on neodymium. for example, require the batch code BU. It can also be used to identify a group of samples that form a single project. Plogram Rep (Section 6.7) uses it to list only the samples of interest.

\subsubsection{RATIO file record no.}

Like the sample identification code, you must enter a value for the Ratio file record number. No calculations can be performed without one, and experience has shown it is less hassle to enter a Ratio record number when one is not needed than is caused when a needed value is omitted. MSC skips the sample in question if a 0 value for the Ratio record is encountered.

Details of the Ratio file are given in Section 3.5.

\subsubsection{Resin bead companion id}

This is the six-character code for the companion sample in mixed plutonium-uranium resin bead analysis. Details of this process are given in Section 5.2.2.5 and 3.9.2.4.

This is the code entered as the unique sample identification code when the companion was itself analyzed. File Out is searched for this set of six characters. Note that plutonium is the companion sample for a uranium analysis, and uranium the companion for a plutonium analysis. In a similar way, it is used in neodymium burn-up analyses. In this case, cerium is the companion for neodymium and vice-versa.

\subsubsection{SPIKE file record no.}

Index numbers 8-15 deal with spike information in an isotope dilution analysis. They can be ignored for other analyses. If a 0 is entered for the Spike file record number, all the remaining items are skipped and the correction index called for. Section 3.7.

The Spike tile record number identifies which spike was used. It is described in

\subsubsection{Unspiked companion id}

Like the resin bead companion, this is the six-character code the unspiked sample was given when it was analyzed. Note that, in this case, the companion of a uranium isotope dilution analysis will be another uranium sample; contrast this with the situation for a mixed resin bead analysis. 


\subsubsection{Isotope dilution ratio}

This is the identity of the ratio in mass numbers that is to be used in calculating the concentration in isotope dilution calculations. If you wish to use the value for this ratio stored in the Spike file (which will usually be the case), leave the entries 0 . This tells the program to use the default values. If, however, you want a different ratio used, enter the values in 213 fields. 213 is a Fortran field specification that tells the computer how many characters to expect (.3) and what kind of variable it is (I for integer in this case). The 2 tells it that two such variables will be entered. As an example, suppose you were analyzing enriched uranium and wanted the 235-U/233-U ratio to be used for calculating concentrations rather than the default 238-U/233-U. You would enter 235233; no spaces, commas, or other characters. For copper, say, you would enter 063065; each number must occupy three spaces. You could also enter 6365 for copper, using spaces instead of 0 's.

This variable is also described in Section 3.7.2.5 and 3.9.6.2.

\subsubsection{Spike weight}

This is the weight of the spike added to the sample for an isotope dilution analysis. Concentrations will be calculated with the units of the spike weight in the numerator; that is, if you enter the spike weight in micrograms, the concentration will have units of micrograms per something. The units of "something" are defined by the sample weight.

See Section 3.9.6.4 for more details.

\subsubsection{Sample weight}

This is the weight of the sample to which the spike was added. The concentration calculated will have its units in the denominator; if the sample weight is entered in grams, concentration will be in units of something per gram. The "something" will be the units used for the spike weight.

See Section 3.9.6.5 for more details.

\subsubsection{Dilution weight}

The weight of the dilution sometimes made for isotope dilution analyses. It defaults to 1.0 ; a 0.0 value would cause problems in the calculation of concentration. It should have the same units as aliquot. See Section 3.9.6.6 for more information.

\subsubsection{Aliquot weight}

The is the weight of the aliquot drawn from the dilution for some isotope dilution analyses. It should have the same units as the dilution weight. If you make no entry here, a value of 1.0 will be assigned to allow proper calculation of concentrations. Aliquot weight is discussed further in Section 3.9.6.7. 


\subsubsection{Density}

An entry is needed for density only if you want concentrations in terms of volume rather than weight. Volume is not as reliable a measure of concentration as weight because density varies with temperature and is difficult to measure accurately. Some customers, however, insist on concentrations being reported on the basis of volume.

If you have no need of a volume-based concentration, the value of the density will default to 1.0 ; concentrations calculated using it will then be numerically equal to concentrations based on weight. See Section 3.9.6.9 for more information.

\subsubsection{Reverse isotope dilution index}

This variable is one of three that is rarely used; to avoid the inconvenience of making an entry every time information is entered for a sample, the last three variables are accessed by entering the appropriate index number when all other input is complete (Section 4.1.19). This variable is accessed by entering 16 , contaminant element symbols by 17 (next section), and a blank companion by 18 (Section 4.1.18).

The reverse isotope index is 0 for all analyses except for cases of (naturally) reverse isotope dilution. Setting this variable to 1 causes program $M S C$ to swap spike and sample. This is a convenience when a material that is to be used as a spike is being calibrated against a gravimetric standard such as NIST U-950.

See Section 3.9.6.3 for more details. Don't forget that, if you forget to enter the 1 when entering data originally, you can always do it later at the VAX through program File.

\subsubsection{Contaminant element symbols}

As many as three contaminant elements can be corrected for. This variable holds their symbols in $3 \mathrm{~A} 2$ fields. This means that each symbol must be two characters wide (the 2 ), that any ASCII character is valid (the A), and that there are three symbols possible (the 3 ). Thus, to enter molybdenum and ruthenium, your input be MORU, with no spaces or punctuation between the element symbols.

Information for the element symbol you use must be in the Massab file. See Section 3.9.2.7 for more information.

\subsubsection{Blank companion id}

This is the six-character sample code that identifies the blank when such a correction is to be made. The blank must be in the Out file. See Section 3.9.2.6 for more details.

\subsubsection{Index no. to correct, 0 to proceed}

This index is the mechanism that allows you to correct erroneous entries before proceeding with the analysis. The value you want to enter is indicated by the index number associated with the variable. You would, for example, enter a 6 (followed by a return (or 
enter)) in response to the screen shown in Table 4.1 if you wanted to change to value for the Ratio file record number. The 6 being entered, the cursor shifts to the appropriate place in the input window and accepts the new value for the Ratio file record number. It then returns to the bottom of the window to ask for another index. You can change as many variables in this manner as you wish; the program will cycle from asking for an index value to asking for a new variable value until you enter a 0 for the index. This indicates to the program that all input is correct and that it may proceed with the analysis.

Of course, being excellent typists, you will not often have to correct anything. Entering a 0 for the index at the first opportunity moves the program forward.

The last three variables listed in the input window $(16,17,18)$ can be entered only by using this little procedure. If the sample to be analyzed is a reverse isotope dilution one, enter 16 for the index and then 1 for the value of the reverse isotope dilution index. Similarly, enter 17 and then the element symbols to correct for contaminants. Entering 18 and then the sample code for the blank gives access to blank correction calculations.

\subsection{Taking data}

Once a 0 is entered for the correction index, the instrument is ready to accept ion counts. The Sweep Control Panel has to be in "Wait" before it will let you proceed; an error message occurs if it isn't.

Operating the instrument is covered in a previous publication ${ }^{9}$ and will not be dealt with explicitly here. A short summary of instrument operation is given in Chapter 10 .

Once you have the ion beam properly focused and are ready to take data, set the Sweep Control Panel to the data acquisition condition. A running summary is displayed on the terminal during the analysis. An example is shown in Table 4.2

Table 4.2 Analysis Summary

Sample U10039 No. Sg 8 No. Cycles 80 SAMP File 12 CHDATA File 653

$\begin{array}{lcrrrrrrr}\text { Subgroups } & 1 & 2 & 3 & 4 & 5 & 6 & 7 & 8 \\ \text { Masses } & 315 & 233 & 234 & 235 & 236 & 365 & 238 & 239 \\ \text { Sweep Factors } & 4 & 1 & 4 & 4 & 4 & 0 & 4 & 1 \\ \text { Run } & \text { CR 233. } & 235 / 238 & & 238 / 233 & & 234 / 235 & \\ 1 & 5.54 \mathrm{E}+05 & .014344 & & .000212 & .714286 & \\ 2 & 3.09 \mathrm{E}+05 & .009597 & & .000203 & .800000 & \\ 3 & 3.17 \mathrm{E}+05 & .017730 & & .000214 & .550000 & \end{array}$

The meanings of the various parameters are given below. 


\subsubsection{Sample}

This is the unique six-character sample code entered in the set-up routine.

\subsubsection{No. Sg}

This is number of the highest subgroup as specified in the Scan file.

\subsubsection{No. Cycles}

This is the number of cycles covering the mass spectrum that comprise one run; it is specified in the Scan file.

\subsubsection{SAMP File}

This is the Samp file record number. It is assigned by the software and not entered by the analyst.

\subsubsection{CHDATA File}

This is the record number in the Chdata file in which data for the first run are stored. Subsequent runs are stored in the next records in the file; the record number increments by one or two after each run depending on how many subgroups are being swept. For eight or fewer, it increments by one; for more than eight, it increments by two.

This parameter, like the Samp file record number, is assigned by the software and not entered by the analyst.

\subsubsection{Subgroups}

This is just a counter to help correlate the information below with the correct subgroups.

\subsubsection{Masses}

The isotopic masses as read from the Scan file. Note that 315 (for 231.5) is the background position. Mass 365 is a residue from the days when we had to correct for scatter of mass 238 into mass 236 ; it stands for 236.5 mass position. This is no longer necessary, and I can see no reason for it be included. Mass 239 is swept to monitor plutonium. This is good practice; it is all too easy to get samples contaminated, and this is a good check on whether that has happened for the sample under analysis.

\subsubsection{Sweep Factors}

These are the sweep factors as specified in the Scan file. For this sample, an enriched 233-U spike, all minor uranium isotopes are swept four times for each time the major (233) is swept; the background is also swept four times. Increasing the number of sweeps per run for low-abundance isotopes improves the statistics for their measurement. 


\subsubsection{Run}

This is simply the run number and is incremented after the data for each run are stored.

\subsubsection{CR 233}

This is the rate in counts per second at which the most abundant isotope is being counted. Which isotope is used for this purpose is not specified and is undefined until after the first run has been taken. At that time, the program identifies which mass position has the most counts and calculates the count rate associated with it. This means that this variable is undefined until that point; the value listed during the first run has no meaning. To avoid confusion, the most intense peak in the first run is assumed to be the most intense in all subsequent ones. The only time this wouldn't be true would be if a contaminant started at an intensity higher than that of the major sample isotope. In that case, it is possible for the contaminant to decay during the course of the analysis to the point where it is less intense than the sample peak. This has been observed, for example, when running mixed plutoniumuranium resin bead samples. Occasionally, there is too much plutonium on the bead, making it difficult or impossible to burn it off before initiating data acquisition for uranium. The 239. $\mathrm{Pu}^{+}$can be more intense than the $238-\mathrm{U}^{+}$and decay well below it during the analysis. Since the isotope associated with the count rate is displayed on the terminal (after the first run), the analyst should be aware of what has happened and not be too concerned.

Count rates are displayed in $E$ fields in Fortran terminology. The ' $E$ ' stands for exponent, and the number after the ' $E$ ' in the output field is an exponent. Thus, the first count rate listed is $5.54 \mathrm{E}+05$ and should be read as $5.54 \times 10^{5}$ or 554,000 counts per second.

Monitoring the count rate is a good way to get a feel for the quality of the analysis. If it changes too much, you should refocus and perhaps adjust the temperature.

\subsubsection{Display Ratios}

As many as three ratios can be displayed on the terminal during an analysis. In the example in Table 4.2, those ratios are 235/238, 238/233, and 234/235. These ratios are specified in the Scan file and should be chosen to provide the best diagnosis of the analysis. Default values (Section 3.4.2.4) can also be used; in this case, ratios have 0's for numerators and denominators until after the first run. Monitoring these ratios for abrupt changes of value (after refocusing, for example) or for a monotonic increase or decrease (indicating isotopic fractionation or trouble with a contaminant) is good practice.

\subsubsection{After an analysis is complete}

When you press the "End Sample" button the Sweep Control Panel, data-taking stops. A message appears below the table of count rates and ratios telling you what has happened and how many runs were taken. 


\subsubsection{Temp and comments}

This variable is used primarily to indicate the temperature range over which data were taken. Although it is not necessary to enter the temperature, it is good practice to do so because, in case of questions, that information is then readily available. Any comments you wish to make ("Poor run," for example) can be appended to the temperature. It might look something like this: $1800-1910$ Poor run.

\subsubsection{Starting run}

This is the run at which you want processing of data to begin. It is a fairly frequent occurrence for a sample to take a few runs to stabilize, either with regard to count rate or to ratio value. This option provides a convenient means of eliminating the unwanted data.

Not entering a value (by hitting return) has the same effect as entering 1.

\subsubsection{Ending run}

This is the run at which you want data processing to stop. Another relatively frequent occurrence is to take data beyond the point where the sample is capable of giving good results. This provides an easy way to eliminate unwanted runs.

Not entering a value by hitting Return or Enter is the same as saying that you want the last run taken to be used for this value.

Note that, no matter which values you enter for starting and ending runs, all data collected are sent to the VAX. Thus, if someone wants to see all data processed, it is only necessary to make the required changes in the Samp file (using program File) and recalculate.

\subsubsection{Sweep rate}

The sweep rate is not explicitly listed on the terminal but is needed for the calculations. It is read from the switch setting on the Sweep Control Panel and recorded in the Samp file. Software prevents changing this value during an analysis. If you inadvertently start at the wrong setting, you will be informed through a message on the terminal. Set the sweep rate selector switch to its correct value and hit "Enter" to proceed with the analysis. 


\title{
5. PROGRAM MSC
}

MSC is the program that processes the data collected by program MSR (Chapter 4) and sent to the VAX by program Tran (Chapter 7). Although the program itself is logically complex and a lot goes on, it is quite simple to use from the analyst's point of view. It is essential that all files used to guide the calculations be properly set up before invoking MSC, and it is here that confusion can occur. Setting up the files is described in Chapter 3.

\subsection{Execution of MSC} terminal:

Upon entering R MSC on the VAX terminal, you get the following display on the

Table 5.1 MSC Menu

\author{
Program branch options \\ 0 for normal calculations \\ 1 to recalculate \\ 2 to reject runs \\ 4 for individual channel list \\ Sum for any combination: \\ 3 for recalc \& reject \\ 5 for recalc \& list \\ 6 for reject \& list \\ 7 for all 3 \\ 9 to list SAMP.DAT contents \\ 10 to select output device
}

The various options are explained below.

\subsubsection{Option 0 (normal calculations)}

If you choose 0 for your $M S C$ option, no further input is required--or allowed, for that matter. The program searches the Samp file for samples whose calculation index is 0, puts them in a queue, and calculates them in the sequence in which it found them. There are a few conditions where this sequence is altered, notably for mixed plutonium-uranium analyses where the data for each sample must be processed twice. This situation is described in detail in Section 11.6.

If no samples with calculation indices of 0 are found, a message to this effect is written to the terminal, $M S C$ is exited, and control returns to the monitor. 


\subsubsection{Option 1 (recalculate)}

This is the option to choose if you want the data reprocessed for any reason. There are several relatively common reasons to want to do this. You may want different ratios calculated than those specified in the original Ratio file record. There may have been an error in one or more of the entries that needs correcting. There are very lew conditions which require retransmission of data from the PC--I can't think of one right now, as a matter of fact.

Upon entering a 1, the program asks you to enter which record numbers in the Samp file you want to reprocess. You enter as many values as you like (150 is the maximum number; you are unlikely to need that many!), ending entry with a 0 . Zero is not a valid record number in Fortran and is often used to signal the end of input. The program will then calculate the samples in the order in which you entered their record numbers.

\subsubsection{Option 2 (reject runs)}

Enter 2 if you want to enter run numbers to be ignored in the calculations. This is a situation not often encountered; the most commonly rejected runs are those at the beginning or the end of the analysis. These are more conveniently eliminated by changing the first and last runs, respectively, in the Samp file than by entering their values explicitly using this option. If, however, there is a poor run in the body of the analysis that needs to be eliminated, this is the way to do it. An example of when this can occur is when there is an arc in the middle of an analysis. This often results in weird values for ratios and counts for the run in question. Upon entering the 2, the program will ask you to specify the run numbers you want rejected. It will continue to accept input until you enter a 0 for the run number. The run numbers entered will not be included in calculations of ratios and their averages in the program.

\subsubsection{Option 4 (individual channel list)}

This option provides the means of obtaining a list of the number of counts in each individual channel as stored in the Chdata file. When you choose this option, the program asks which run you want the list to start with.

It is unlikely you will need to use this option. It is included for trouble-shooting purposes. It is easier to inspect the individual channels using program File (Section 3.10.2) than it is to do it with $M S C$. Only if a hard copy is needed would it make sense to use this option.

\subsubsection{Options $3,5,6$, and 7 (combinations)}

To select more than one of the options described above, you must enter their sum as your choice for the $M S C$ option. Thus, if you want to recalculate and reject runs, you would enter $3(1+2=3)$. If you want all of them (unlikely), you would enter $7(1+2$ $+4)$. And so on. 


\subsubsection{Option 9 (list SAMP.DAT contents)}

This option allows you to list the contents of Samp on the terminal. You are not likely to want to do this, but on occasion it helps to locate a sample analyzed some time ago. It is of more use to the programmer and supervisor than to the analyst. You can enter starting and ending records in the Samp file so you don't have to get all 400 samples listed unless you want to. When the list is finished, the program asks you to enter a new option. If you don't want to calculate anything, just hit CTRL/C to exit the program.

\subsubsection{Option 10 (select output device)}

It is often convenient (especially when trouble-shooting) to direct output to the terminal rather than letting it go to the printer. Entering a 10 for your MSC option brings up a small window that asks for your selection of output device. These are summarized in Table 5.2.

Table 5.2 Output Device Numbers

\begin{tabular}{ll} 
Device & Description \\
\hline 6 & Printer in Back Lab \\
7 & Terminal \\
8 & TRU Lab
\end{tabular}

The reason for the apparently arbitrary choices is that 6,7 , and 8 are dictated by the output device identification numbers used in the Fortran programs. Any number other than 6,7 , or 8 defaults to 6 , the main printer.

After you enter your choice of output device, the program cycles back to the original menu to find out which samples you want to calculate.

\subsection{Interpreting output sheets}

Although it is the supervisor's responsibility to evaluate the results of an analysis, it seems appropriate to mention here some of the things he or she should look for. Nearly all evaluation of any analysis can be performed by inspection of the two output sheets generated by program $M S C$. These sheets are described below.

\subsubsection{First output sheet: Raw data}

The first output sheet lists the raw counts used in the calculations. Any results printed on the second sheet can be generated using these numbers and the corrections applied to them.

Each item listed is described below. The first two lines contain what might be called header information that helps in trouble-shooting should things not go as expected. 


\subsubsection{Sample Id}

This the ubiquitous six-character code identifying the sample. It is helpful here in case the two output sheets become separated; one can then match the raw data sheet with the results sheet.

\subsubsection{Input record no.}

This is the record number in the Samp file that holds data for the sample in question.

\subsubsection{CHDATA Rec}

This is the record number in the Chdata file where the raw channel-by-channel data start. There will be one record for every eight subgroups of data collected. For most samples, this will mean one record per run, but, for samples with more than eight subgroups, there will be two.

\subsubsection{SUMCTS Rec}

This is the record number in the Sumcts where the first run of condensed raw data starts. There is one record per run.

\subsubsection{Sweep}

The sweep rate in milliseconds. This is usually 2.0 , but there is no reason why other values can't be used.

\subsubsection{Cycles}

The number of cycles through the mass spectrum that constitutes one run.

\subsubsection{RATIO Rec}

The record number in the Ratio file for the information that controls which ratios are calculated. Its value cannot be 0 .

\subsubsection{SPIKE Rec}

The record number in the Spike file that holds spike information for an isotope dilution analysis. It will be 0 for other analyses.

\subsubsection{Corrected channels}

A warning is printed when an excessive number of channels in the raw data needed correcting. The statement looks like this:

NN channels were changed in run $X$, subgroup $Y$. Your results may be invalid. $\mathrm{NN}, \mathrm{X}$, and $\mathrm{Y}$ are numbers representing the indicated parameters. 
The seriousness of this message depends on the sample being run. Results for a Stable Isotopes sample, for example, where the demands for precision are not great and the elements often difficult of analysis, may still be quite acceptable even if the message appears for several runs. On the other hand, if you need the best possible analysis, you may need to rerun the sample.

The cause for this message is most often either poor peak shapes or misalignment of subgroup voltage windows with peak positions. In the former case, if refocusing doesn't correct the problem, the most likely explanation is a dirty source. Try a clean one. The latter case requires only that the subgroups in question be set to their proper positions. If most of subgroups are involved, adjusting the source high voltage is the best means to achieve this; if it is only one subgroup, its potentiometer setting needs to be shifted.

\subsubsection{Run}

The rest of the information on the first output sheet is in the form of a table. The left-most column of this table lists the run number associated with the data on the given line. These run numbers are those of the data as taken; that is, if you reject the first three runs of a twelve-run analysis, the numbers listed will run from 4 to 12 .

\subsubsection{Sum Chan}

This is the number of channels used to generate the sums of counts for each mass position for each run. The maximum number is 32 , which is the number of data points taken for each peak. Smaller numbers mean that at least one of the major peaks was slightly misaligned; this is seldom a serious matter as long as the number of channels is above, say, 25. Numbers too small indicate that something serious went wrong during the analysis.

\subsubsection{Raw Counts}

At the top of the next few columns are the mass numbers associated with the subgroups. They are listed in the same sequence as they were swept during data acquisition. These columns contain the number of counts calculated by taking the sum of the raw counts in the number of channels listed for the run in question; note that no correction has been applied for sweep factors. These sums have been corrected for some anomalous conditions, but no background, bias, or dead time corrections have been applied. It is these sums that are stored in the Sumcts file.

There is a lot of information available here should questions arise concerning the results listed on the second sheet. I will summarize only briefly to give a flavor of what should be looked for.

It is always a good idea to check the background position if you have any doubts at all about the results. If the background is unusually high, was this because of generally high values in every run or was it due to very high values in one or two runs? The latter condition is often indicative of noise spikes and should make you wary of ratios involving very lowabundance isotopes. Keep in mind that the number of counts in the background position is 
subtracted from all other positions. For isotopes of very low abundance, it can happen that there are more counts in the background than there are for the isotope.

Do the raw counts for each member of the pair of isotopes track with the run number? In other words, if, for example, the number of counts for the denominator is declining with time, does the number of counts for the numerator also decline? And, if it does decline, does it do so at about the same rate for each isotope? If you answer either of these questions no, check other isotopes. Can you discern a pattern? Do all isotopes but one behave about the same? If so, a contaminant is the likely explanation. If they do not, the possibility of an unstable ion signal should be investigated.

\subsubsection{Total Cts}

This is the number of raw counts collected for each isotope during the analysis. It is the sum of the numbers in the column associated with it. These numbers are helpful for making a quick evaluation of the data. For a more detailed analysis, the run-by-run counts are more valuable.

\subsubsection{Ratios of Avgs}

This line is provided for the convenience of the impatient analyst; it is really redundant. Printers can be very slow indeed when the results to be printed are crucial to an experiment. This line is the averages of all the ratios calculated; these will also be listed on the second sheet under the values of the ratios for individual runs.

\subsubsection{Second output sheet: Ratios and averages}

The second output sheet can actually be more than one piece of paper. This will occur when a lot of runs are taken or more than 11 ratios are calculated--there is room only for 11 ratios per line, and more ratios than 11 requires another output sheet. Since the information is the same no matter how many pieces of paper are used, I have chosen to define only the two output sheets.

The second output sheet holds header information, the values of ratios, their averages, and their statistics. It will also contain the results of isotope dilution calculations when appropriate. There follows a short description of the information on this sheet.

The first few lines hold general information; the body of the sheet lists the ratios for the individual runs; averages of ratios and their statistical attributes are listed near the bottom; isotope dilution information comes last.

\subsubsection{Descriptive title}

This variable is not specifically labeled due to space constraints. The maximum number of characters that can be held on one line is 132 , and identifying this variable with a label seems unnecessary. It is the first item listed after the general title ("ORNL MASS SPECTROMETRIC ANALYSIS"), appearing in the upper left corner of the sheet. 
This variable is intended to carry enough description to allow ready identification of what the sample was. NBS U-010 is an example of this type of information; for samples generated outside our group, the customer's sample identification should be included.

\subsubsection{Samp Id}

The unique six-character sample identification code.

\subsubsection{El}

The element symbol.

\subsubsection{Da}

The date of analysis. This is the date data were taken and not necessarily the date they were processed. It is read from the date in the PC at run time; it is not entered by the analyst.

\subsubsection{Rb Com}

This is the six-character code identifying the companion sample for a mixed resin bead analysis of plutonium and uranium. It is blank for other analyses. See Section 11.6 for more information.

\subsubsection{Sp Com}

This is the six-character code identifying the unspiked companion for an isotope dilution analysis. It is blank for other analyses. See Section 11.1 for a description of isotope dilution.

\subsubsection{IN}

The record number in the Samp file that holds information for the sample in question. IN stands for input.

\subsubsection{OUT}

The record number in the Out file to which results for this sample will be written.

\subsubsection{Batch}

The batch code. This two-character variable is used to identify which samples are to be subjected to special calculations or to help group together the samples from one project. It is blank if no entry was made. 


\subsubsection{Mch}

This is the identification number of the mass spectrometer on which the data were taken. These codes are defined in Section 3.9.4.1, Table 3.19.

\subsubsection{Tau}

Tau is first entry on the second line of output. It is the dead time; the Greek letter tau is traditionally used to represent it algebraically. It is in units of microseconds.

\subsubsection{BC}

$\mathrm{BC}$ stands for bias correction. It is listed as percent per mass.

\section{2.13 Cycles}

The number of cycles over the mass spectrum that constitute a run. It is defined in the Scan file on the PC.

\subsubsection{Swp Rate}

This is the sweep rate in milliseconds per channel. It is determined by the position of the switch on the Sweep Control Panel.

\subsubsection{Norm mass}

This is the normalizing mass used to indicate calculation of atom percents as desired.

\subsubsection{Temp}

This variable is actually the comments entered at the end of an analysis. The temperature should be included.

\subsubsection{Sums and Differences}

There can be up to three sums and three differences defined in the Ratio file. They are listed on the next lines, one sum and difference per line. Remember that the masses identified in Sum(1) are those used in calculating atom percents. Sum(2) alsu has special significance in some cases; it holds the isotopes of the unspiked sample in an isotope dilution analysis with no unspiked companion, for example. This matter is described more fully in Section 11.1.4.

\subsubsection{Subgroup}

The subgroup numbers (1-N for $\mathrm{N}$ subgroups) appear on the next line. Below them is information associated with the various subgroups. If $N$ exceeds 8 , there will be two sets of lines holding this information; data for 8 subgroups is all that one line can accommodate. 


\subsubsection{Mass}

The mass numbers appear on a single line.

\subsubsection{Sweep Factor}

The sweep factor for each subgroup is listed.

\subsubsection{Bias correction}

The bias correction applied to the counts for each mass position is listed. One value will always be 1.000 ; this is called the base mass for bias correction and its position is defined in the Ratio file. Other bias corrections will be greater or less than 1.0 depending on whether the mass of the subgroup is greater or less than the base mass. Note that the bias correction factor for the background position is always 1.0. Other correction factors are calculated from the bias correction per mass and the mass difference between the isotope in question and the base mass. Here is the equation:

$$
\text { bias }=1.0-b c *(m b-m i)
$$

where bias is the correction factor to be applied to that mass position, bc is the bias correction per mass, $\mathrm{mb}$ is the base mass and mi the mass of the ith isotope. The counts for each mass position are multiplied by the associated bias.

\subsubsection{Corr. Counts}

These are the total number of counts collected during the analysis for each mass position. Except for the background posicion itself, they have had all corrections applied to them. This includes, of course, the sweep factor; note that the raw counts listed on the first sheet are not corrected for sweep factors. Dead time and bias corrections are applied to all positions without exception. Every position besides the background has had the background subtracted as well. Subtracting the background from the background position would obviously give 0.0--not very informative. Instead, the number of counts for the background position is the number of counts collected for it during the analysis.

A second line of corrected counts will appear if corrections are made for contaminant elements.

\subsubsection{Run}

The next portion of the second output sheet lists ratios calculated for the individual runs. The run number in the left-most column will always start with 1 (unlike those on the first output sheet) and go up through however many runs were included in the calculations. 


\subsubsection{SCh}

This is a terse abbreviation of sum of channels; listed below it are the number of channels used for each run to calculate the sums of counts for each mass position. These are identical to those listed on the first output sheet.

\subsubsection{Total Cts}

This sort of total counts is to be distinguished from those appearing as corrected counts at the top of the sheet. These values are the number of counts collected in the designated run; those above are the total counts for the analysis for each mass position. These sums integrate counts over all isotopes for one run; the other integrates counts over all runs for one isotope.

\subsubsection{Ratios}

Each ratio appears under a heading that tells which ratio was calculated. Ratios larger than 99.99999 are too big for the output field and appear as a string of asterisks $\left({ }^{* * * * * * * *}\right)$; this is a Fortran convention and not the programmer's choice. (Note that Asterix, like Obelix and Dogmatix, is a cartoon and not an ASCII character. ${ }^{*}$ is an asterisk.) Values for ratios too large for the field appear in $\mathrm{E}$ (exponential) fields immediately below the individual ratio section. Ratios whose values have been statistically rejected are indicated by a single asterisk after them. There is room for 11 ratios on a line; if more than 11 ratios are called for, another output sheet is used. The maximum number of ratios is 22 . Remember that $999 / 999$ is the secret code for the isotope dilution ratio; see Section 11.1.

\subsubsection{Avg}

This line holds the averages of the individual run ratios. All values are included except those marked with an asterisk.

\subsubsection{Statistics}

The next three lines hold the standard deviation, the standard error of the mean, and the $95 \%$ confidence interval for each ratio. Note that the standard error of the mean is the standard deviation divided by the square root of the number of runs; it will always be smaller (i. e., better) than the standard deviation. It is for this reason that commercial vendors tend to report standard errors of the mean. This and other statistical issues are addressed in Section 11.7.

\subsubsection{At Pct}

The next four lines hold the atom percents with their associated statistics. Atom percents are calculated along with other ratios, as described in Sections 3.5.2.5 and 3.5.2.7. The isotopes identified in sum(1) are used as numerators; the sum of the counts of these isotopes is the denominator. All statistical parameters calculated for atom percents are calculated on the same basis as are those for the ratios listed in the previous section of the output sheet. 


\subsubsection{Wgt Pct}

The isotopic composition in weight percent appears on a line below atom percents. It is calculated from the average atom percents, and its statistics are identical to those for atom percents. Some projects require results in weight percents. The atomic weight of the sample appears at the end of this line.

\subsubsection{Corrected sample isotopic}

For those cases where a corrected sample isotopic composition is calculated, there are four lines containing relevant output. The first serves as a header and holds the mass positions. It is followed by lines listing corrected counts, the corrected sample isotopic composition (in atom percent), and the percent relative standard deviation.

Examples of when this output will appear are for an isotope dilution analysis for which no unspiked companion is available and for an analysis for which there is a contaminant element correction. See Section 11.1.4.

\subsubsection{Isotope dilution output}

The bottom portion of the sheet (or the top of a new page if one is needed) contains isotope dilution calculation information.

\subsection{Spike Isotopic}

The isotopic composition of the spike is listed, along with its atomic weight.

\subsection{Other spike parameters}

There are two lines that hold the rest of the relevant spike information. Each number has a label, so there should be no confusion about what is what. They appear in the following order: Spike, sample, dilution, and aliquot weights; density; dilution factor; concentration in units of weight; concentration in terms of volume; total aliquot weight of the element; and the standard deviation for this last value.

The two concentrations are labeled $\mathrm{Xg} / \mathrm{g}$ and $\mathrm{Xg} /$. This is to indicate that the units are defined by the those of the sample and spike weights. The units of the spike determine the units of the numerator of the concentration; the units of the sample determine the units of the denominator. If you enter your spike weight in nanograms and your sample weight in grams, the concentration will be in nanograms per gram (ppb).

The concentration on a volume basis will be equal to that calculated on a weight unless a value is entered for the density. Volume-based concentration calculations are inherently less reliable than those based on weight because density is difficult to measure and varies as a function of temperature. 
See Section 11.1 for a more detailed description of isotope dilution calculations.

\subsubsection{Interpretation}

There is a wealth of information on this second output sheet; only some general guidelines concerning interpretation will be given here.

The first thing to do is to look at either the averages of the ratios or the atom percents. Do they make sense? If you are expecting natural uranium and the report says its $25 \% 235-\mathrm{U}$, there is a problem somewhere. Let me point out that it may not be in our lab. Customers have been known to be wrong, although they resist acknowledging it.

The next thing to look at (assuming all is well so far) is the precision of the measurements. Are they about what you would expect? If not, you should look further to try to isolate the problem. I'll remind you that inspecting ratios for problems is almost always more fruitful than looking at atom percents. A poor value for one atom percent affects all the others; using ratios will often allow you to isolate the problem to one mass position. For example, if the standard deviation for one ratio is suspiciously high, are all the others high, too? If they are, you probably have a generally poor analysis. If they aren't, then you probably have a problem with just the one isotope; this is often a contaminant. Look at all suspicious ratios (and even all ratios, period) for trends. A monotonically increasing or decreasing ratio is bad news. It means either excessive isotopic fractionation occurred during the course of the analysis or a contaminant was present.

Check the total counts for each run. Step increases indicate refocusing and, often, an increase in temperature. Ratios sometimes show different values after this activity. An analysis with several such steps and sharp declines in signal intensity between them is usually not a very good one.

If an isotope dilution analysis is under consideration, check the concentration. Does it make sense? If you were expecting a concentration in nanograms per gram and got a result in micrograms or milligrams, look for erroneous weight input.

These are only a few of the things to look for; it would be impossible to codify them all, even granted I could remember them off the top of my head. Remember that a decision on reanalyzing a sample is in part determined by how good an analysis is required. If precisions of $1 \%$ are all that's needed, reanalyzing should not often be necessary. On the other hand, if precisions of a few tenths of a percent are required, or if low-ppm abundant isotopes are to be measured, then we need to be sure we have done just as well as we possibly can. 


\title{
6. PROGRAM REP
}

Program Rep is designed to generate report sheets that consolidate results for groups of samples into a single table. It will also calculate averages of replicate analyses and store them for use as companion samples for isotope dilution calculations.

It is important to remember that the Out file is the only file accessed by Rep. Manipulation of other files is accomplished by invoking program File (Chapter 3).

A menu window is opened upon invoking Rep. It looks like this:

\section{Table 6.1 Rep Menu}

\author{
Report Menu \\ 0 to EXIT program \\ 1 to edit Out.dat \\ 2 to average specified samples \\ 3 to list specified samples \\ 4 to list permanent companions \\ 5 to list analyses of standards \\ 6 to list by batch code \\ 7 to list by element \\ 8 to list sample id codes only \\ 9 to list all samples between limits \\ 10 to correct $\mathrm{Pu}$ to a given date
}

The result of selecting each of these options is described in the next sections.

\subsection{Option 0 (EXIT)}

As with other programs, choosing 0 from a menu of this type signifies that you are done with the program. You are returned to the monitor, which will display the prompt $(\$)$.

\subsection{Option 1 (edit)}

The editing routine called from Rep is identical to that called from File. See Section 3.11 for a description of how to edit the Out file.

\subsection{Option 2 (average specified samples)}

It is often desirable to obtain the average of replicate analyses. Rep will do this for you if you tell it which samples you want included in the average. You are asked whether you want to identify samples included by their record numbers in the Out file or by their sixcharacter identification codes. A maximum of 300 samples can be included in an average, 
safely beyond any reasonable requirement. Entry of sample identification is terminated by a 0 or blank. You must use blanks (just hit Return) if you are entering sample identification codes; either a blank or a 0 will work if you are entering record numbers. Averages of all ratios and atom percents, together with their standard deviations, are calculated and listed on the printer. The values averaged are also listed.

The program does little checking. It assumes you have used the same Ratio file record number for all samples. If this is not the case, confusion (or possibly even chaos) will result.

One of the uses of this option is to make entries in the Out file of the information necessary for companion samples. Once an average is calculated, you are asked for a sixcharacter sample identification code. Hitting Return (or Enter) has the effect of filling this variable with blanks; if you do this, the averages will not be stored. If you enter anything else, the averages will be stored in the Out file under the code name you entered. One convenient use of this feature is to use it to store the averages of replicate analyses performed to determine the isotopic composition of the unspiked companion sample for isotope dilution calculations.

\subsection{Option 3 (list specified records)}

Selecting this option will result in a list on the printer of the results of the samples you specify. You may enter either record numbers or sample identification codes (but not both for a single list--have a heart!).

\subsection{Option 4 (list permanent companions)}

Selecting this option will produce a list of sample identification codes in the first 50 records of the Out file--the so-called "permanent companions." This can also be done from editing Out using either program File or program Rep.

6.6 Option 5 (special standard list)

Choosing this option will result in a list of the results of all standards that are identified by having a batch code of ST. This may be helpful for QA purposes. You can specify the limits in Out file record numbers between which you want standards listed.

\subsection{Option 6 (list by batch code)}

Choosing this option will produce a list of all samples analyzed under the same batch code. This is very handy when a number of samples (such as Portsmouth pine needles) are submitted for the same project.

\subsection{Option 7 (list by element)}

This option produces a list based on element symbol; you are also allowed to choose the limits (in terms of record numbers in the Out file) of the search; this eliminates unwanted output. 
6.9 Option 8 (list sample id codes only)

This option produces a list of the six-character sample identification codes correlated with their record numbers. You may choose initial and final record numbers so you don't have to wait for all 3000 samples to be listed.

6.10 Option 9 (list all between limits)

This is primarily a trouble-shooting aid. It will produce a list of all samples between the Out records you specify.

6.11 Option 10 (correct Pu to specified date)

This allows you to correct Pu results to a given date. You can also accomplish this by entering the date in the Samp file (Section 3.9.2.10) and recalculating. Details of this operation are given in Section 8.3. 


\section{PROGRAMS TRAN AND REC}

Tran (for TRANsfer) resides on the PC's. It works in conjunction with program Rec (for RECeive) on the VAX. Together they effect the transfer of data from the PC's to the VAX. Tran is actually a batch file. To identify which data to transfer, Tran searches the Samp file for those records that have a value for icalc (a.k.a. calculation index) of 0. It will transfer the information in Samp and the raw channel-by-channel data in Chdata to the VAX. The correct record in Chdata with which to start is one of the pieces of information in Samp; Tran starts with that record and proceeds in sequence through Chdat $a$ until it has transferred the amount of data specified by the number of runs and the number of subgroups. Data for eight subgroups can be stored in one record of Chdata; more than eight subgroups require two records.

To initiate transfer, enter Tran on the PC's terminal. In most cases this will be all that is necessary; Rec should be resident on the VAX. Rec will receive the data, store it in temporary files, and transfer it to permanent storage in the Samp and Chdata files in the MSCALC directory. Once this is accomplished, program MSC is automatically executed; results for the samples whose data were just transferred will appear on the main printer or on the printer in the TRU laboratory, wherever the mass spectrometer in question is located.

All of this is supposed to be invisible to the analyst. All bookkeeping chores on both computers are done by the software.

There is an occasional hang-up. This is often due to problems reported on the printer devoted to the transfer process. If you fail to get output in a reasonable time, check this printer. If an error condition has been reported, hit CTRL/C (Control and C simultaneously) to get out of the program currently in memory. You should be returned to the monitor as indicated by the prompt (\$). Enter @RECMSC. If the error was your only problem, the program will now proceed automatically with its business; it should now produce the output sheets and cock itself to receive another sample.

The (a) character is DEC's way of accessing a batch file. RECMSC calls for sequential execution of the receiving program, $R e c$, and the data processing program, $M S C$. The VAX lies more or less dormant in $R e c$, checking periodically to see if anyone wants to transfer data. When the flag to transmit is raised, it accepts data, stores it on the VAX, invokes $M S C$, and returns to its wait state. More than one PC can be transmitting simultaneously, although of course the VAX receives the data one sample at a time. @REMSC should be entered every time the computer has to be booted.

It is occasionally necessary to reinitialize the receiving files. This is accomplished by executing a program called Init_Status.

Problems that can't be corrected as indicated above will unfortunately have to be referred to a computer expert. 


\section{MISCELLANEOUS PROGRAMS}

There are three programs that can be useful that are not part of what might be considered the main stream of calculations. They are not designed specifically for treating data from the ORNL-designed mass spectrometers, and can be used on data from any source. The programs are summarized in Table 8.1 and described in the sections that follow.

\section{Table 8.1 Miscellaneous Programs}

Program

Atwtpc

Ida

Putz
Description

Converts atom to weight percent.

Performs isotope dilution calculations

Converts Pu compositions from one date to another.

\subsection{Program Atwtpc}

This program converts atom percents to weight percents. It uses the exact mass information in the Massab tile, so there must be an entry there under your element symbol. Upon invoking the program, you are given your choice of output device (6 for printer, 7 for terminal). A window then opens to ask for input. An example of it in its early stages is shown in Table 8.2.

\section{Table 8.2 Initial Input Window for ATWTPC}

1. Sample id code

2. Element symbol, blank to stop

3. Input index

4. Mass for denominator
Test case

$\mathrm{U}$

2

Use 1 to enter ratios, 2 for atom pcts.

\subsubsection{Sample id code}

This is a descriptive title and can be anything you like.

\subsubsection{Element symbol, blank to stop}

This is the two-character symbol that must match an entry in the Massab file. The program cycles until you enter a blank for this variable. 


\subsubsection{Input index}

This is an index used to indicate how you want to enter your information. A 1 will tell the program to accept input as ratios; a 2 (as illustrated above) will tell it you are entering atom percents.

\subsubsection{Mass for denominator}

An entry is required here only if you plan to enter ratios, as signified by entering 1 for the input index. If you enter a 2 for the input index, the request for input of this variable will be skipped. The value entered here is then the mass for the denominator of the ratios--all ratios must have the same mass for the denominator for the calculations to proceed. A typical case would be to use 238 for uranium.

Upon reaching this point, the program reads the appropriate entry in the Massab file and displays the appropriate prompts for input of ratios or atom percents. An example of the input window at this stage is shown in Table 8.3.

\section{Table 8.3 Final Input Window for ATWTPC}

1. Sample id code

2. Element symbol, blank to stop

3. Input index

4. Mass for denominator

Enter atom pcts

5. 233

6. 234

7. 235

8. 236

9. 238
Test case<smiles>[AlH2]</smiles>

2

Note that mass numbers have been added to the window shown in Table 8.2. For atom percents, you will be prompted for an entry for each isotope. Ratios require one fewer entry since the ratio of the mass in the denominator to itself is by definition 1.0.

You are given the opportunity to correct any erroneous entries. As with all the programs, this is achieved through entering the index number that corresponds to the variable you want to change. To alter the atom percent of 2.35 in the example above, you would enter 7. The cursor will move to the appropriate place in the window and ask for a new value. Once this is entered, you will be asked for another index value. This will continue until you enter 0 for your index.

When all data are correctly entered, the calculations are performed and the results listed on the device you chose earlier. Ratios and atom and weight percents are listed. 


\subsection{Program Ida}

Ida stands for Isotope Dilution Analysis. This program is not normally needed for data taken using ORNL instruments because the necessary calculations are an integral part of the programs (File, MSR, MSC). Such calculations are not available on either the IMMA or the VG-354, and this program is designed to eliminate the need to go through the tedium of performing them manually.

The menu for Ida is illustrated in Table 8.4.

\section{Table 8.4 IDA Menu}

Select option

0 to exit

1 for input from disk files

2 for manual input

3 to list instructions

Choosing option 3 results in a rather terse set of instructions being listed on the terminal. These are listed in Table 8.5. Option 0 terminates Ida and returns control to the monitor. The other two options are the ones to use to perform the calculations.

\section{Table 8.5 IDA Instructions}

\section{IDA--Isotope Dilution Analysis}

Input may be either from files already existing on the disk (SPIKE and OUT) or by strictly manual entry. If the former is used, the record numbers in those files are required and must of course contain the necessary information for the spike and the unspiked sample.

If you wish to perform IDA with no unspiked companion, enter a 0 (zero) for the record number for the Out file.

Criticisms (friendly only) and comments should be directed to
D. H. SMITH 


\subsubsection{Option 1 (input from disk files)}

This option gives you the convenience of using data already in the Spike and Out files. This is especially convenient if there are a number of calculations to be made; it is relatively simple to enter companion information into the Out file (Section 3.11) and spike information into the Spike file (Section 3.7). The only information required for the calculations are the various weights and the values of the isotope dilution ratio in the mixture.

\subsubsection{Option 2 (manual input)}

If spike and unspiked sample information isn't in disk files, it must be entered manually. An example of an input window is shown in Table 8.6.

\section{Table 8.6 Input Window for IDA}

Manual Input

1. Mass for numerator

2. Mass for denominator

3. ID ratio for spike

4. ID ratio for unspiked sample

5. Atomic weight for spike

6. Atomic weight for unspiked sample

7. Atom pct denom, spike

8. Atom pct denom, unspiked sample

The meanings of the prompts should be made clear by referring to the section describing isotope dilution calculations (Section 11.1). ID stands for isotope dilution. ID ratio means the measured ratio and not the masses of the isotopes used to calculate it.

As usual, you are given the opportunity of correcting erroneous entries through selection of the appropriate correction index (1-12) in the example above. The program moves on only after 0 is entered for this index. A new window will open, and you will be asked for the isotope dilution ratio of the mixture. You may enter up to 10 values, which would represent as many replicate analyses.

The output sheet lists all input plus the calculated concentrations. If more than one value for the mixture ratio was entered, the average and standard deviation will be listed as well. 


\subsection{Program Putz}

The function of this program is to calculate the isotopic composition of plutonium at a given date. 241-Pu has a half-life of about 14.5 years and will decay enough to have a significant effect in only a few months. For example, NIST Pu-247 has decayed from $4.54 \%$ 241-Pu when it was certified in 1971 to about $1.7 \%$ in August 1992. This feature is available in several other programs, including MSC. It is most often used when results for an interlaboratory experiment must be reported as of a given date to allow valid comparisons.

Input is straightforward. You are given the opportunity to correct NIST Pu-947 to today's date; if you want anything else, you must enter the two dates (the date for which you know the composition and the date for which you want it) and the known isotopic composition. The output sheet lists both compositions in atom and weight percents; the atomic weights are listed as well. equation:

Radioactive decay follows a first-order rate law that can be expressed by the following

$$
C_{1}=C_{0} \text { e }-\left(0.693 t / t^{1 / 2}\right)
$$

where

$$
\begin{aligned}
& C_{1}=\text { concentration at time } t \\
& C_{0}=\text { initial concentration at } t_{0} \\
& t=\text { elapsed time } \\
& t_{12}=\text { half-life of the isotope }
\end{aligned}
$$

This equation is implemented in a subroutine that should be invisible to the analyst. 


\section{COMPUTER CONSIDERATIONS}

Since we would be out of business without computers, I thought it advisable to include a chapter devoted to them. Three instruments are linked via DEC's Ethernet to a central MicroVAX computer. Each of the instruments has a DEC 316 (IBM PC clone) dedicated to its operation. The PC's control acquisition of data and serve as communications links to the MicroVAX. Once data are collected, they are transmitted to the MicroVAX, where all calculations are performed. It is thus necessary to have at least two computers operational for an analysis: the PC on the mass spectrometer you want to use and the MicroVAX.

The MicroVAX is a far more powerful and complex instrument than we have ever had before. Most systems of this complexity have a person, called the system manager, whose job it is to manage the computer. We cannot afford this luxury, but we don't really need it. Our needs are child's play for the VAX, and we can get by knowing only a very small subset of the system commands.

\subsection{Description of the DEC MicroVAX}

Our MicroVAX has $13 \mathrm{Mb}$ of RAM and two hard disks: one of these latter has $42 \mathrm{Mb}$ of storage, the other $159 \mathrm{Mb}$. There is no reason to anticipate that we will ever become system-limited, as we were with the PDP-11/23. One of the plans to be implemented after our move to X-10 is to integrate the VG-354 to the VAX.

Programs and files for processing mass spectrometric data reside on the larger disk. Programs are written on the assumption that the system will be booted on this drive.

\subsection{Booting the MicroVAX}

The MicroVAX requires rebooting every time the power fails. While this is the most common cause of rebooting, there will be other times when it becomes necessary.

To determine the disc on which the system is booted get a directory at the prompt. The command line will look like this:

\section{$\$ \operatorname{dir}$}

where the $\$$ is the DEC prompt and you enter the "dir." A directory will be listed on the terminal, and the first information out will tell you which disk is being used. DUA0: is the smaller disk; you want DUA1: the larger one. The steps to get booted properly are summarized in Table 9.1. 


\section{Table 9.1 Rebooting the MicroVAX}

1. Press HALT on DUA0:. The green light comes on.

2. Press HALT again. The green light goes out.

3. The switch on the back of DUA0 should be up (left).

4. Press RESTART on the front of DUA0:. The screen will show a decrementing number (e.g., 7..6..5..). Be patient. This takes several minutes.

5. The screen will show $>>>$.

6. Enter BO DUA1:. Hit Enter or Return.

7. The screen will display 3..2..1

8. Change the switch on the back of DUA0: to down (right). This allows the terminal to be turned off without disrupting the system.

Note that this is not the world's fastest boot. It takes about five minutes.

\subsection{Directories}

As on most computers, DEC's disk drives are partitioned into directories. As disk storage increases seemingly without bound, the task of organizing the information so you can find it becomes more and more challenging. Creation of directories and subdirectories and sub-subdirectories and etc. is an attempt to bring order to chaos. The only directory with which you need concern yourself is called MSCALC (for mass spec calculate). All programs and files required to process mass spectral data reside in this directory. If you find yourself in the wrong directory, it will be necessary to move to the correct one. To identify your directory, you can either type dir at the prompt (\$) or you can type SHO DEF (for show default). The former command will generate a list of the files in the present directory in addition to its name; the latter will list only the directory name on the terminal. If you are not in MSCALC, you should change directories by entering SET DEF [MSCALC]. The square brackets are essential; the period is there only for the grammatical demands of the sentence--all sentences end in one, or so I was taught; the period must be omitted on the terminal.

Only executable fiics and data files are here. The former end with the .EXE extension, the latter with .DAT. Ex?mples are MSC.EXE and SPIKE.DAT. Editing a Fortran program requires a file with the .FOR extension; a compiled module has the OBJ extension. These files are located in other directories (DHS, MCK) and are not accessible from MSCALC. Editing should only be attempted by someone who knows what he or she is doing.

\subsection{Input fields}

Some input requires that it be made in specific fields. This section will describe Fortran specifications as an aid in those instances. 
A Fortran field specification has the general form of NLn where $N$ is the number of fields, $\mathrm{L}$ is a letter code designating what sort of input is expected, and $\mathrm{n}$ is the width of the field in characters. Sometimes the $\mathrm{n}$ part is specified as $\mathrm{n} . \mathrm{m}$, where $\mathrm{n}$ is the field width and $m$ the number of places after the decimal point.

Let's consider data types. The three we'll worry about are integer, real, and character.

\subsubsection{Integers}

Integers are numbers like the ones you use to count the number of hours left before you go home. $1,2,3,4 \ldots$ are integers. So are $-333,23795$, and so on. An integer field is designated by an I in Fortran. 13, for example, says the computer will accept an integer of up to three digits as input for that field. Note that the width specification must include the sign, so I3 will accept integers from -99 to 999 . Commas, decimal points, and any characters other than 0-9 are invalid and won't be accepted. There must be a character for each of the three positions in the field; remember that space is a character.

If the input prompt tells you 213 fields are to be used, you would enter (for example) 235238 to get that isotopic ratio. Other valid entries (at least from the computer's point of view; it's up to you to be sure they make sense) are 063065 or 6365 (the leading zero can be either an explicit 0 or a blank); 123456; 11165 .

\subsubsection{Real numbers}

Real numbers are distinguished from integers by having decimal points. They are no more real, of course, than integers, where decimal points are not allowed. The distinction between the two is made because computers can perform integer arithmetic far more efficiently than they can real. So can you. It's a lot easier to perform $2 \times 3$ than it is $2.456787 \times 3.765432$.

Real fields have the form NFn.m or NEn.m. The $E$ field is used for numbers conveniently represented as exponents of 10: 6.023 E23 is a familiar example. There is no distinction in the computer between the two forms: all real numbers are stored in exponential format no matter how they are entered. Thus, for example, a number entered as 9.33 is kept in the computer memory as $0.933 \mathrm{E}-1$. You may even enter the E explicitly on input for both $\mathrm{F}$ and $\mathrm{E}$ fields. Let's look at a couple of examples.

Suppose the input field is F10.5. This means that the field is 10 characters wide and that, unless you enter a decimal point yourself, there will be 5 places after the decimal point. A decimal point you enter always supersedes the field specification. So, if you enter 1234567890 , the computer thinks you mean 12345.67890 . If you enter 123.4567890 , that will be what the computer will have. If you enter $1.23456 \mathrm{E} 2$, the computer will hold that value (123.456).

All you should have to do if prompted for a real number is to enter it in the most natural way. Only very large or very small numbers will be easier if you use the $E$ character. 


\subsubsection{Character fields}

Character variables are often called strings in other languages; such variables hold any sequence of ASCII characters. This is the variable type of the six-character sample code, the 20-character descriptive title, the two-character element symbol, and numerous other variables for which it would make no sense to use numbers, real or integer.

The only thing to worry about with character variables (aside from accurate typing!) is the width of the field. This is defined by the box that appears adjacent to the prompt; as long as you stay within it, you will be all right. The software is written so you cannot go beyond the box; any characters you enter after you reach that limit are ignored. 


\section{OPERATING A MASS SPECTROMETER}

This manual is not intended to provide instructions for operating a mass spectrometer. That matter was covered in a previous publication. ${ }^{9}$ It seems, however, that it would not be out of place to summarize the interaction of an instrument with the new data systems.

\subsection{Getting started}

Your first move when you have samples to analyze is to get everything turned on. This includes booting the PC on the instrument you are going to use. The Sweep Control Panel must be active to execute $M S R$ or an error message appears on the terminal to prompt you to turn it on. You cannot proceed until you pass this test. To take data, of course, there are many more switches that have to be activated: the NIM bin, the source high voltage supply, the sweep amplifier, etc. And remember to open the slit valve!

You must know which Scan and Ratio file record numbers you are going to use before you can complete entering all the required information for MSR; see Table 4.1 for an example of an input window. If you are going to perform an isotope dilution analys: you must know the Spike file record number and all the weight information associated with the spike and sample. There are other analyses that require prior knowledge of various parameters. It is the analyst's responsibility to have this information.

\subsection{Using MSR}

To initiate data acquisition, enter $M S R$ from the keyboard. A window will open filled with prompts for various parameters--see Table 4.1. Enter all relevant information as described in Chapter 4. Once all data have been correctly entered, control shifts to the interactive mode where the PC and mass spectrometer are communicating. You must have the Sweep Mode switch on the Sweep Control Panel set to Wait before it will let you proceed beyond the mere display of the message you encounter if you have it set anywhere else. The terminal will display some of the information you entered and some from the Scan file record number you chose. If anything is incorrect, get out of the program and start again. You can leave the program by hitting CTRL/C on the keyboard or by setting the Sweep Mode switch to Store and hitting the End Sample button.

Bring in the ion beam and focus it; when all is well, start taking data. New information will be displayed at the conclusion of each run; this includes the count rate of the most intense peak and the values of up to three ratios. This matter is covered in more depth in Section 4.2.

\subsubsection{The Sweep Control Panel}

The Sweep Control Panel is the heart of the data-taking system. It serves as the interface between the analyst and the mass spectrometer, using information from the Scan file as its guide. 
The Sweep Control Panel displays various parameters during the course of the analysis, some of which are written into the Samp file. Among the items displayed are the run number, the cycle number, and the subgroup number. These values, described below, can be used to monitor the progress of an analysis.

\subsubsection{Subgroup number and the 8-16 subgroup switch}

The left-most portion (two digits) of the large red LED display indicates which subgroup is presently being monitored. Subgroup numbers run from 11 to 18 and from 21 to 28. The first digit represents which bank of subgroups is being used. Subgroups 1 through 8 comprise the first bank; 9 through 16 are the second. Access to the two banks of potentiometers is controlled by the small unlabeled toggle switch between the sweep count LED display and the Single SG Select switch. In the left position, access to the first bank (subgroups 1-8) is given; in the right, access to the second bank (subgroups 9-16) is given.

Convention has it that uranium is controlled by the first bank by default; all other elements are controlled by the second. This is an arbitrary choice made for the analyst's convenience; it can be changed or eliminated if desired. The idea behind it is that, since uranium has been historically far and away our most common analyte element, it would be convenient to reserve one bank of subgroups for it alone. This preserves the potentiometer settings from one uranium analysis to the next and reduces the hassle involved in setting up. This system breaks down, of course, when more than eight subgroups must be used; then all 16 subgroups are accessible. Use the toggle switch to select the appropriate bank.

\subsubsection{Cycle number}

The three central LED's display the current cycle number (labeled Sweep Count). Each run is defined in the $S c a n$ file to have a given number of cycles. This value is displayed on the terminal screen. Comparing the value displayed by the LED's to that of the total number of cycles shown on the terminal tells you how far along in the present run you are. The number displayed by the LED's will increment until it reaches the preset value; at that point the run is over. The data accumulated in the computer memory are stored on the disk, the cycle counter is reset to one, and data accumulation for the next run starts. Note that, at the end of each run, both the Samp and Chdata files are updated. This provides insurance against the loss of data. For example, if an arc locks up the computer and requires a reboot, the only data lost are those of the run being accumulated at the time of the arc; data for all previous runs are safe on the disk. The only way to iose those data would be to lose the hard drive on the PC.

\subsubsection{Run number}

The two right-most LED's display the run number. Ten runs normally comprise an analysis, but this number was chosen because we have ten fingers--there is nothing sacred about it. Historical wisdom dictates that 15 or 20 minutes of data-taking are usually required to get a good analysis; the number of cycles is chosen to provide this length of time for ten runs. The maximum number of runs that can be taken for one data set is 19; the program will automatically cease data-taking after 19 runs. If you want fewer than 19 , as you normally will, push the "End Sample" switch on the upper right corner of the Sweep Control Panel. 
The computer eliminates the run it is currently taking and calls for end of sample information, as described in Section 4.2.12.

\subsubsection{At the end of a sample}

Once the information concerning first and last runs is entered, you will be given a choice of your next step. You may exit the program, run another sample with the same parameters, or run an entirely different sample. If you choose to run a sample with the same parameters (helpful if you are running replicate filaments), you have to enter only the bare minimum of information: sample identification code and title. All other parameters, including spike information if there is any, remain the same. If you want to take more data from the same filament, just enter a new sample identification code and resume running.

\subsubsection{Sweep rate switch}

The value of the setting for the sweep rate switch is stored by the computer in the Samp file. Data-taking has traditionally been carried out at $2 \mathrm{msec}$ per channel, but this is not a necessity. The fastest sweep rate $(0.2 \mathrm{msec} / \mathrm{chan})$ is not recommended because the switching time between channels becomes a large enough fraction of the total time to become a problem. Bear in mind, however, that, to achieve the desired analysis of time of 15-20 minutes, other parameters will have to be adjusted to compensate for the change in sweep rate. Either the number of cycles per run or the number of runs will have to be altered. The maximum number of runs is 19 , so you are limited in what you can do with this parameter. There is, however, no compelling reason to use a sweep rate other than the conventional 2 msec/chan.

The sweep rate is a constant for a given analysis; there has never been a need for it to be a variable, although there is no reason in principle why it couldn't be. This means that, should you inadvertently take the first run at a sweep rate other than $2 \mathrm{msec} / \mathrm{chan}$ and want to correct it on subsequent runs, it will be necessary for you to hit the "End Sample" switch and start a new sample. This is somewhat awkward but no big deal. 


\section{SPECIAL TOPICS}

This chapter covers a potpourri of subjects that I felt were important enough to warrant separate attention.

\subsection{Isotope Dilution}

Isotope dilution is a very powerful technique for determining the amount of an element present in the sample. It is the most accurate quantitative method available for many cases, particularly at low concentrations. It is a very important aspect of our work, and consequently it is important for everyone to understand how it works, at least in principle. For an isotope dilution analysis, a known amount of an isotopically enriched spike is added to a known amount of sample, whose isotopic composition is different from the spike's; the isotopic compositions of both spike and sample must be known. The ratio of the sample isotope to the spike isotope in the mixture of spike and sample is measured. This value, along with various other parameters, is fed into the isotope dilution equation and the concentration of the element in the sample calculated. The details of this calculation are discussed below.

\subsubsection{The isotope dilution equation}

The isotope dilution equation has several algebraically equivalent forms; we use one first developed by Hintenberger. ${ }^{10}$ Here it is!

where:

$$
C=\left(W_{1} / W_{s}\right)\left(A_{s} / A_{t}\right)\left(a_{t k} / a_{s k}\right)\left(R_{m}-R_{t}\right) /\left(R_{s}-R_{m}\right)
$$

$$
\begin{aligned}
& C=\text { concentration } \\
& W=\text { weights } \\
& A=\text { atomic weights } \\
& a=\text { atom percents (or atom fractions) } \\
& R=\text { ratios }
\end{aligned}
$$

and the subscripts are defined as follows:

$$
\begin{aligned}
& \mathrm{s}=\text { unspiked sample } \\
& \mathrm{t}=\text { spike (tracer) } \\
& \mathrm{m}=\text { mixture of sample and spike. }
\end{aligned}
$$

The ratios $\left(R_{x}\right)$ are defined:

$$
\mathrm{R}_{\mathrm{x}}=\mathrm{a}_{\mathrm{xi}} / \mathrm{a}_{\mathrm{xk}}
$$

where $\mathrm{x}$ is $\mathrm{s}, \mathrm{t}$, or $\mathrm{m}$ as defined above and $\mathrm{i}$ and $\mathrm{k}$ refer to the two isotopes of the isotope dilution ratio. Isotope $i$ is usually the isotope of high abundance in the sample and $k$ the isotope of high abundance in the spike, but using them (consistently!) in the reverse definition is valid algebraically. For example, for uranium, $i$ is usually 238 and $k 233$. 
There are several things to note about this equation and how it applies to what you want for your analysis. One is that the right side has no units except for those derived from the weight terms $\left(W_{1} / W_{s}\right)$. The units you use for these two weights define the units of your concentration. For example, if you enter your spike weight in micrograms and the sample weight in grams, your concentration units will be micrograms per gram or ppm.

Note that all the terms except those containing $R_{m}$ are constant for a series of replicate analyses. All the terms preceding the $\left(R_{m}-R_{t}\right) /\left(R_{s}-R_{m}\right)$ expression can be lumped together in a single constant in this case. This is only helpful if you have to perform the calculations by hand, but, believe me, this happens.

The ratios $R_{x}$ are called the isotope dilution ratios. They are the ratios of the abundance of sample isotope to that of the spike isotope for each of the three components. For uranium, they would usually be $238-\mathrm{U} / 233-\mathrm{U}$.

Note that the ratio of the atom percent of the spike isotope in the tracer to that of the same isotope in the sample occurs in the equation. Atom fractions may also be used, but do not mix atom percents and atom fractions--you will be in error by a factor of 100 !

Because the units of the atomic weights will cancel, you can use whatever units you please. Far and away the most convenient are atomic masses; i.e., 238.05 is the atomic mass of 238-U.

\subsubsection{Example of the isotope dilution equation}

Let's try an example. Suppose you are performing an isotope dilution analysis and have the following information:

$\begin{array}{lll} & \text { Sample } & \text { Spike } \\ \text { weight } & 29.6 \mathrm{~g} & 15.5 \mathrm{ug} \\ \text { atomic weight } & 237.98 & 233.04 \\ 233 & 0.0001 & 99.1212 \\ 234 & 0.0055 & 0.0011 \\ 235 & 0.7205 & 0.0022 \\ 236 & 0.0000 & 0.0002 \\ 238 & 99.2739 & 0.8753\end{array}$

Thus, in the equation, $a_{t k}=99.1212$ and $a_{\text {sk }}=0.0001$. The unspiked uranium sample is natural, and there is of course no $233-\mathrm{U}$ in it. Its abundance is set to 0.0001 (1 ppm) to avoid dividing by zero, a task at which the computer rebels and kicks you out of the program. Assigning a small value to this variable causes no algebraic problems since it appears both in the ratio $a_{t k} / a_{s k}$ and in $R_{t}$

The ratios can be calculated:

$$
\begin{aligned}
& R_{t}=0.8753 / 99.1212=0.00831 \\
& R_{s}=99.2739 / 0.0001=992.739
\end{aligned}
$$


Let us suppose that we did a particularly good job of spiking and got a $238-\mathrm{U} / 233-\mathrm{U}$ ratio for the mixture of 0.9997 . The equation would now look like this:

$$
C=(15.5 / 29.6)(237.98 / 233.04)(99.1212 / 0.0001) \frac{(0.9997-0.00831)}{(992739-0.9997)}
$$

or

$$
C=(0.5236)(1.0212)(991212)(9.9864 \mathrm{E}-7)
$$

or

$$
\mathrm{C}=0.529 \mu \mathrm{g} / \mathrm{g} \text {. }
$$

\subsubsection{Reverse isotope dilution}

Reverse isotope dilution is a special case of isotope dilution. It is most often encountered when determining the concentration of a spike. When reverse isotope dilution is called for, the variables designated by subscripts $\mathbf{s}$ and $t$ in the equations above are interchanged. This has the effect of making the unspiked sample the spike and the spike the sample.

This option is provided merely as a convenience for the analyst; there is no necessity ever to employ the reverse isotope dilution feature. To use it saves manual entry of information into the Spike and Out files. For a normal isotope dilution analysis, the program assumes information for the spike is in the Spike file and information for the unspiked sample is in the Out file. When a spike is being calibrated, its isotopic and other information is in the Spike file, but, for reverse isotope dilution calculations, it is actually the sample. Similarly, the material most often used to calibrate uranium spikes is NIST U-950 (natural); its isotopic composition is kept in the permanent companion portion of the Out file. To circumvent the need to enter the spike being calibrated in the Out file and NIST U-950 in the Spike file, the concept of reverse isotope dilution was introduced.

To use reverse isotope dilution, you must enter 1 for that index in the set-up part of MSR (item 16--See Section 4.1.16). Enter the file record number in the Spike file occupied by the spike being calibrated. Enter the six-character code that specifies NIST U-950 in the Out file; in the past, this has been NBS950. Enter the weights as though it was a conventional isotope dilution analysis. Nothing more needs to be done. Using the value of 1 for the reverse isotope dilution index as its cue, the program interchanges sample and spike parameters. Isotope dilution calculations proceed as normal. Output is in the usual format.

\subsubsection{Isotope Dilution with No Unspiked Companion}

The Hintenberger isotope dilution equation given in Section 11.1.1 is perfectly general and is applicable to any element regardless of the isotopic compositions involved. Isotope dilution calculations often require two analyses; first for the isotopic composition of the sample, and second for analysis of the sample-spike mixture. The first analysis can be omitted in two different situations. The first is if the isotopic composition of the sample is known; the requisite information need only be entered in the Out file. The second situation for which isotopic analysis of the sample can be omitted is if the spike isotope doesn't occur in the sample. This condition obtains for very few elements, but two of them are uranium and 
thorium, so we perform a lot of analyses in this manner. Our usual uranium spike is 233 , which doesn't occur in nature and is only rarely present in our samples. Similarly, our thorium spike is 230 , which also doesn't occur in nature and which has never been present in our samples.

\subsubsection{Information Entered in MSR}

Enter the Spike file record number and other spike information just as you would for a conventional isotope dilution analysis. The computer is told that you want an isotope dilution analysis without a sample isotopic composition available by entering NO for the unspiked companion. This is all that is required at run time, but is is essential that you have the Ratio file record set up properly, as described in the next section.

\subsubsection{Setting up the Ratio File}

The only difference between the Ratio file record controlling the calculations under consideration and a conventional isotope dilution analysis is the use of the sum variables. The first sum must contain all isotopes of the mixture of spike and sample; the second sum must contain the isotopes of only the unspiked sample. There must be at least one isotope in $\operatorname{sum}(1)$ that isn't in $\operatorname{sum}(2)$, and it must be the spike isotope.

This is all pretty simple in practice. Here's a table for uranium and thorium.

Table 11.1 Sums for Uranium and Thorium

Element

Sum1

Sum2

$$
\frac{\mathrm{U}}{233+234+235+236+238}
$$$$
234+235+236+238
$$

\section{Th \\ $\frac{1}{230+232}$}

232

\subsection{Internal Calibration}

Internal calibration is a way to improve precision and accuracy. A spike with two enriched isotopes is used; the ratio of these two isotopes in the spike should be known as accurately as possible. The value of the ratio between them in a mixture of spike and sample is measured. Comparing the measured value to the known, a bias correction is calculated for each run that applies specifically to the run in question. Improvements of factors of five or more are usually achieved using this technique. Normal analyses use what might be called an average bias correction that is based on the history of the multiplier in use. Routine analysis of isotopic standards assures that the bias correction remains approximately correct. The problem is that an average analysis is like an average person: It's almost impossible to find one. Ionization efficiency is dependelit on a large number of factors, only some of which are known and only a few of which can be controlled. To take one example, it is known that each face of a rhenium crystal has a different work function. The crystallites of the surface of a heated rhenium filament are about the same size as a resin bead. Ionization efficiency is thus dependent on which rhenium crystal face the bead is in contact with. Since there is 
no way of telling which crystal face is exposed, there is no way to load the bead on the face of choice.

From the analyst's point of view, an internal calibration analysis is no more difficult than a conventional isotope dilution analysis. This is only at run time, though. A lot of work must be done before internal calibration analyses can be performed. Most of this is involved with measuring the isotopic composition of the two-isotope spike. It is crucial to the success of the operation that the ratio of the two spike isotopes be known to the best possible accuracy and precision. If the spike is to be used to refine abundance measurements as well as to determine concentration, the abundance of the affected isotopes must also be very well known.

Let's take uranium as an example. The two-isotope spike is made of enriched 233-U and $236-U$ in a ratio that is near 1.0. 236-U is not only expensive, it is in extremely short supply. The higher the 236 assay, the more expensive and rare it becomes. For that reason, two uranium internal calibration spikes were made. ${ }^{11,12}$ The first of these, dubbed the high purity spike, used a $236-\mathrm{U}$ component that was $99.674 \%$ pure. The second spike (low purity) used $236-\mathrm{U}$ that was $89.27 \%$ pure. The latter spike had a $235-\mathrm{U}$ abundance of $9.25 \%$. One of the goals of the use of such calibration techniques was to improve the accuracy of $235-\mathrm{U}$ measurements. A few experiments showed that this could not be done using the low purity spike; the $235-\mathrm{U}$ in the spike was too large to be corrected with sufficient precision and accuracy.

The other element for which an internal calibration spike was made was plutonium. 242-Pu and 244-Pu were the spike isotopes. The situation for 244-Pu is similar to that for 236-U: It is rare and exceedingly expensive.

To determine the internal calibration ratio in the double spike, its isotopic composition is first measured as though it were a normal analysis. Armed with this information as a first approximation, an internal calibration analysis is performed using a suitable certified isotopic standard as the internal calibration spike; NIST U-500 is used for uranium and NIST Pu-947 is used for plutonium. The improved isotopic composition resulting from this analysis is then used for the unspiked sample, and another internal calibration analysis is performed as before. This results in a ratio known to high level of precision and accuracy (usually better than $0.1 \%)$.

I will not describe the results of various internal calibration experiments here; they are covered in several publications. ${ }^{11-13}$ The algebra used to solve the simultaneous equations is described separately. ${ }^{14}$ They are given in Section 8.14 of the Technical Manual. The thing to keep in mind is that this technique works very well and should be considered if and when the best precision and accuracy are required.

Internal calibration calculations are implemented by entering appropriate information into the Spike and Ratio file records that hold the relevant information. One of the entries in the Spike file is for the internal calibration ratio; this is 0 for normal isotope dilution calculations and non-zero for internal calibration. 233/236 is the value that should be entered for uranium; $244 / 242$ should be used for plutonium. In the Ratio file, the base mass for bias correction for uranium must be 236 and that for plutonium 242. The calculations will not be 
mathematically exact unless this is done. The reason for this comes through application of the bias correction. If the bias correction is, say, $0.3 \%$ per mass, then using 238 as the base mass, the bias correction for 236 is 0.994 and that for 235 is 0.991 . If 236 is used, the correction for 235 is 0.997 . The discrepancy enters because $0.991 / 0.994$ is equal to 0.99698 and not to 0.99700 .

\subsection{Instrument Calibration Calculations (NIST U-500)}

It is crucial to accurate analyses to have a properly calibrated mass spectrometer. Each analysis has to have the appropriate corrections applied to the calculations. We have: traditionally divided such corrections into those for dead time and those for bias. The dead time is measurable property of the counting system; bias is a general category that includes all other corrections. These parameters are determined by analysis of certified isotopic standards; the only one we have ever used for direct calibration of an instrument is NIST U. 500. Other standards are used to monitor the bias on a routine basis, correcting as seems appropriate. The dead time can be measured with an oscilloscope or through analysis of a standard. We often do both. Dead times usually need checking only when a multiplier is changed; bias corrections should be monitored routinely by keeping control charts (Section 12.2).

\subsubsection{Dead time}

Dead times for our systems typically range from 10 to 20 nanoseconds, which is pretty good but not spectacular. The dead time imposes a counting rate on the system which, if exceeded, will result in an unacceptably high count-loss correction. Keeping our count rates below, say, 700,000 counts per second is all we need to do to address this issue.

\subsubsection{Bias correction}

Contributors to the bias include isotopic fractionation in the evaporation process; isotopic discrimination during extraction in to the source lens; any discrimination arising during transmission through the instrument; and bias in conversion of the ion to secondary electrons at the collector.

The basic assumption of bias correction is that a measured value for a ratio can be correcied to the certified value by application of a multiplicative factor. This relationship can be described by the following equation:

$$
R_{o} b=R_{c}
$$

where $R$ is the calibration ratio ( $235 / 238$ for uranium), $o$ is observed (or measured), $c$ is certified, and $\mathrm{b}$ is the overall bias. Performing a little freshman algebra, we get:

$$
b=R_{c} / R_{o} \text {. }
$$


Application of this equation assumes that bias correction varies linearly with mass-hence the concept of bias correction per mass. This assumption is not strictly valid--in fact, it just isn't true. When confined to a small enough mass range, however, deviations from linearity are lost in the uncertainty of the measurement. It is thus a perfectly legitimate approximation when applied over the range spanned by the isotopes of a single element. It is not valid, however, to apply a bias correction determined for uranium to a region far removed from it in mass. Even lead (204-208) is too far away.

Note that bias corrections, at least in theory, should always reduce the number of counts in the lighter isotope with respect to those in the heavier. This is because the dominant contributor to bias for most elements is unequal extraction from the region of the filament in the changing extraction field, with lighter ions being extracted more efficiently. Because we sweep the voltage, the extraction field experienced by each isotope as it is being monitored is slightly different from all the others. This in turn imposes a bias on the measurements. For uranium, for example, the bias correction applied to 235 should always be less than 1.000 when compared to that applied to 238 .

Recent experience with iron, nickel, and chromium has shown that elemental factors can also be significant. This is why one should determine the bias correction explicitly for each element. Determining bias correction becomes problematic when there are no certified isotopic standards available. All one can do is to use the natural element and assume that its composition matches that reported by IUPAC. ${ }^{8}$ This works well enough in most instances; if greater accuracy is mandatory, the parties involved in the experiment must agree that a given sample of the element in question has a specified composition. It is then used as the calibration reference in all laboratories. If later the reference values are found to be in error, it is relatively simple to apply the necessary corrections.

\subsubsection{How the program works}

NIST U-500 will be used to illustrate the workings of the program. This is the only standard we have ever used for instrumental calibration, so it seems reasonable to ignore other possibilities. There is no reason in principle, however, why another standard couldn't be used.

\subsubsection{Calculation of bias}

The ratio of 235 to 238 in NIST U-500 is 0.9997 . This is sufficiently close to 1.06000 to assume that any deviations from it are due to bias and not to dead time; the count rates of the two isotopes will be so close to equal that count loss corrections will also be equal. The measured value of the ratio is compared to the certified and the bias correction required to correct the measured value calculated. The following equation is used.

$$
\text { bcpm }=\left(1.0-R_{c} / R_{o}\right) /\left(m_{2}-m_{1}\right)
$$


where:

$$
\begin{array}{ll}
\text { bcpm } & =\text { bias correction per mass } \\
\mathrm{R} & =235-\mathrm{U} / 238-\mathrm{U} \\
\mathrm{c} & =\text { certified } \\
\mathrm{o} & =\text { observed (i. e., measured) } \\
\mathrm{m} & =\text { isotopic masses }
\end{array}
$$

For NIST U-500, $R_{c}$ is $0.9997, m_{2}$ is 238 , and $m_{1}$ is 235 . If $R_{o}$ is 1.006 , the equation gives:

$$
\text { bcpm }=(1.0-0.9997 / 1.006) / 3=0.0021
$$

or $0.21 \%$ per mass.

\subsubsection{Calculation of dead time}

Once the bias is corrected, the program moves on to calculating the dead time. In NIST U-500, the ratio used for this calculation is 234/235. This ratio is certified to be 0.01043 . The measured ratio is corrected for bias; the deviation of the measured value from the certified is ascribed to counting loss on the 235 peak. The equation used to calculate dead time is as follows.

$$
T=\left(R_{c}-R_{o}\right) /\left(C^{*}(t-1.0)\right)
$$

where:

$$
\begin{aligned}
& T=\text { new value of dead time } \\
& R=\text { ratio }(234 / 235) \text { corrected for bias for } R_{o} \\
& C=\text { count rate of } 234 \text { corrected for bias } \\
& c=\text { certified } \\
& O=\text { observed (measured) } \\
& t=\text { estimated dead time }
\end{aligned}
$$

Note that the equation requires an estimate of the dead time before it can be applied. This estimate must be reasonably close for the result to be valid. The program uses the previous value of the dead time for this estimate.

\subsection{Use of Batch Codes}

The batch code was conceived with two purposes in mind. One was that it would serve as a convenient means of identifying all samples from a given project; we used PN as the batch code for pine needles, for example. The second use for the batch code is to use it to designate samples that require special treatment of some kind. Table 11.1 lists the batch codes that have special meaning; a more detailed description follows the table. 


\section{Table 11.2 Batch Codes}

\begin{tabular}{ll} 
Code & \multicolumn{1}{c}{ Description } \\
AQ & Suppress extra Pu bias correction \\
A1 & A126 sample \\
BU & Burn-up analysis \\
M\& & MIST sample \\
RC & Fractionation study \\
R., R/ & RIMS study \\
[] & Bayne statistical study
\end{tabular}

\subsubsection{Batch code AQ}

Plutonium run from a resin bead requires more bias correction than when run as a solution. The program therefore has to know in which form the sample is loaded. Because most of our samples are run from resin beads, I chose to do this by making those run as solutions the special case. The batch code $A Q$, in conjunction with the element symbol PU, tells the program to apply the bias correction for solution loadings.

The way the program works is to have the extra bias required for resin-bead loaded samples stored as bias(1) in the Dtbias file. All plutonium samples except those with batch code AQ will have this extra bias added to that stored as bias(4); those with batch code AQ will not have the extra bias added. See Section 3.3 for a description of the Dtbias file.

\subsubsection{Batch code A1}

This code was originally used to designate samples associated with ISPO project A126. This was a large collaborative program administered by the International Atomic Energy Agency; results had to be reported in a specific format different from ours. A separate file for results was created to facilitate this operation.

Any sample with a batch code of A1 will have its results stored in file A126.DAT as well as in Out.DAT. This is a convenient method of preserving results indefinitely since records in A126 are not written over while those in Out are written over every 3,000 samples.

\subsubsection{Batch code BU}

$\mathrm{BU}$ is the batch code used to identify reactor burn-up analyses. Neodymium is used as a measure of how long a reactor has been operating and at what levels of power--its burnup. Setting up the files for these calculations and the calculations themselves are described in Section 11.5. 


\subsubsection{Other batch codes}

The other batch codes listed in Table 11.1 were used for special research projects and should be of no concern to the analyst beyond the caution not to use them--you may get unexpected results!

\subsection{Burn-up Calculations}

One of the parameters of great interest to engineers is the amount of burn-up a reactor has undergone. Measuring burn-up requires a complex and very expensive analysis. ASTM procedure E321 is the one used here. ${ }^{15}$

To perform the calculations, knowledge of the isotopic composition of uranium and plutonium in the spent fuel rod is required, as is the total amount of each element. Neodymium forms as a fission product in reactor operation, and its isotopic composition and concentration are used, through a complex series of equations, to help calculate burn-up. Thus, one burn-up analysis requires isotope dilution analyses of three elements: $N d, U$, and $\mathrm{Pu}$. Each isotope dilution analysis requires analysis of both unspiked and spiked samples, resulting in a total of six measurements of isotopic composition. Because the samples are highly radioactive, much of the sample preparation must be done in a hot cell. It is easy to see why burn-up analyses are very expensive.

I see no need to go through the algebra in detail here; it is covered in the ASTM manual. It is important, however, that you understand how to arrange for successful calculations.

The ASTM procedure uses the 148-Nd isotope as the basis for the calculations; some engineers prefer to use the sum of $145-\mathrm{Nd}$ and $146-\mathrm{Nd}$, feeling this yields more reliable results. Both systems have been implemented in the program.

The analytical problem is exacerbated by the fact that cerium is also a fission product, and its contribution to the neodymium spectrum at mass 142 must be removed before the calculations can proceed. Neodymium ionizes more readily than cerium; corrections for cerium are therefore applied to neodymium after analysis of cerium. A method similar to that used for uranium-plutonium pairs run trom resia beads (Section 11.6) is used to effect this correction. The six-character sample identification code for the neodymium is entered as the resin bead companion for cerium; no resin bead companion is required for neodymium. It is also necessary to have the batch code of the unspiked cerium sample set to BU. At the end of data-processing for the cerium sample, the neodymium composition is corrected for cerium and the results written to the neodymium record in the Out file. This corrected composition is thus that of the unspiked neodymium sample.

Correction for $\mathrm{Ce}$ is only necessary in the unspiked sample to help establish the amount of $\mathrm{Nd}$ present. The 142 isotope is not used in burn-up calculations, so it is unnecessary to correct the 142 in the spiked sample. 
The spiked neodymium sample is analyzed. Our spike is $150-\mathrm{Nd}: 148-\mathrm{Nd}$ is used as the sample isotope for isotope dilution calculations. The spiked $\mathrm{Nd}$ sample requires an unspiked companion, entered just as it would be for a conventional isotope dilution analysis. To access burn-up calculations, the batch code must be set to BU.

How to set up the calculations is summarized in Table 11.3

Table 11.3 Setting up Burn-Up Calculations

\begin{tabular}{|c|c|c|c|}
\hline Element & Type & Batch $\underline{\mathrm{RB} \text { companion }}$ & Spike companion \\
\hline $\mathrm{Nd}$ & Unspiked & & \\
\hline $\mathrm{Ce}$ & Unspiked & $\mathrm{BU}$ & Nd unspiked \\
\hline $\mathrm{Nd}$ & Spiked & BU & Nd unspiked \\
\hline
\end{tabular}

Note that the unspiked neodymium is used as a companion both for the unspiked cerium and for the spiked neodymium analyses. In the former case, the Out file will hold the neodymium composition uncorrected for cerium; in the lar $r$, it will be corrected.

\subsection{Mixed Uranium-Plutonium Analysis from Resin Beads}

An essential component of nuclear safeguards programs is the assay of fissionable material in a given controlled area. This requires measuring the isotopic composition and concentration of uranium and plutonium. These elements are difficult to separate chemically, and they occur together in spent reactor fuel. The first step in fuel reprocessing is to dissolve the spent fuel rods. The resulting solutions are highly radioactive and present an extremely hostile environment for the analyst. By proper adjustment of nitric acid strength, the two elements of interest can be absorbed together on anion resin beads. It is thus desirable to analyze both elements from the same bead, and we developed a procedure to do so. ${ }^{16}$

The analytical situation would be straightforward if it weren't for the fact each element has a 238 isotope. In spent reactor fuel, $238-\mathrm{Pu}$ is usually a minor component, while $238-\mathrm{U}$ is the major isotope of uranium. Plutonium, with an ionization potential of about $5.7 \mathrm{eV}$, ionizes 100-200 degrees lower than uranium (I.P. $=6.2 \mathrm{eV}$ ). Plutonium is thus analyzed first; difficulty arises because there is usually about 100 times more uranium than plutonium in the dissolver solution. It is often difficult to keep the $238-\mathrm{U}^{+}$intensity low enough to allow a good 238-Pu analysis.

The situation with uranium is better; plutonium is burned off the filament before uranium analysis begins, but, even if this is less than complete, the contribution of 238-Pu to the uranium spectrum is usually small.

\subsubsection{Analyzing resin bead samples}

In the analysis, plutonium is analyzed first, keeping the intensity of 239-Pu, the major plutonium isotope, at about 100,000 counts per second. This is usually low enough to keep 
uranium ionization below acceptable limits. $235-\mathrm{U}$ is monitored and used to effect a correction to the 238 mass position. This is a disadvantageous analytical position: $235-\mathrm{U}$ is usually only about $1 \%$ of $238-U$. The number of counts collected for $235-\mathrm{U}$ must therefore be multiplied by 100 or more to determine the contribution of uranium to the 2.38 mass position. It is usually not possible to obtain a really good 238-Pu measurement from mixed resin beads; if it is important to do so, the two elements should be chemically separated before loading into the mass spectrometer. Fortunately, safeguards doesn't require the best possible analysis for 238-Pu. The most important plutonium isotopes for them are 239 and 240 , the two most abundant.

Once sufficient data have been accumulated for plutonium, that element is burned off by raising the temperature of the filament. The 239-Pu signal is monitored to determine when it is safe to start the uranium analysis. A 1:1 ratio between 239-Pu and 238-U is usually good enough, although it is better if the 239 is smaller than that. 239-Pu is monitored during the uranium analysis to provide a measure of the correction needed to the 238 position. Unlike the case for uranium in plutonium, this is a pretty good correction. 239-Pu is usually the most abundant $\mathrm{Pu}$ isotope, and the correction to 238 is usually quite small.

\subsubsection{Calculating results}

A two-pass algorithm is used to process data from plutonium-uranium pairs. The data are processed for each element as though each had been analyzed alone; on the second pass, corrections based on the results of the first pass are applied to the data.

All this is invisible to the analyst. To signal for mixed bead calculations, all you do is enter the appropriate six-character sample identification code for the variable called "Resin bead companion." Note tha: the companion for plutonium will be the uranium sample and vice versa. Once set up in this way, the programs do all the rest, producing reduced result sheets for the first pass and complete ones for the second. The number of counts subtracted from the 238 mass position is listed in the heading material. Each sample of the pair is inserted twice in the calculational queue, as illustrated in Table 11.4.

Table 11.4 Calculational Sequence for Mixed Resin Beads

$\begin{array}{lll}\frac{\text { No. }}{1 .} & \text { Original } & \frac{\text { Final }}{\mathrm{U}} \\ 2 . & \mathrm{U}^{*} & \mathrm{Pu}^{*} \\ 3 . & \mathrm{Pu}^{*} & \mathrm{U}^{*} \\ 4 . & \mathrm{U}^{*} & \mathrm{Pu}^{*} \\ 5 . & \mathrm{Pu} & \mathrm{U}^{*} \\ 6 . & & \mathrm{Pu}\end{array}$

Sample numbers 2 and 3 in the table form a mixed pair and are marked by asterisks. The computer determines this and inserts each sample twice in the queue so that each will be processed twice before the original samp'e 4 is reached. 
A common source of confusion arises when both members of mixed resin bead pairs need to have their data reprocessed. To make the second pass work properly, the number of raw counts in the Sumcts file is adjusted for the correction applied to the 238 mass position. This means that, if you call for a two-pass calculation when reprocessing, the original number of counts for 238 will be wrong. For valid reprocessing of your data for both members of the pair, the Samp file records must be reinitialized for each of them. See Section 3.9 for a description of how to do this.

\subsection{A Word on Statistics}

In setting up sweet factors in the Scan file, it is desirable to keep the goal of the analysis in mind. The precision of a measurement can be no better than that represented by the square root of the number of counts (you can do far worse!). To have a chance at a precision of $1 \%$, you have to collect at least 10,000 counts for the smaller peak in the ratio; if you want $0.1 \%$, it has to be at least $1,000,000$. Doubling a sweep factor for a minor peak should improve its statistics by a factor of 1.4. This is why we have traditionally used sweep factors that are powers of two--improvements of less than a factor of 1.4 didn't seem worth the effort. Four or eight is the largest sweep factor recommended for most analyses. There are exceptions, as always.

\subsubsection{Measures of Precision}

Standard deviation is the most common measure of precision that we use. It is defined as:

$$
s d=\frac{{\sqrt{\sum\left(x_{i}-x\right)}}^{2}}{(n-1)}
$$

where $\mathrm{x}$ is the average of $\mathrm{n}$ individual measurements whose values are $\mathrm{x}_{\mathrm{i}}$. The standard error of the mean is then $s d / \sqrt{ }$. The $95 \%$ confidence interval (the range you are $95 \%$ sure to hit if running a replicate analysis) is the standard error of the mean times a value from a table well-known to statisticians but not so well known to analytical chemists.

The standard deviation and the $95 \%$ connfidence interval are numerically quite similar, so it doesn't matter a whole lot which is reported. Remember that one, or at most two, significant figures are all that should be reported. A standard deviation of 0.3217 is really no different than 0.3 .

Relative standard deviations and standard errors of the mean are obtained by dividing the deviation or error by the measured value. They are usually reported as percents. 


\section{QUALITY ASSURANCE AND CONTROL}

Quality assurance and control are crucial elements in any successful laboratory operation. They are essential in the measurement of isotopic ratios, so much so that quality control programs have been in place in our laboratory for more than thirty years.

\subsection{Availability of standards}

Whenever possible, instrument performance should be tied to analysis of certified isotopic standards. These have traditionally been available from the National Bureau of Standards (NBS), which is now called the National Institute of Standards and Testing (NIST). New Brunswick Laboratory (NBL) has in recent years assumed the responsibility of distributing radioactive isotopic standards. The European equivalent of NBL, the Central Bureau for Nuclear Measurements (CBNM), also sells isotopic standards.

Availability of standards is spotty. Driven by the needs of the nuclear community, there is an extensive suite of uranium standards that range from $0.2 \% 235-\mathrm{U}$ to $97 \% 235$. $\mathrm{U}$. There are three plutonium standards. Aside from these, standards are available on a hit or miss basis. Those of interest to geologists are pretty well represented: boron, strontium, and lead all are available. One of the biggest gaps is the rare earth region; not one rare earth element has an isotopic standard available. Most of the elements we analyze for the Stable Isotopes program have no standards.

Dead time is an instrumental constant and will be the same for all elements. The value determined using an NIST U-500 should apply to all elements. The same is most emphatically not true with regard to bias correction. Bias correction varies not only with mass but also with the chemical nature of the element. When an appropriate certified isotopic standard is available, it should be used to measure bias. When a standard is not available, our only recourse is to define one. Spex Industries sells a kit of almost all solid elements. Using the element of interest from this or some similar source, bias is measured on the assumption that the element's isotopic composition is natural; i.e., its composition is that recommended by the International Union of Pure and Applied Chemistry (IUPAC) in their compendium of atomic weights. ${ }^{8}$ Many elements are known to vary in isotopic composition in nature, but most have reasonably well-defined "normal" values. In any case, we can do no better.

To determine the bias correction for some element without a standard, analyze the natural element (the one defined as the reference) in replicate enough times to achieve statistically reliable results. Normally at least three replicates wili be required, but there will be times when more are needed and occasions when two are enough; the number of replicates depends on the desired accuracy to which you need to measure the element in question.

\subsection{Analyzing standards}

There are two distinctly different types of analysis performed for quality assurance and control purposes. One is to determine instrumental calibration parameters, and the other is to monitor these values on a routine basis. 


\subsubsection{Instrumental calibration}

The standard used for instrumental calibration has always been NIS'T U-500. Details of its use are given in Section 11.3. This standard has a composition designed for such use: the 235-U/238-U ratio is almost exactly 1.00 and is thus ideal for measuring bias correction independent of dead time. The 234-U/235-U ratio is used to measure dead time. When analyzing NIST U-500 samples, it is important to have a healthy count rate to assure statistical reliability of the dead time measurement; $234-\mathrm{U}$ is about $1 \%$ of 235 , and the sweep factor for the 234 mass position should be four or eight to improve its statistics. The count rate should be at least 500,000 counts per second for either 235 or 238 .

\subsubsection{Routine standards}

It is important to run a standard often enough to verify proper instrument operation. This is rather vague; the frequency at which such standards should be analyzed will vary with conditions. For most cases, running one standard per five or six samples is a good rule to follow. There are times of stable multiplier operation and heavy sample load when one standard every two days suffices. For example, if you are running a large number of uranium samples (say 25 a week) and the multiplier has shown no hint of instability, running one standard for every ten samples is sufficient.

The routine standard should, if at all possible, be the element under analysis. It does no good to run a uranium standard when you are analyzing lead. If no certified standard is available, use the reference sample of the element; its selection is described above.

\subsection{Control charts and their interpretation}

A very useful way to track an instrument's performance is through use of a control chart. I will use uranium as an example because that is the element with which we are most familiar, but control charts can be generated for any element analyzed often enough to provide statistical reliability.

\subsubsection{What to plot}

The control charts in popular use for such purposes are called Shewhart charts by the statisticians. These charts plot a variable as a function of time; through them sinister trends can be identified and, one hopes, addressed. Since we deal with isotopic ratios, that is what we plot. It is important to use a scale with sufficient resolution that significant deviations from acceptable performance are readily identified and yet not so fine that normal statistical spread is exaggerated to the point of confusing presentation of the information.

For uranium, we plot the measured value of $235-\mathrm{U} / 2.38-\mathrm{U}$ for each standard analyzed. 234-U/238-U and 236-U/238-U are also plotted, but they are not as important as the 235/238. For each ratio, the certified value of the ratio is indicated to allow easy identification of the scatter about it. It is desirable to indicate various deviations from the expected value; $1 \%$ and $2 \%$ for the $235 / 238$ ratio are convenient. The values of the relevant ratios are plotted using some convenient scale for time along the $X$ axis of the plot. This need not be linear: it is only important to spot trends, and quantifying them serves no useful purpose. 


\subsubsection{Interpreting control charts}

The most important aspect of a control chart is that it provides an immediate visual evaluation of performance. A single point, no matter how far from the accepted value, has little meaning; about all one can do with a one-point anomaly is to see what the next analysis brings. Any trend, however, is potentially significant. If a number (say 5 or more) of consecutive 235/238 ratios all fall on one side of the accepted value line, it is a signal to keep a close eye on things. If the trend continues, a new bias correction per mass should be calculated and inserted in the Dtbias file. Note that this is the responsibility of the Group Leader, but the analyst should alert him or her of any troubling trends.

While the $235 / 238$ is the most important diagnostic ratio, the others are useful, too. Because these isotopes are present at $50-70 \mathrm{ppm}$, the precision of their ratios will not be as good as it is for 235. A standard deviation of $2 \mathrm{ppm}$ or less is normal. As an example of using these ratios, suppose both the $234 / 238$ and $236 / 2.38$ ratios are consistently low. The setting of the background subgroup should be checked. It is possible it is picking up $\mathrm{Th}^{+}$at the 232 mass position, and too large a background correction is being applied.

Another instance allowed identification of a sweeping problem. It was observed that 236 was always high, and always high by about the same amount, implying some systematic error. Even though this bias was only a few ppm, the matter clearly warranted investigation. A bit of detective work revealed that, on the downward sweep across the mass spectrum, a few counts of 238 spilled over into the 236 mass position, giving rise to the observed discrepancy. The problem was corrected by scanning the 237 mass position; the unwanted 238 counts then went into the 237 position, which has no analytical utility. The reason why 238 spilled over in the first place (the root cause!), however, has not been uncovered.

Quality control documents not only serve a vital function in operation of the laboratory, but also help during audits. Every auditor I have encountered has agreed that they are the best way to maintain control over instrumental performance. I encourage all analysts to be conscientious in maintaining the charts. 


\section{APPENDIX: EXAMPLES OF INPUT}

This appendix contains examples of commonly required input. The aim here is to provide a quick one-page reference for the most frequently encountered analyses. Input for the Samp, Spike, and Ratio files is listed as needed. The first example describes information needed for all samples, and that needed for various special cases follows. Although the examples have been chosen to represent realistic cases, the records chosen in any given illustration may not be appropriate. You still need to know what you are doing.

\subsection{Input required for all samples}

Set-Up Window for MSR

1. SAMP file record no.

2. SCAN file record no.

3. Sample id

4. Descriptive title

5. Batch Code

6. RATIO file record no.

7. Resin bead companion id.

8. SPIKE file record no.

9. Unspiked companion id.

10. Isotope dilution ratio

11. Spike weight

12. Sample weight

13. Dilution weight

14. Aliquot weight

15. Density

16. Reverse iso diln index
12

$1 \mathrm{El} \mathrm{U}$

U10039

Isotopic analysis

11

0

0

1.0

1.0

1.0

1.0

1.0

Index no. to correct, 0 to proceed:

Note that the minimum information required is the Samp file record number (assigned by the computer), a Scan file record number, the six-character sample identification code, and the Ratio record number. The descriptive title has been included because it's a good idea to use it, but it isn't mandatory. There follows an example of the Ratio file. 
Contents of Ratio File

1. Element symbol

2. Base mass for bias correction

3. Normalizing mass for atom pcts

4. Mass to be subtracted from SGs

5. Sum No. 1

6. Sum No. 2

7. Sum No. 3

8. Difference No. 1

9. Difference No. 2

10. Difference No. 3

Ratios 111-11

11. $233 / 238$

12. $234 / 238$

13. $235 / 238$

14. $236 / 238$

15. 315.238

16. $238 / 233$

17.

18.

19.

20.

21.
$\mathrm{U}$

Record no. 11 238

238

315

$233+234+235+236+238$

No entry

No entry

No entry

No entry

No entry

Ratios 12-22

22.

23.

24.

25.

26.

27.

28.

29.

30.

31.

32. 
13.2 Isotope dilution analysis

Set-Up Window

Input parameters

1. SAMP file record no.

13

2. SCAN file record no.

3. Sample id.

2 E1 U

U10040

4. Descriptive title

Iso Diln

5. Batch Code

6. RATIO file record no.

7. Resin bead companion id.

8. SPIKE file record no.

9. Unspiked companion id.

10. Isotope dilution ratio

$0 \quad 0$

11. Spike weight

0.771

1.006

13. Dilution weight

1.0

14. Aliquot weight

1.0

15. Density

1.0

16. Reverse iso diln index companion id

17. Contam el symbols

18. Blank

Index no. to correct, 0 to proceed

Spike File

1. Element symbol

U Record No. 5

2. Label for spike

3. Atomic weight

New low level 233 spk, 11-9-83

4. Internal calibration index

233.0411

5. Isotope dilution ratio

0

$238 / 233$

6. Internal calibration ratio

0 Mass

Abundance

7. 233

0.9996860

8. 234

0.0000030

9. 235

0.0000050

10. 236

0.0000001

11. 238

0.0003060 
13.3 Isotope dilution analysis with no companion

\subsubsection{Uranium analysis}

Set-Up Window

Input Parameters

1. SAMP file record no.

14

2. SCAN file record no.

3. Sample id.

$1 \quad \mathrm{E} 1 \quad \mathrm{U}$

4. Descriptive title

U10041

5. Batch Code

6. RATIO file record no.

No companion

7. Resin bead companion id.

8. SPIKE file record no.

9. Unspiked companion id.

10. Isotope dilution ratio

11. Spike weight

1

12. Sample weight

13. Dilution weight

14. Aliquot weight

15. Density

16. Reverse iso diln index 17. Contam el symbols

18. Blank companion id.

Index no. to correct, 0 to proceed:

Contents of Ratio File

1. Element symbol U

2. Base mass for bias correction

3. Normalizing mass for atom pcts

4. Mass to be subtracted from all SGs

5. Sum No. 1

6. Sum No. 2

7. Sum No. 3

8. Difference Nos. $1,2,3(8,9,10)$ Ratios 1-11

11. $2133 / 238$

12. $234 / 238$

13. $235 / 238$

14. $236 / 238$

15. $234 / 18$

16. $235 / 18$

17. $236 / 18$

18. $238 / 18$

19. $238 / 18$

20.

21.
Record no. 14

238

238

315

$233+234+235+236+238$

$234+235+236+238$

No entry

No entry

Ratios 12-22

22.

23.

24.

25.

26.

27.

28.

29.

30

31.

32.

Note Sum(2). 
13.3.2 Thorium analysis

$$
\text { Set-Up Window }
$$

Input Parameters

1. SAMP File record no.

15

2. SCAN file record no.

3. Sample id.

4. Descriptive tiels

15 E1 TH

T10042

5. Batch code

6. RATIO file record no.

7. Resin bead companion id.

8. SPIKE file record no.

9. Unspiked companion id.

10. Isotope dilution ratio

11. Spike weight

Thorium with no companion

12. Sample weight

13. Dilution weight

101

14. Aliquot weight

15. Density

16. Reverse iso diln index

17. Contam el symbols

23

NO

$0 \quad 0$

0.554

3.3321

1.0

1.0

1.0

Index no. to correct, 0 to proceed:

Contents of Ratio File

1. Element symbol TH

Record no. 23

2. Base mass for bias correction

232

3. Normalizing mass for atom pcts 232

4. Mass to be subtracted from all SGs

475

5. Sum No. 1

6. Sum No. 2

$230+232$

7. Sum No. 3

232

8. Difference No. 1

No entry

9. Difference No. 2

No entry

10. Difference No. 3

No entry

Ratios 1-11

No entry

11. $230 / 232$

Ratios $12-22$

12. $232 / 230$

22.

23.

13. $475 / 232$

24.

14.

25.

15.

26.

16.

27.

17.

28.

18.

29.

19.

30.

20.

31.

21.

32.

18. Blank companion id. 
13.4 Internal calibration

$$
\text { Set-Up Window }
$$

Input Parameters

1. SAMP file record no.

16

2. SCAN file record no.

3. Sample id.

$1 \mathrm{E} 1 \quad \mathrm{U}$

4. Descriptive title

U10043

5. Batch code

6. RATIO file record no.

Internal calibration

7. Resin bead companion id.

8. SPIKE file record no.

9. Unspiked companion id.

10. Isotope dilution ratio

11. Spike weight

12. Sample weight

13. Dilution weight

14. Aliquot weight

15. Density

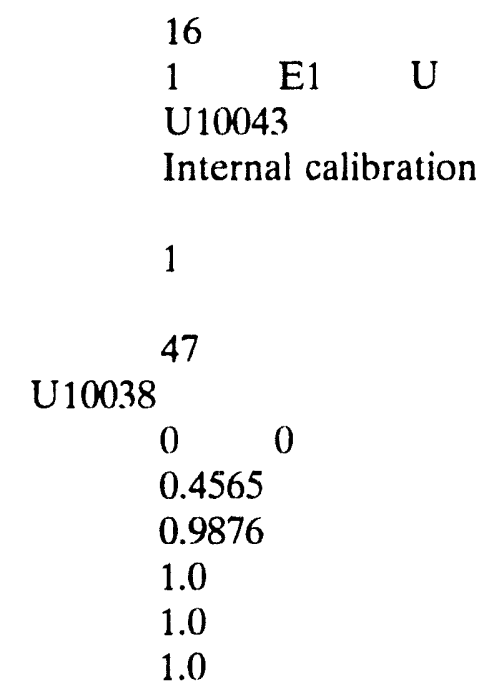

16. Reverse iso diln index. 17. Contam el symbols

18. Blank companion id.

Index no. to correct, 0 to proceed:

Spike File

1. Element symbol

2. Label for spike

3. Atomic weight

4. Internal calibration index

5. Isotope dilution ratio

6. Internal calibration ratio Mass

7. 233

8. 234

9. 235

10. 236

11. 238
U Record no. 5

High purity int std, 1-82

2.34 .6350

0

$236 / 238$

233/236

Abundance

0.4693520

0.0001550

0.0010840

0.5286390

0.0007710 
13.5 Mixed uranium-plutonium pairs

Set-Up Window for Plutonium

Input Parameters

1. SAMP file record no.

17

2. SCAN file record no.

3. Sample id.

$15 \quad \mathrm{E} 1 \quad \mathrm{Pu}$

4. Descriptive title

P10044

5. Batch code

6. RATIO file record no.

7. Resin bead companion id.

8. SPIKE file record no.

9. Unspiked companion id.

10. Isotope dilution ratio

11. Spike weight

Pu companion

12. Sample weight

41

U10045

0

13. Dilution weight

14. Aliquot weight $\quad 1.0$

15. Density

1.0

16. Reverse iso diln index 17. Contam el symbols

18. Blank companion id.

Index no. to correct, 0 to proceed:

Set-Up Window for Uranium

Input Parameters

1. SAMP file record no.

17

2. SCAN file record no.

3. Sample id.

$0 \quad 0$

1.0

1.0

1.0

4. Descriptive title

P10045

Pu companion

5. Batch code

6. RATIO file record no.

1

7. Resin bead companion id.

U10044

8. SPIKE file record no.

0

9. Unspiked companion id.

10. Isotope dilution ratio

11. Spike weight

$0 \quad 0$

12. Sample weight

1.0

13. Dilution weight

1.0

14. Aliquot weight

1.0

15. Density

1.0

1.0

16. Reverse iso diln index. 17. Contam el symbols

18. Blank companion id.

Index no. to correct, 0 to proceed: 
13.6 Neodymium Burn-Up Analysis

Set-Up Window for Neodymium

Input Parameters

1. SAMP file record no.

20

2. SCAN file record no.

3. Sample id.

4. Descriptive title

N10048

5. Batch code

$\mathrm{Nd}$ for burn-up

6. RATIO file record no.

BU

71

7. Resin bead company id.

N10047

8. SPIKE file record no.

37

9. Unspiked companion id.

10. Isotope dilution ratio

11. Spike weight

$0 \quad 0$

12. Sample weight

1.073

1.029

13. Dilution weight 1.0

14. Aliquot weight $\quad 1.0$

15. Density

1.0

16. Reverse iso diln index 17. Contam el symbols

18. Blank companion id.

Index no. to correct, 0 to proceed:

Set-Up Window for Cerium

Input Parameters

1. SAMP file record no.

20

2. SCAN file record no.

3. Sample id.

$56 \quad \mathrm{E} 1 \quad \mathrm{Ce}$

4. Descriptive title

5. Batch code

C10048

6. RATIO file reco:d no.

7. Resin bead companion id.

Ce for burn-up

$\mathrm{BU}$

71

8. SPIKE file record no.

N10047

0

9. Unspiked companion id.

10. Isotope dilution ratio

$0 \quad 0$

11. Spike weight

1.0

12. Sample weight

1.0

13. Dilution weight

1.0

14. Aliquot weight

1.0

15. Density

1.0

16. Reverse iso diln index 17. Contam el symbols

18. Blank companion id.

Index no. to correct, 0 to proceed. 
13.7 NIST U-500

\author{
Set-Up Window
}

Input Parameters

1. SAMP file record no

2. SCAN file record no.

3. Sample id.

4. Descriptive title

5. Batch code

6. RATIO file record no.

7. Resin bead companion id.

8. SPIKE file record no.

9. Unspiked companion id.

10. Isotope dilution ratio

11. Spike weight

12. Sample weight

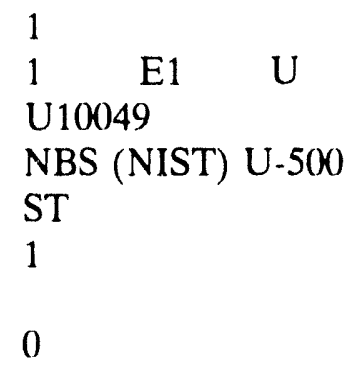

1

$1 \quad \mathrm{E} 1 \quad \mathrm{U}$

U10049

NBS (NIST) U-500

ST

1

0

$0 \quad 0$

1.0

1.0

13. Dilution weight $\quad 1.0$

14. Aliquot weight $\quad 1.0$

15. Density

1.0

16. Reverse iso diln index 17. Contam el symbols

19. Record number in STAND file 0

18. Blank companion id. Use 0 for dead time and bias calc. Index no. to correct, 0 to proceed:

Note appearance of index 19. 
13.8 NIST U-010

\section{Set-Up Window}

Input Parameters

1. SAMP file record no.

2. SC.AN file record no.

3. Sample id.

2
$2 \quad$ E1
U10050
NBS (NIST)

ST

5. Batch code

6. RATIO file record no.

7. Resin bead companion id.

8. SPIKE file record no.

9. Unspiked companion id.

10. Isotope dilution ratio

11. Spike weight

12. Sample weight

13. Dilution weight

14. Aliquot weight

15. Density

16. Reverse iso diln index

19. Record number in STAND file Use 0 for dead time and bias calc. Index no. to correct, 0 to proceed:
1

0

$0 \quad 0$

1.0

1.0

1.0

1.0

1.0

18. Blank companion id. 


\section{ACKNOWLEDGMENTS}

The programs in this manual would not be in their present condition without input from a number of individuals. First and foremost, Ray Walker and his analytical problems dictated the presence of many of the options in $M S C$. He also checked the validity of many of the calculations. Edd Miller made numerous suggestions helpful in improving the "userfriendliness" of the programs. Charlie Pritchard, John Sites, and Ray Eby all contributed helpful requests that made the programs more versatile. W. H. Christie, who wrote the Section's first versions of the program, was always ready with advice and answers. Last, but not least, Doris Smith typed the manuscript, deciphering my copper-plate handwriting and coping patiently with the multitude of underscores and mysterious variable names. Our thanks to all of them. 


\section{REFERENCES}

1. D. H. Smith and H. S. McKown, "New FORTRAN Computer Programs to Acquire and Process Isotopic Mass Spectrometric Data," ORNL/TM- (91 or 92)

2. D. H. Smith, W. H. Christie, H. S. McKown, G. R. Hertel, Int. J. Mass Spectrom. and Ion Phys. $\underline{10}, 343$ (1972-73).

3. A. E. Cameron, W. H. Christie, H. S. McKown, W. T. Rainey, and D. H. Smith, USAEC Report ORNL-464.3, January 1971.

4. D. H. Smith, "New FORTRAN Programs to Acquire and Process Isotopic Mass Spectrometric Data," ORNL/TM-77002, September 1979

5. D. H. Smith, "New FORTRAN Computer Programs to Acquire and Process Isotopic Mass Spectrometric Data," ORNL/TM-8356, August 1982

6. D. H. Smith, ed., ORNL/TM-6485, November 1978.

7. W. H. Christie and H S. McKown, Chem. Instrum 3, 99 (1971).

8. P. D. Bievre and P. D. P. Taylor, Int. J. Mass Spectrom Ion Proc. $\underline{123}, 149$ (1993).

9. D. H. Smith, H. S. Mckown, W. H. Christie, R. L. Walker, and J. A. Carter, ORNL/TM-5485, June 1976.

10. H. Hintenberger in "Electromagnetically Enriched Isotopes and Mass Spectrometry," M. L. Smith, ed., Academic Press, New York, NY, 1958, pp. 177-189.

11. D. H. Smith and R. L. Walker, Institute of Nuclear Materials Management 13(2), 44 (1985).

12. D. H. Smith, R. L. Walker, and J. A. Carter, USDOE Report ORNL/TM-9774, Sept. 1985.

13. D. H. Smith, R. L. Walker, and J. A. Carter, USDOE Report ORNL/TM-9820, Nov. 1985.

14. D. H. Smith, ORNL/TM-9842, Nov. 1985.

15. D. H. Smith, ASTM Annual Book of Standards, Part 45: Nuclear Standards, American Society for Testing and Materials, Philadelphia, PA, 1981, pp. 978-987.

16. D. H. Smith, R. L. Walker, J. A. Carter, "Resin Bead Procedure for U, Pu," Anal. Chem. $54,827 \mathrm{~A}$ 


\section{DISTRIBUTION}

1. C. M. Barshick

2. R. M. Coleman

3. D. C. Duckworth

4. E. H. McBay

5-9. H. S. Mckown

10. S. A. McLuckey

11. R. L. McPherson

12. L. R. Riciputi

13. W. D. Shults

14-18. D. H. Smith

19. M. L. Turner

20. R. E. Valiga

21. Patent Section

22. Laboratory Records

23. Laboratory Records-RC

24. Central Research Library

25. Document Reference Section

26-27. Office of Scientific and Technical Information, Oak Ridge, TN 37830

28. Assistant Manager of Energy Research and Development, Oak Ridge, TN 37830 

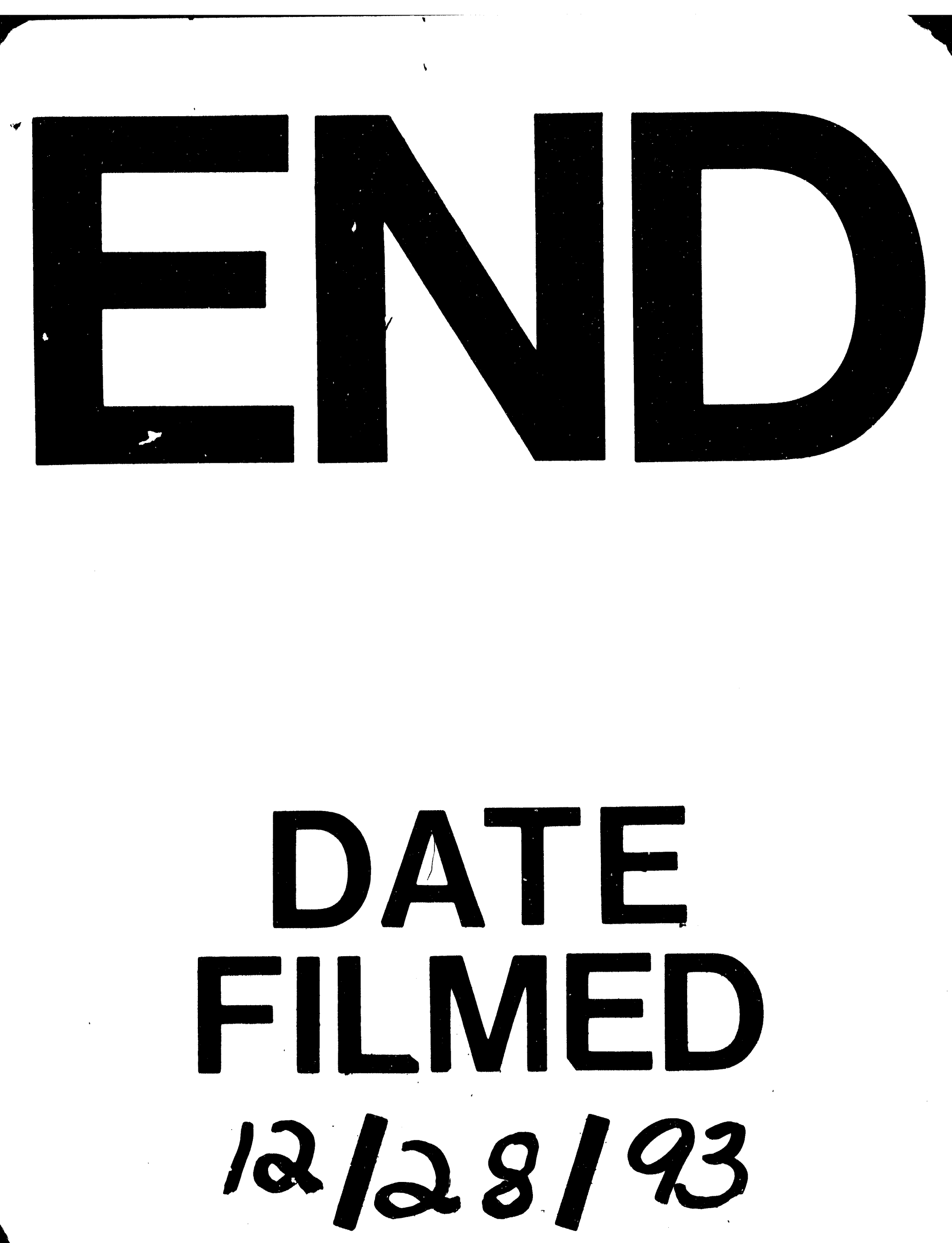

1 
